NASACONTRACTOR REPOR T

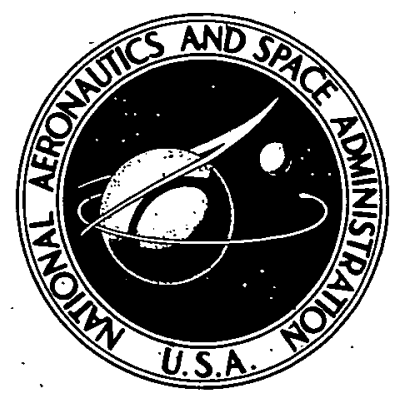

NASA CR-2466

0
$\vdots$
$\vdots$
$\approx$
$\Sigma$

\title{
DIGITAL ADAPTIVE FLIGHT CONTROLLER DEVELOPMENT
}

by Howard Kaufman, Gurbux Alag, Paul Berry, and Samir Kotob

Prepared by

RENSSELAER POLYTECHNIC INSTITUTE

Troy, N.Y.

for Langley Research Center

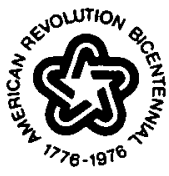

national aeronautics and SpaCe administration - WaShington, D. C. • DECEMber 1974 


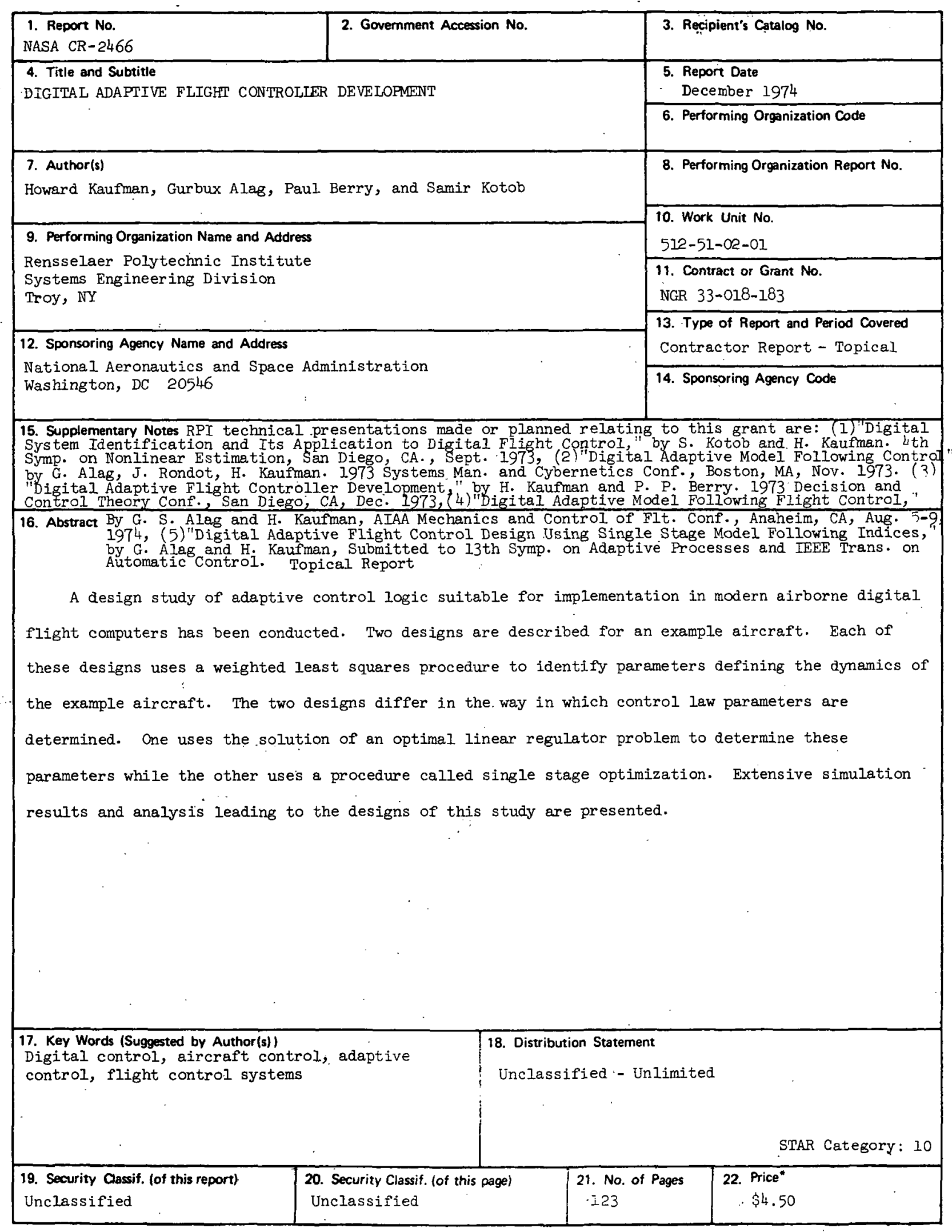

"For sale by the National Technical Information Service, Springfield, Virginia 22151 


\section{FOREWORD}

The investigation described in this report was performed by the Systems Engineering Division of Rensselaer Polytechnic Institute for the Flight Dynamics and Control Division of the Langley Research Center as a part of the Digital Fly-By-Wire Program. It was carried out during the period September 15, 1972 - October 15, 1973. The investigation was headed by Professor Howard Kaufman who was assisted by three graduate students. 


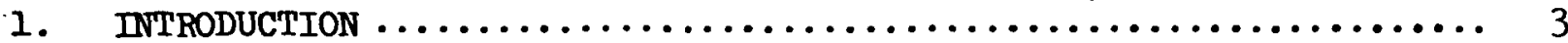

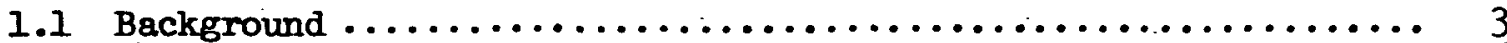

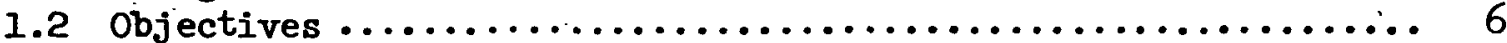

1.3 Scope and outline $\ldots \ldots \ldots \ldots \ldots \ldots \ldots \ldots \ldots \ldots \ldots \ldots \ldots \ldots \ldots \ldots$

1.4 Significance $\ldots \ldots \ldots \ldots \ldots \ldots \ldots \ldots \ldots \ldots \ldots \ldots \ldots \ldots \ldots \ldots \ldots \ldots$

2. REQUIREMENTS FOR DIGITAL ADAPTIVE FLIGHT CONTROL . ............. 10

2.1 System Representation ........................... 10

2.2 Performance Evaluation $\ldots \ldots \ldots \ldots \ldots \ldots \ldots \ldots \ldots \ldots \ldots \ldots \ldots \ldots \ldots$

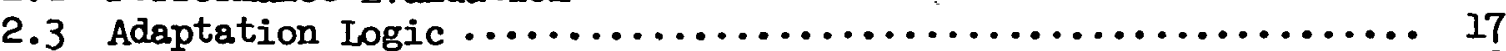

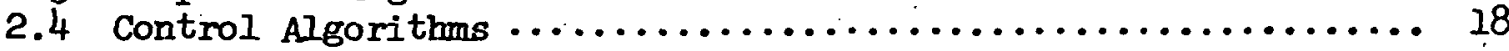

2.4 .1 Perfect Model Following ..................... 18

2.4 .2 Single stage optimization $\ldots \ldots \ldots \ldots \ldots \ldots \ldots \ldots \ldots \ldots \ldots \ldots . \ldots \ldots$

2.4.3 Infinite Time Optimal quadratic Control ............ 24

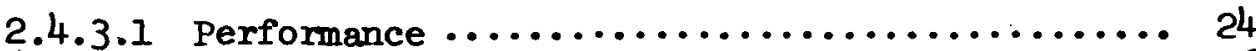

2.4.3.2 Controller structure .................. 26

2.4.3.3 Infinite Time I.inear Optimal Regulator

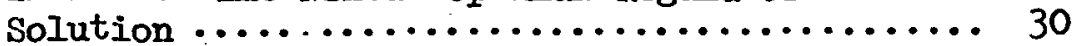

2.5 Parameter Identification $\ldots \ldots \ldots \ldots \ldots \ldots \ldots \ldots \ldots \ldots \ldots \ldots \ldots \ldots \ldots$

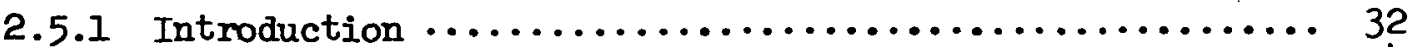

2.5.2 Problem statement ........................... 34

2.5.3 Procedures for Parameter Estimation only ........... 35

2.5.3.1 Weighted Least Squares Parameter

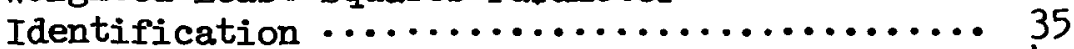

2.5.3.2 Stochastic Gradient Estimation ............ 41

2.5.4 Procedures for Simultaneous. State and Parameter

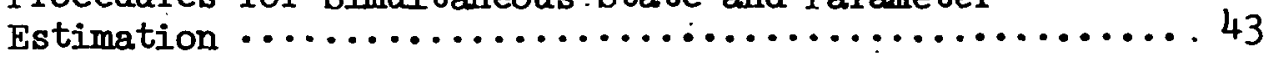

2.5.4.1 Fxtended Kalman Filter ................. 43

2.5.4.2 Separate Linear state and Parameter

Estimation (SLSPE) ................... 50

3. DIGITAL ADAPTIVE CONTROLLER REQUIREMENTS .................. 53

3.I Considerations Concerning the Adaptive Algorithms ......... 53

3.2 Adaptive Logic Based Upon Single Stage Optimization

Procedures ................................... 56

3.3 Adaptive Logic Based on the Linear Optimal Regulator

Solution $\ldots \ldots \ldots \ldots \ldots \ldots \ldots \ldots \ldots \ldots \ldots \ldots \ldots \ldots \ldots \ldots \ldots \ldots \ldots$

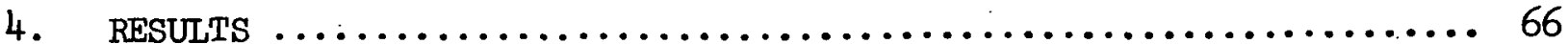

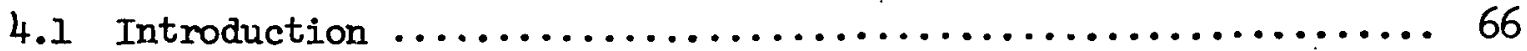

4.2 State Sensitivity of Parameter Estimation Errors ..........66 
4.2 .1 General Procedure ......................... 68

4.2.2 Sensitivity Results for Single stage optimization...... 70

4.2.3 Sensitivity Results for the Linear Optimal Regulator... 71

4.3 Identification Results....................... 72

4.3.1 Results Pertinent to procedures for Parameter

Estimation only ......................... 73

4.3.2 Results Pertinent to Estimation of Both States

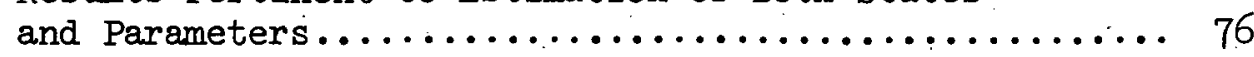

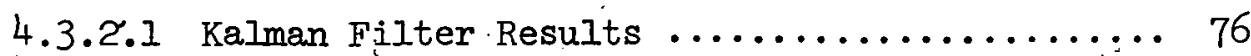

4.3.2.2 SISPE Results ................... 79

4.4 Performance Under Perfect Identification Prior to

Interfacing Adaptive Control Logic ................. 79

4.4 .1 Performance of the Single Stage Algorithm........... 79

4.4.2 Performance of the Linear Optimal Regulator........... 82

4.5 Adaptive Control System Performance Evaluation............ 92

4.5.1 Adaptive Control Loop Experiment - Single Stage

Optimization Procedure....................... 92

4.5.2 Adaptive Control Loop Experiment - Linear Optimal

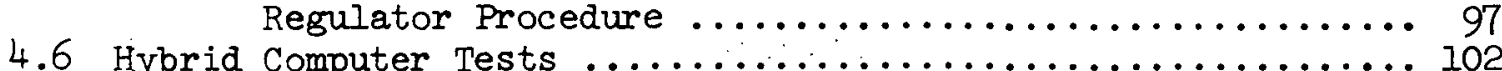

4.6 .1 Linear Simulation Results.................... 102

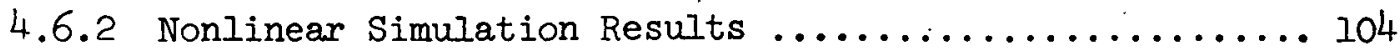

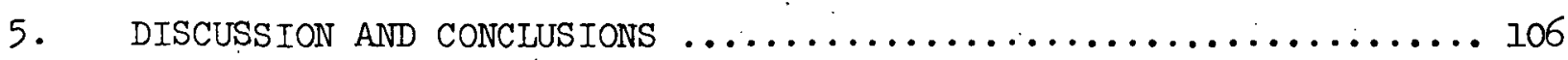

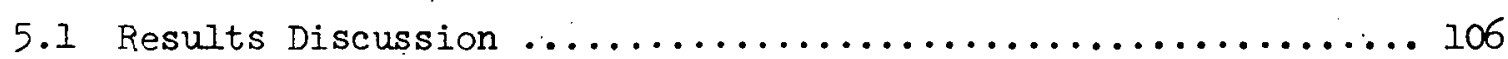

5.2 Feasibility of Implementation $\ldots \ldots \ldots \ldots \ldots \ldots \ldots \ldots \ldots \ldots \ldots \ldots$

5.3 Recommendations for Future Research ................. 108 
List of Symbols

$A_{m}$

AN

A

$\mathrm{B}_{\mathrm{m}}$

$\mathrm{B}_{\mathrm{N}}$

$\mathrm{B}_{\mathrm{p}}$

e

$F, F_{p}$

$f_{i}, f_{c}, f_{g}$

FC

G, $G_{p}$

$\mathrm{H}$

$I\left(I_{p}\right)$

$\mathrm{J}$

$\mathrm{K}$

$\mathrm{K}_{\mathrm{m}}$

$\mathrm{K}_{\mathrm{p}}$

$\mathrm{K}_{\mathrm{p}}$

$\mathrm{K}_{\mathrm{x}}$

$\mathrm{K}_{21}, \mathrm{~K}_{22}, \mathrm{~K}_{23}, \mathrm{~K}_{24}$

$l_{i}$

lTOT
Model system matrix

Nominal system matrix

Plant system matrix

Model input matrix

Nominal input matrix

Plant input matrix

Error vector, $x_{m}-x_{p}$

Continuous system matrices

Frequencies for identification, control, and gain update respectively

Flight Condition

Continuous input matrices

Measurement selector matrix

Identity matrix (of dimension $p \times p$ )

Performance index

Feedback or Kalman gain matrix

Gain matrix multiplying pilot input $u_{m}$

Gain matrix multiplying plant input $u_{p}$

Gain matrix multiplying plant state $x_{p}$

Gain matrix multiplying model state $x_{m}$

Gain matrices as defined in fig. 7, multiplying respectively $x_{p}, u_{p}, x_{m}, u_{m}$

Total number of parameters to be identified from $i^{\text {th }}$ row of $A_{p}$ and $i^{\text {th }}$ row of $B_{p}$

Total number of parameters to be identified 


\section{$\mathbf{n}$}

m

$p$

p

Q

q

$\hat{q}$

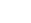

$\hat{q}_{0}$

$R_{i}(k)$

$\mathbf{r}$

$\mathbf{s}$

$T_{c}, T_{g}, T_{i}$

$u_{m}$

$u_{p}$

$\mathbf{v}$

$v_{p}$

$x_{a}$

x m

II

$x_{p}$

$\hat{x}_{p}$

$\mathrm{y}_{\mathrm{p}}$

z

$\alpha$
Number of plant or model states

Number of inputs

Riccati gain matrix or covariance of estimate Incremental roll rate

Weighting matrix or fictitious noise covariance

Incremental pitch rate or vector of parameters to be identified

Parameter estimates

Initial parameter estimate

Gain matrix for stochastic gradient procedure

Incremental yaw rate

Vector of parameters not requiring identification

Periods between control updates, gain updates, and identification respectively

Pilot input vector

Aircraft input vector

Incremental velocity

Per sámple change in the aircraft control $u_{p}$ Accumulated error between plant and model states

Model state vector

Nominal state vector

Plant state vector

Estimate of $x_{p}$

Aircraft measurement vector

Component conmon to all simgle stage gains, $\mathrm{z}=\left[\begin{array}{lllll}R+\mathrm{B}_{\mathrm{p}}^{T} & Q & \mathrm{~B}_{\mathrm{p}}\end{array}\right]^{-1} \mathrm{~B}_{\mathrm{p}} \quad \mathrm{Q}$ (fig. Il)

Incremental angle of attack 
$\beta$

$\Delta \hat{x}_{p}$

$\delta$.

$\delta$

$\delta_{\mathbf{r}}$

$\delta_{\mathrm{T}}$

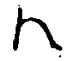

$\theta$

$\xi$

$\sigma_{i}^{2}$
Incremental sideslip angle

Estimate of $x_{p}-x_{N}$

Incremental aileron deflection

Incremental elevator deflection

Incremental rudder deflection

Incremental thrust

Measurement noise vector

Incremental pitch angle

Fictitious parameter noise

Variance of $\xi_{i}$ 
Digital Adaptive Flight

Controller Development

by

Howard Kaufman*, G. Alag**,

P. Berry**, and S. Kotob**

\section{Summary}

Digital fly-by-wire flight control systems are currently of interest because simple mechanical linkages are no longer able to cope with the many control problems associated with high performance aircraft and space vehicles. In view of this, adaptive flight control algorithms have been developed for ultimate implementation and flight testing in an onboard digital computer.

In order to achieve this objective, the following hierarchy of activity was pursued:

- Development of a direct digital controller amenable to on-line adaptation.

- A study of on-line discrete parameter identification procedures.

- A development of digital adaptive flight control logic suitable for inflight implementation.

The above analysis was performed using equations of motion and the related parameters for a typical fighter ai rcraft as supplied by NASA.

As a result of computational experiments performed using typical measurement noise characteristics, two configurations are recomended, namely:

* Assist. Professor of Systems Engineering Division, Rensselaer Polytechnic Institute, Troy, N. Y. 12181

* Graduate students at Rensselaer Polytechnic Institute, Troy, N. Y. 12181 
- An interfacing of linear quadratic optimal regulator logic with a weighted least squares estimator.

- An interfacing of control logic, designed using single stage performance indices, with a weighted least squares estimator. 


\subsection{INTRODUCTION}

\subsection{Background}

Digital fly-by-wire flight control systems are currently of interest because simple mechanical linkages are not able to cope with the many control problems associated with high performance aircraft and space vehicles. 1,2 Digital implementation is extremely advantageous because of:

- The significant weight and volume savings.

- The availability of low cost integrated circuits.

- The ability to design complex controllers which previously were impossible to implement onboard an aircraft.

- The high reliability of digital logic.

- The capability for time sharing multiple control loops.

Furthermore the need for an adaptive control system capable of control adjustment has been established for ${ }^{3,4}$ :

Providing uniform stability and handling qualities over the complete flight envelope despite drastic changes in the open loop characteristics of the aircraft.

Providing acceptable flying qualities over a wide range of external: disturbances due to atmospheric turbuience and outer loop command signals.

Design of a digital adaptive flight control system requires careful. consideration of the following factors:

Computer storage limitations

Computer cycle time Computer operation time

Stability requirements

Parameter changes Control limitations Pilot input signals 
Control hysteresis

Test signal renuirements

Turbulence effects

Development of such a system can be guided by assigning a heirarchy of structure to the system as shown in fig. 1. At the lowest level, directly interacting with the aircraft dynamics, is the Direct Digital Controller (DDC). This consists of a set of gains that multiply the appropriate states along with the necessary summing logic. Because this DDC controller operates directly on the aircraft, it is executed at the fastest rate. Typical sample rates might be 30 times per second.

Because any given set of DDC gains cannot be satisfactory over this complete flight envelope, it is necessary to incorporate some sort of logic that adjusts these gains whenever necessary. Thus the adaptation logic is placed at the second and third control levels.

For small parameter deviations, and subsequently small deviations in performance, an implicit adaptation algorithm might be used. This does not require explicit parameter identification and could be used at a sample rate intermediate to the DDC logic and the explicit adaptation logic.

Finally, in order to adjust the DDC gains in the presence of large parameter variations, some type of on-line identification appears necessary in order to guarantee system stability: The execution rate of this logic is clearly dependent upon the rate of parameter variation and must be determined through experimentation.

A summary of the state of the art in adaptation can be found in ref. 5. Some of the more salient conclusions of this study are:

For nonminimum phase plants, the only methods that can achieve good control are those using some form of 


\section{ADAPTATION CONTROL}
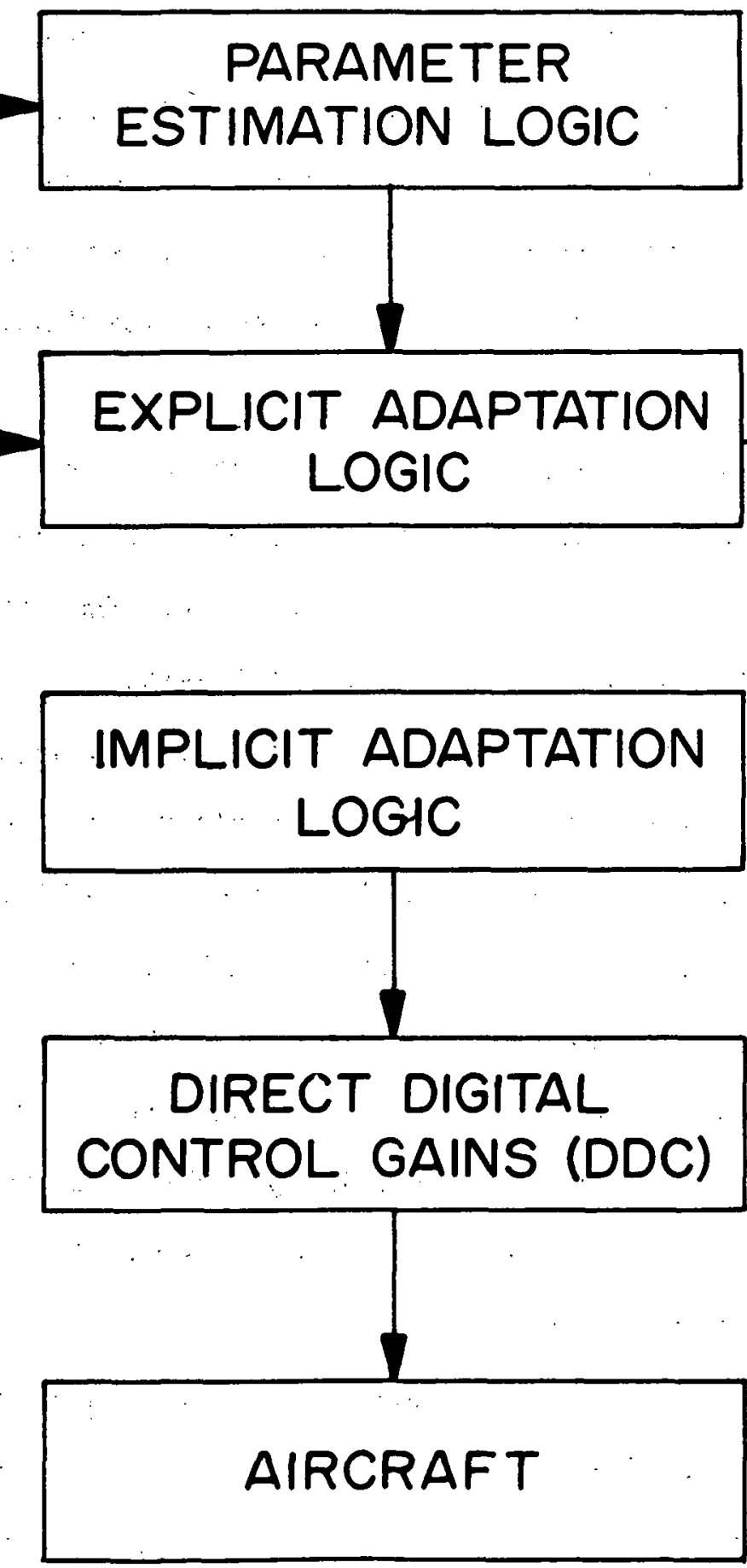

FIG.1 Digital adaptive flight control system. 
explicit parameter identification.

- Adaptive techniques requiring explicit plant identifi-

cation tend to require the most computational capability.

- The best adaptive control over output performance characteristics is potentially provided by those adaptive methods that explicitly identify the plant.

- Excessive bandwidth (gain) is undesirable because of the resulting sensitivity to measurement noise and the danger of exciting high order structural or sensor modes.

- Control methods which explicitly identify the aircraft have the lowest gain level requirements because the gains can be adjusted directly to their proper values which are functions of the airframe parameters.

Despite the previously cited studies, an actual digital implementation of a completely adaptive flight controller has to date not been attempted. This may be attributed to the limitations of the available analog equipment as well as the reluctance to change over to digital control. Consequently, a research effort was initiated by NASA Langley Research Center for the purpose of applying modern digital control theory towards the development of an implementable digital adaptive flight controller.

\subsection{Objectives}

In view of the attractiveness of digital adaptive flight control, an effort to develop such a system has been pursued by the Systems Division of 
Rensselaer Polytechnic Institute (RPI) since September 1972. The objectives of this effort have been:

1) To investigate adaptive control procedures which might be capable of being implemented in a digital flight computer.

2) To test these procedures using linearized lateral and - longitudinal data as supplied by NASA for a typical fighter aircraft operating over a given flight envelope.

3) To validate the design using one of NASA's hybrid simulations.

\subsection{Scope and Outline}

Development of an adaptive control system requires consideration of:

I) The system equations of motion.

2) The selection of an index of performance.

3) The comparison of actual process behavior with the desired or ideal behavior.

4) The parameter gain adjustment procedure used to drive the process more towards the desired behavior.

Whereas in the initial phases of the development, the linearized lateral and longitudinal equations of motion are being considered separately. for control purposes, final results will include the performance resulting from the application of various developed controllers to a nonlinear sixdegree-of-freedom simulation. A description of the linearized equations is contained in Section 2.1.

Because it is convenient to express desired aircraft response to 
pilot commands in terms of a mathematical model, all performance indices considered in the development penalize some measure of the error between the process and model outputs. A detailed outline of such "model following" performance is found in Section 2.2.

Usage of such a model also facilitates the instantaneous comparison of process performance with the desired behavior. This leads to the possibility of implementing either implicit adaptation logic which does not require process parameter identification or explicit adaptation logic which indeed does use online parameter estimates. Following a discussion of these alternatives in section 2.3, various control structures amenable to adaptation are presented in Section 2.4. Procedures for online identification are then described in section 2.5 .

Finally based upon the various subsystem designs given in section 2.0, overall adaptive control logic is presented in Section 3.0, and computational results based on this logic are given in section 4.0 .

Recommendations for implementation and for further studies are found in section 5.0 .

\subsection{Significance}

Development of a digital adaptive flight control system is of significance not only to the particular aircraft considered but also to digital process control in general. Such a development represents an important application of modern digital control theory that is a step towards narrowing of the gap between theory and practice. The proposed logic, while feasible for digital process computers, is clearly impractical for analog implementation. Of immediate significance, however, is the demonstration that digital fly-by-wire technology is capable of providing desirable handling 
qualities over the complete flight envelope of a high performance aircraft despite changes in the open loop characteristics of the airframe. 
2.0 REQUIREMENTS FOR DIGITAL ADAPTIVE FLIGHT CONTROL

Items essential to adaptive control design are individually discussed in the following four sections. System representation is first developed in Section 2.1 followed by a discussion of performance evaluation in section 2.2. Adaptation principles and control structure are presented in sections 2.3 and 2.4 respectively. Finally, methods for online parameter identification are presented in section 2.5:

Results pertinent to the tuning and the performance of these procedures are given in Section 4.

\subsection{System Representation}

The linearized dynamics of the aircraft as supplied by NASA may be represented by the vector state equation

$$
\underline{\dot{x}}=\mathrm{F} \underline{\mathrm{x}}+\mathrm{G} \underline{\mathrm{u}}
$$

where $x$ denotes the state vector

$\underline{u}$ denotes the control vector

and $F$ and $G$ are matrices of the appropriate dimensions.

For linearized lateral notion

$$
\begin{aligned}
\underline{x^{\prime}}=\left(\begin{array}{l}
\mathrm{p} \\
\mathrm{r} \\
\beta \\
\varnothing
\end{array}\right) & =\left(\begin{array}{l}
\text { roli rate } \\
\text { yaw rate } \\
\text { sidesilp angle } \\
\text { roll angle }
\end{array}\right) \\
\text { and } \underline{u}=\left(\begin{array}{c}
\delta_{a} \\
\delta_{r}
\end{array}\right) & =\left(\begin{array}{l}
\text { aileron deflection } \\
\text { rudder deflection }
\end{array}\right)
\end{aligned}
$$


For linearized longitudinal motion

$$
\underline{x}=\left(\begin{array}{l}
q \\
v \\
\alpha \\
\theta
\end{array}\right)=\left(\begin{array}{l}
\text { pitch rate } \\
\text { velocity } \\
\text { angle of attack } \\
\text { pitch angle }
\end{array}\right)
$$

and

$u^{*}=\left(d_{e}^{*}\right)=$ (elevator deflection)

The elements of $F$ and $G$, known to vary with mach number and altitude, were provided for a typical fighter aircraft for the six flight conditions (FC's) shown in the flight envelope of Figure 2. These are provided in Appendix A.

The objective of the research was to find implementable digital algorithms for computing the control signals $\delta_{a}, \delta_{r}$, and $\delta_{e}$ so as to insure uniform and desirable handling quantities for an aircraft flying within the given flight envelope. This was to be performed assuming that during flight the elements of $F$ and $G$ were not readily available (e.g., as scheduled functions of mach number and altitude).

Because of the need to implement the control system in a digital flight computer as shown in Fig. 3, Eqn. 2.1 was transformed into the equivalent discrete form:

$$
\underline{x}(k+1)=A \underline{x}(k)+B \underline{u}(k) .
$$

$$
\text { where } \quad \begin{aligned}
A & =\left(\int_{0}^{e^{F T}} e^{F T} d \tau\right) \\
T & =\text { Sampling Time }
\end{aligned}
$$

and $x(k), u(k)$ denote $x, u$ at time $k \cdot T$. 


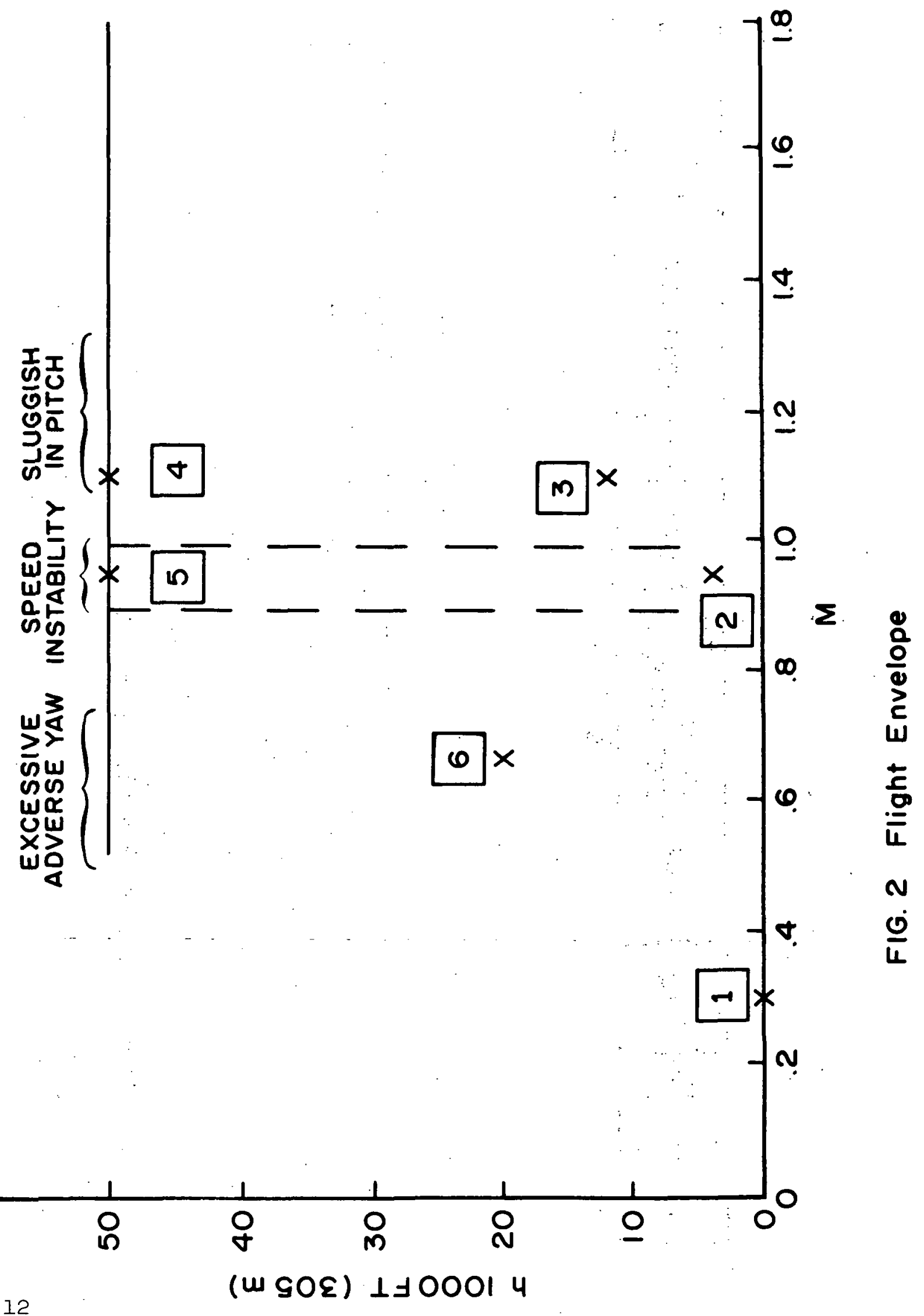


Eqn. 2.2 is a valid representation assuming that $F$ and $G$ do not vary for. $\mathrm{k} T \leqslant \mathrm{t} \leqslant(\mathrm{k}+1) \mathrm{T}$ and that the control signal is constant between sample times, i.e.,

$$
u(t)=u \cdot(k T) \text { for } k T \leq t \leq(k+1) T
$$

This latter relation will be true assuming the use of a zero order hold type of digital to analog converter. The former assumption is valid if the rates of change of $F$ and, $G$ are such that changes over the sample period are negligible.

\subsection{Performance Evaluation}

Inherent to the effectiveness of any adaptive control system is the capability for rapidly assessing the performance and making the necessary modifications to the control gains. One such procedure that fits these requirements and at the same time has the potential for insuring uniform handling qualities is the concept of model following control as depicted in fig. 4. This concept has been of interest to many investigators over the past few years. $6,7,8,9,10$ In fact, relative to these efforts, Erzberger has published a set of "perfect model following" conditions under which the output of the process can be made iaentical to the output of the model.

Being that the ideal objective of model following flight control is to force the aircraft to respond as the model would to a given pilot command,it is often desirable to simulate the online model dynamics in the flight computer and to generate the aircraft control signal using the actual aircraft 


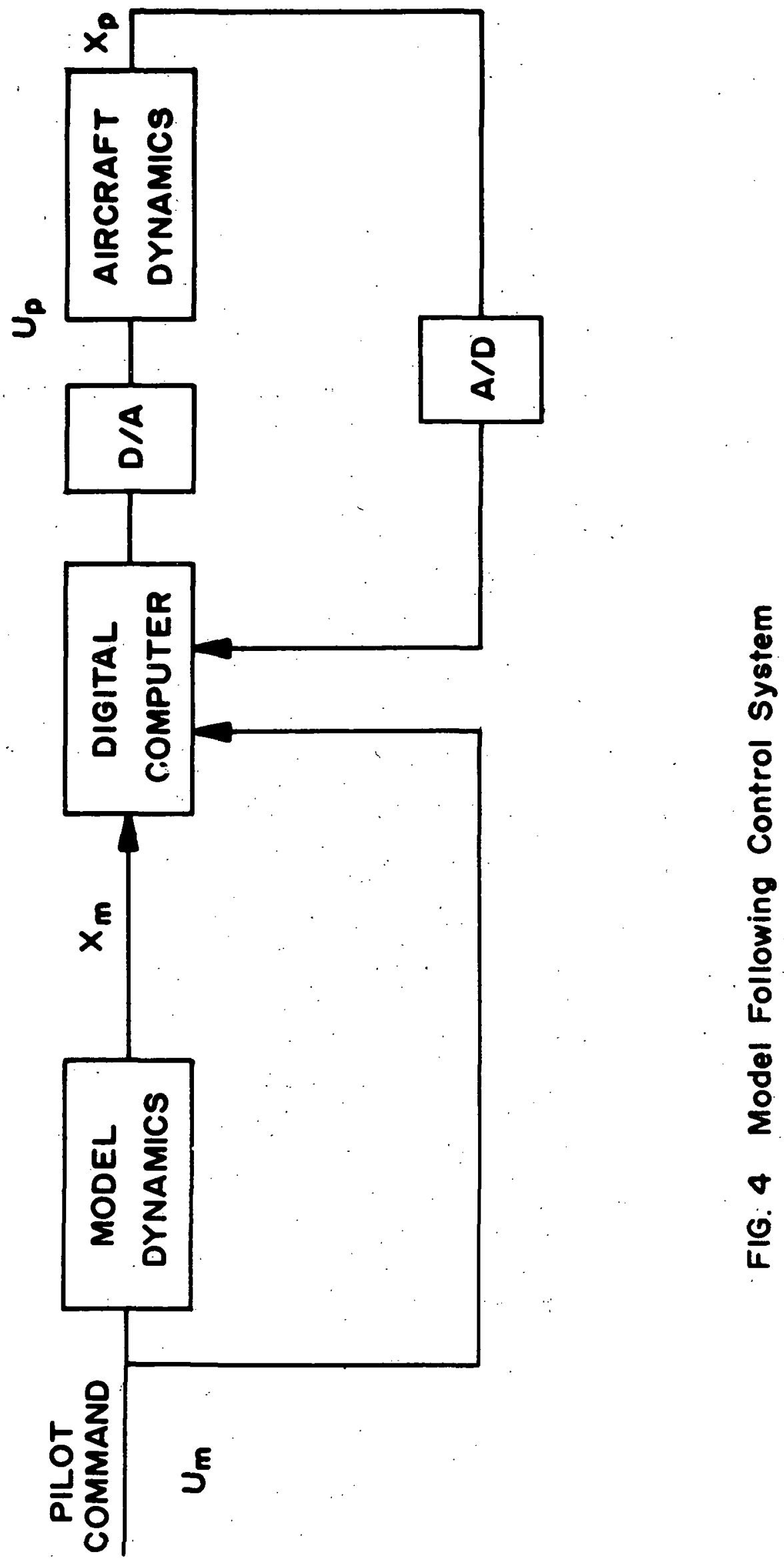

के

옹

$\frac{0}{\frac{0}{3}}$

$\frac{8}{4}$ 
states, the pilot input commands, and the model states. This situation is sometimes referred to as the pilot's flying the computer while the computer. is flying the aircraft.

More precisely, the model following problem can be stated as follows: Given the aircraft dynamics:

$$
x_{p}(k+1)=A_{p} x_{p}(k)+B_{p} u_{p}(k)
$$

where: $\quad x_{p}(k)$ is the aircraft $(n x l)$ state vector at sample time $k$

$$
u_{p}(k) \text { is the (mxl) control vector }
$$

and

$$
A_{p}, B_{p} \text { are matrices with the appropriate dimensions ; }
$$

find the control $u_{p}(k)$ such that the process state vector $x_{p}(k)$ approximates "reasonably well" some model's state vector $x_{m}(k)$ defined by the equation:

$$
x_{m}(k+1)=A_{m} x_{m}(k)+B_{m} u_{m}(k)
$$

where $x_{m}(k)$ is the $(n x l)$ model state vector

$u_{m}(k)$ is the (mxl) pilot input and

$A_{m}, B_{m}$ are matrices of the appropriate dimensions.

One possible variation is to find $u_{p}$ (k) such that the (IxI) dimensional process output vector

$$
H_{p} x_{p}(k)
$$

approximates the model's ( $r x l$ ) output vector

$$
\mathrm{H}_{\mathrm{m}} \mathrm{x}_{\mathrm{m}}(\mathrm{k})
$$


A further possibility to consider is the situation in which there is process measurement noise $\mathrm{n}(\mathrm{k})$ such that the only available process output is:

$$
\mathrm{y}_{\mathrm{p}}(\mathrm{k})=\mathrm{H}_{\mathrm{p}} \mathrm{x}_{\mathrm{p}}(\mathrm{k})+\eta(\mathrm{k})
$$

Turbulence can be considered in the above system by including a process noise term $w_{k}$ such that the plant equation becomes:

$$
x_{p}(k+I)=A_{p} x_{p}(k)+B_{p} u_{p}(k)+\Gamma w_{k}
$$

where

$$
\begin{aligned}
& w_{k} \text { is a stochastic process whose statistics are chosen to } \\
& \text { approximate the atmospheric conditions and } \\
& \Gamma \text { is the corresponding distribution matrix. }
\end{aligned}
$$

\subsection{Adaptation Logic}

In the very early phases of the project, considerable study was given to the tradeoffs involved in selecting either an explicit or an implicit adaptation algorithm, the differences being that

- In explicit adaptation; on-line estimates of the aircraft parameters are used for gain adjustment.

- In implicit adaptation, some measure of the error between the actual and the desired state trajectories is used for gain adjustment. That is, no explicit parameter identification is used.

This study and a similar one made by TASC in $1970^{5}$ indicated that explicit adaptation would initially be preferable because: 
Stability was to be required in the presence of severe parameter variations.

- Effectiveness of implementing implicit adaptation is related to the satisfaction of the conditions of perfect model following. $6,10,11$ These in general will not be satisfied for MASA supplied models.

Implicit adaptive control comparable to the hyperstability approach used by Landau ${ }^{6}$ is to be considered at a later date.

\subsection{Control Algorithms}

\subsubsection{Perfect Model Following}

In a model following (mf) adaptive control system the design goal is to force the compensated system to duplicate the performance of a reference model. The knowledge of plant parameters enables controller gains to be set so as to achieve the desired characteristics.

There are two configurations of $\mathrm{mf}$, known as implicit mf and real $\mathrm{mf}$. As fig. 5 shows, in implicit $\mathrm{mf}$ the aircraft controls are formed from the aircraft states and pilot input. No dynamic coupling exists between the model states and the closed-loop plant; the model state $x_{m}$ appears only in the performance index.

Figure 6 illustrates real model following in which the model states must be generated for use in forming the control law. Alignment of plant and model in the presence of uncertainties such as unknown parameters and random disturbances, requires this type of control. This enables a continuous correction of the errors between model and plant states even in the presence of unknown disturbances. 


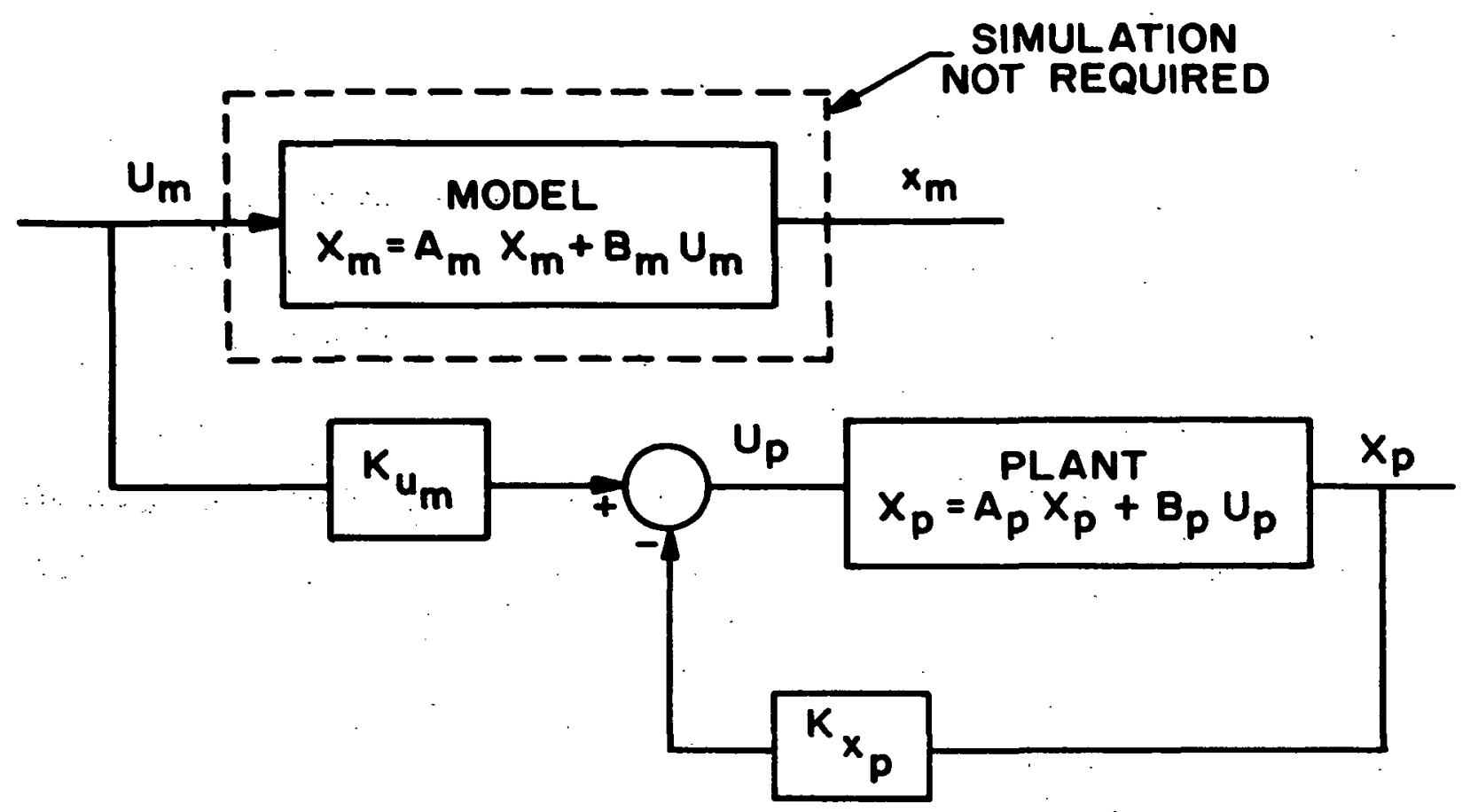

FIG. 5 Implicit Model Following

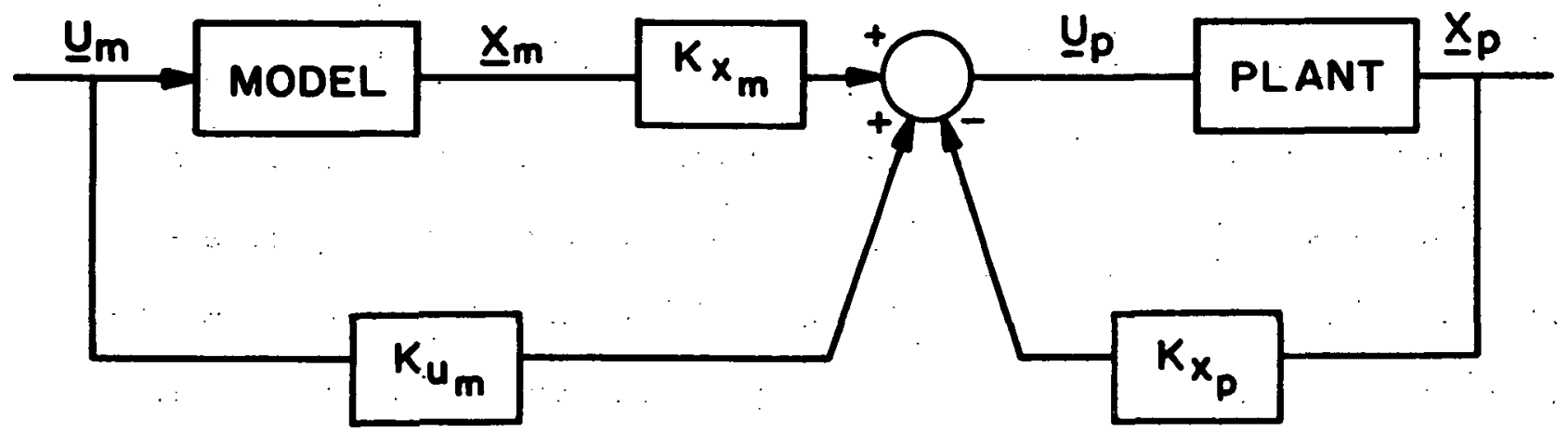

FIG. 6 Real Model Following 
Erzberger has shown the existence of a control for perfect

model following provided that:

$$
\begin{aligned}
& \left(I-B_{p} B_{p}^{+}\right)\left(A_{m}-A_{p}\right)=0 \\
& \left(I-B_{p} B_{p}^{+}\right) B_{m}=0
\end{aligned}
$$

where $\mathrm{B}_{\mathrm{p}}^{+}$is the pseudo inverse of $\mathrm{B}_{\mathrm{p}}$. If these conditions are satisfied, then the control input

$$
u_{p}=B_{p}^{+}\left(A_{m}-A_{p}\right) x_{p}+B_{p}^{+} B_{m} u_{m}
$$

will result in perfect model following.

Chan ${ }^{10}$ has given an alternative control law which yields

perfect following if eqs. 2.7 and 2.8 are satisfied, and which yields

stability in the sense of boundedness otherwise.

This controller has the form:

$$
u_{p}=U_{1}+u_{2}
$$

where

$$
\begin{aligned}
U_{1}= & K_{e} \\
U_{2}= & B_{p}^{+}\left(A_{m}-A_{p}\right) x_{m}+B_{p} B_{m} u_{m} \\
e= & x_{m}-x_{p} \text {, the error vector and } K \text { is a gain matrix chosen } \\
& \text { to stabilize }\left(A_{p}-B_{p} K\right) .
\end{aligned}
$$

Related to the development of perfect model following

controllers is the single stage design approach used by Motyka ${ }^{12}$ for simultaneously weighting both the state error and the control effort at a single time instant. Elimination of the control effort weighting and a slight modification of the error state yields Erzberger's perfect mf control law. 


\subsubsection{Single Stage Optimization}

In view of the work by Chan and Motyka, and the attractiveness of control gains readily adjustable in the presence of parameter changes, single stage indices were considered for digital adaptive control design.

The essential feature of a single stage performance index is the fact that only a single point in time is considered. If $\underline{e}(k)$ describes the error in $\mathrm{mf}$ at sample time $k$, then a performance index of the form $\underline{e}^{T}(k) Q \underline{e}(k)$, where $Q$ is a positive semidefinite weighting matrix, allows the designer to specify the relative importance of mf in different states by trading off the size of $m f$ errors in different states. In addition, the plant control signal can also be weighted in the performance index by including a factor of the form $\underline{u}_{p}^{T}(k) \quad R u_{-p}(k)$, where $u_{p}$ is the control signal and $R$ is a positive semi-definite weighting matrix. Such a factor allows further tradeoff between $\mathrm{mf}$ in the plant states and the magnitude of the control signals required to effect that mf. Thus, a performance index of the form

$$
J(k)=e^{T}(k) Q e(k)+u_{p}^{T}(k) R u_{p}(k)
$$

was considered.

$$
\text { To find the vector } \underline{u}_{p} \text { to minimize } J, \nabla_{\underline{u}} \text { J is formed }
$$

and set to zero. The resulting equation gives the vector $\underline{u}_{-p}$.

The first formulation, called RF for "response feedback", as developed by Motyka ${ }^{12}$ for continuous time systems is now discussed here for discrete time. For known present plant state $x_{p}(k)$, the next state $\underline{x}_{p}(k+I)$ can be required to be that state which would result if $x_{p}(k)$ were used in 2.4 instead of $x_{-m}(k)$ i.e., if the plant had the dynamics of the model. Such a 
desired correspondence would be realized if the plant state at $(k+1)$, i.e., $x_{p}(k+1)$ were equal to:

$$
A_{m} x_{p}(k)+B_{m} u_{m}(k)
$$

Thus the error signal $e$ in eq. 2.13 becomes:

$$
\left(A_{p} x_{p}(k)+B_{p} u_{p}(k)\right)-\left(A_{m} x_{p}(k)+B_{m} u_{m}(k)\right)
$$

$\nabla_{u_{p}} J$ is set equal to zero to obtain:

$$
\begin{aligned}
u_{p}(k)= & {\left[R+B_{p}^{T} Q B_{p}\right]^{-1} B_{p}^{T} Q * } \\
& {\left[\left(A_{m}-A_{p}\right) x_{p}(k)+B_{m} u_{m}(k)\right] } \\
= & K_{x_{p}} x_{p}(k)+K_{u_{m}} u_{m}(k)
\end{aligned}
$$

The features of this controller include the following:

(1) It is closed-1oop containing feedback only from the plant and not from the model. The model matrices are, however, used in forming the controller gains;

(2) Steady state behavior of the plant is not necessarily equal to that of the model since model states are not used for comparison;

(3) Real-time simulation of the model is not necessary.

A second formulation, to be denoted the "same state" algorithm requires that the plant states be exactly equal to the model states at the next sampling instant i.e., it requires that: 


$$
x_{p}(k+1)=x_{m}(k+1)
$$

substituting from (2.3) and (2.4), the error signal

$$
\underline{e}=\left(A_{p} x_{p}(k)+B_{p} u_{p}(k)\right)-\left(A_{m} x_{m}(k)+B_{m} u_{m}(k)\right)
$$

Again setting $\nabla_{\underline{-p}}{ }^{J}=0$ gives:

$$
\begin{aligned}
u_{p}(k)= & {\left[R+B_{p}^{T} Q B_{p}\right]^{-1} B_{p}^{T} Q^{*} } \\
& {\left[A_{m} x_{m}(k)-A_{p} \cdot x_{p}(k)+B_{m} u_{m}(k)\right] }
\end{aligned}
$$

or

$$
u_{p}(k)=K_{x_{m}} x_{m}(k)-K_{x_{p}} x_{p}(k)+K_{u_{m}} u_{m}(k)
$$

This SS controller has the following features:

(1) It is closed-1oop with respect to the plant and explicit with respect to the model;

(2). The factor $z=\left[\begin{array}{lll}R+B_{p}^{T} & Q & B_{p}\end{array}\right]^{-I_{B_{p}}^{T}}$ is common

$\therefore \therefore$ to all three gain matrices.

(3) Because $x_{m}$ is explicitly used, steady state differences between plant and model states might be removed given enough independent controlling elements.

(4) Gains can easily be updated on-line without having to solve any nonlinear algebraic (Riccati) equations.

Because of these attractive characteristics, the same state controlier was chosen for implementation in an adaptive control loop, details of which are presented in Section 3.1. 


\subsubsection{Infinite Time Optimal Quadratic Control}

\subsubsection{Performance}

The performance index which is selected for minimization implies the controller type, the degree of model-following, and the overall control effort expended. A typical performance index might consist of some positive semi-definite function of the model following error $\left(x_{p}(k)-x_{m}(k)\right)$ balanced against a positive definite function of the control $u_{p}(k)$. Inclusion of control rate and/or integrals of the model following error will result in a controller with a dynamic structure, i.e., with integral feedback terms. Asseo ${ }^{7}$ has demonstrated that such dynamic feedback can yield reduced sensitivity to plant parameter variations, and other investigators have examined various specific structures. 13,14,15

In particular, Asseo ${ }^{7}$ considered the control rate $\dot{u}_{p}(t)$ rather than the control itself as the independent variable. This necessitated the treatment of the plant control $u_{p}(t)$ as an additional state with the state equation

$$
\dot{u}_{p}(t)=v_{p}(t)
$$

In the discrete case, the integrator is replaced by an accumulator (i.e., a unit delay with unity feedback), and the corresponding equation becomes:

$$
u_{p}(k+1)=u_{p}(k)+v_{p}(k)
$$

An attractive feature of this formulation is the ability to include in the performance index both positive definite functions of $u_{p}(k)$ (to prevent overly large controls) and of $\mathrm{v}_{\mathrm{p}}(\mathrm{k})$ (to prevent overly large control rates). This control structure was the actual one utilized in the experimental studies. 
A second dynamic feedback structure as suggested by Tiroshi and Elliott ${ }^{13}$ is analogous to the classical proportional-integral feedback structure. By integrating the model-plant error, it is expected that any step change in the model states will result in zero steady-state plant-model error. Again the discrete equivalent of an integrator is an accumulator and the state equations for the "integral" are:

$$
x_{a}(k+1)=x_{a}(k)+C\left(x_{p}(k)-x_{m}(k)\right)
$$

The matrix $C$ specifies which model-following errors will be accumulated. Care mast be taken in the selection of the matrix $c$, for an uncontrollable system can result. A description of this difficulty and a test for system controllability is given by Porter. 14 This controller would be especially suitable for a transport type aircraft (because of the accurate steady-state control) but may be more responsive to noisy feedback than the first structure because the proportional-integral controller does include direct feedback of the plant states themselves.

The various possibilities suggested above were broken into two classes of performance indices. The performance index corresponding to the first controller irscludes terms weighting both modelfollowing error and control rate, and has the form;

$$
\frac{1}{2} \sum_{k=0}^{\infty}\left(x_{p}(k)-x_{m}(k)\right)^{T} Q\left(x_{p}(k)-x_{m}(k)\right)+v_{p}^{T}(k) R v_{p}(k)
$$

where $Q$ and $R$ are matrices to be chosen by the designer.

The performance index corresponding to the second structure includes terms weighting model-following error $\left(x_{p}-x_{m}\right)$, accumulated model-following error $x_{a}$ as defined in eq. 2.22, and the magnitude of the 
control $u_{p}$. This index, which therefore includes three weighting matrices $Q$, $S$, and $R$, is of the form:

$$
\begin{aligned}
\frac{1}{2} \sum_{k=0}^{\infty}\left(x_{p}(k)-x_{m}(k)\right)^{T} Q\left(x_{p}(k)-x_{m}(k)\right)+x_{a}^{T}(k) s \cdot x_{a}(k) \\
\quad+u_{p}^{T}(k) R u_{p}(k)
\end{aligned}
$$

Notice that if the matrix $S$ is all zeros, then no weight is placed on the accumulated plant-model error and this controller will no longer effectively have any feedback dynamics.

\subsubsection{Controller structure}

Infinite time optimal linear regulator theory was used to find the minimum of the performance indices constrained by the appropriate state equations. This results in a control formed by feeding back all states through constant gains. ${ }^{15}$ (Details are presented in the next section.) With this in mind, the specific controller structure implied by these performance indices may be described. Further, the performance indices and state equations for both cases may be put in terms of the infinite time optimal linear regulator problem:

Find $u(k)$ to minimize

$$
\frac{1}{2} \sum_{k=0}^{\infty} x^{T}(k) Q^{\prime} x(k)+u^{T}(k) R^{\prime} u(k)
$$

subject to

$$
x(k+1)=A x(k)+B u(k)
$$


For the first performance index which penalizes control rate rather than control magnitude (2.23), fig. 7 illustrates the final closed-ioop structure. Note that any control input (from the model state and control). or any plant state feedback signal must be "filtered" through the dynamic feedback controller before it affects the plant control. Reshaping the state equations into a form to which the optimal linear regulator results are directly applicable (2.25a and $2.25 b)$ gives:

$$
\begin{aligned}
& x(k)=\left[\begin{array}{c}
x_{p}(k) \\
u_{p}(k) \\
x_{m}(k) \\
u_{m}(k)
\end{array}\right] \quad u(k)=v_{p}(k) \quad A=\left[\begin{array}{cccc}
A_{p} & B_{p} & 0 & 0 \\
0 & I & 0 & 0 \\
0 & 0 & A_{m} & B_{m} \\
0 & 0 & 0 & I
\end{array}\right] \quad B=\left[\begin{array}{l}
0 \\
I \\
0 \\
0
\end{array}\right] \\
& Q^{\prime}=\left[\begin{array}{cccc}
Q & 0 & -Q & 0 \\
0 & 0 & 0 & 0 \\
-Q & 0 & Q & 0 \\
0 & 0 & 0 & 0
\end{array}\right] \quad R^{\prime}=R
\end{aligned}
$$

Note that the pilot's input (equal to the model control $u_{m}(k)$ ) is modeled as a constant - an assumption that does not overiy distort the reality of the situation and allows a complete analysis of the problem from a theoretical viewpoint.

The performance index that includes the accumalated model-following error (eq. 2.24) leads to a controller structure analogous. to the classical proportional-integral controller, as shown in fig. 8. Manipulating the state equations into a form to which the optimal linear regulator results are directly applicable, (2.25a and 2.25b) yields: 


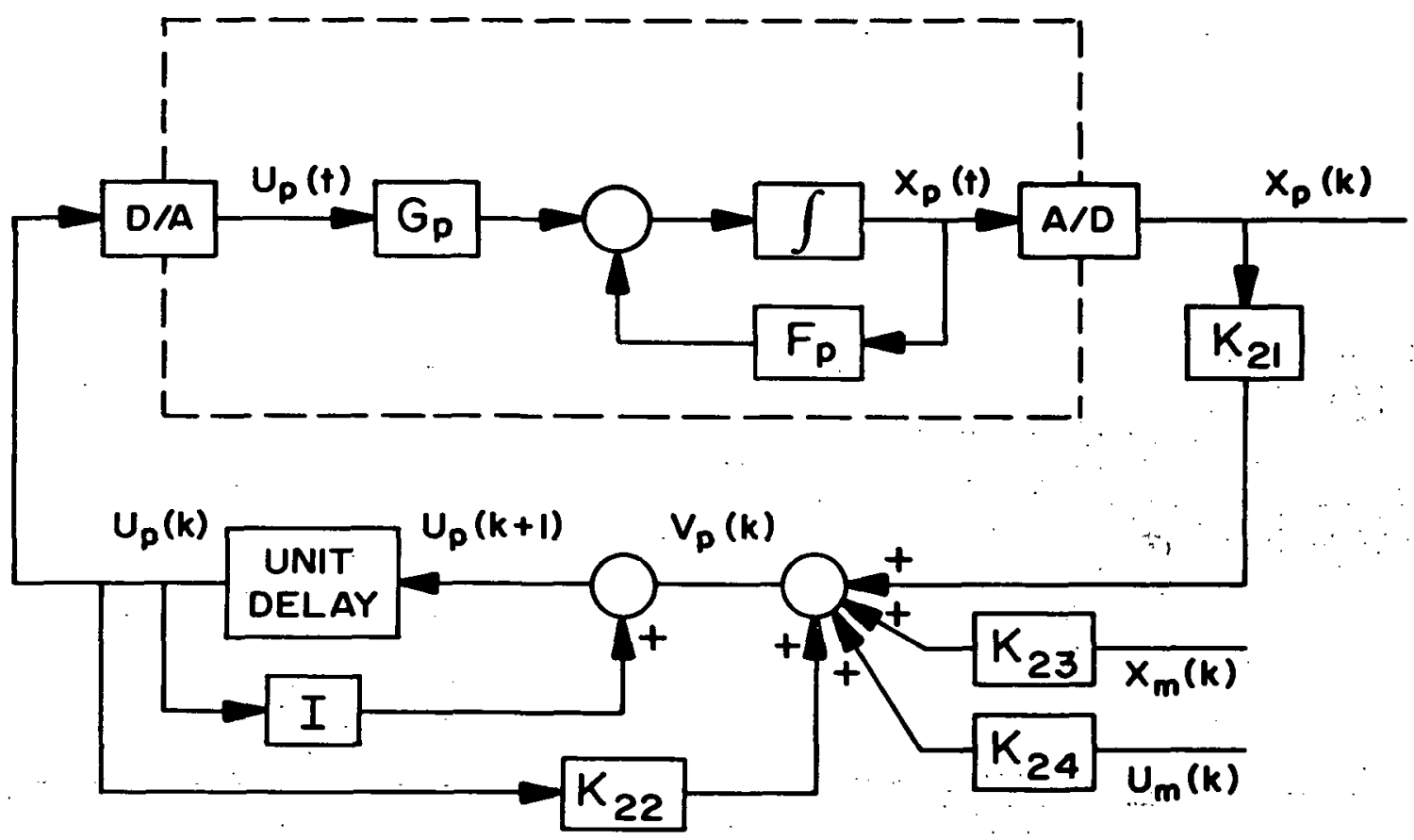

$$
\begin{aligned}
& x_{p}(t)=F_{p}(t) X_{p}(t)+G_{p}(t) U_{p}(t) \\
& U_{p}(k+1)=V_{p}(k)+U_{p}(k) \\
& V_{p}(k)=K_{21} x_{p}(k)+K_{22} U_{p}(k)+k_{23} x_{m}(k)+k_{24} U_{m}(k)
\end{aligned}
$$

FIG.7 Dynamic Feedback System Using "Control Rate" Feedback 


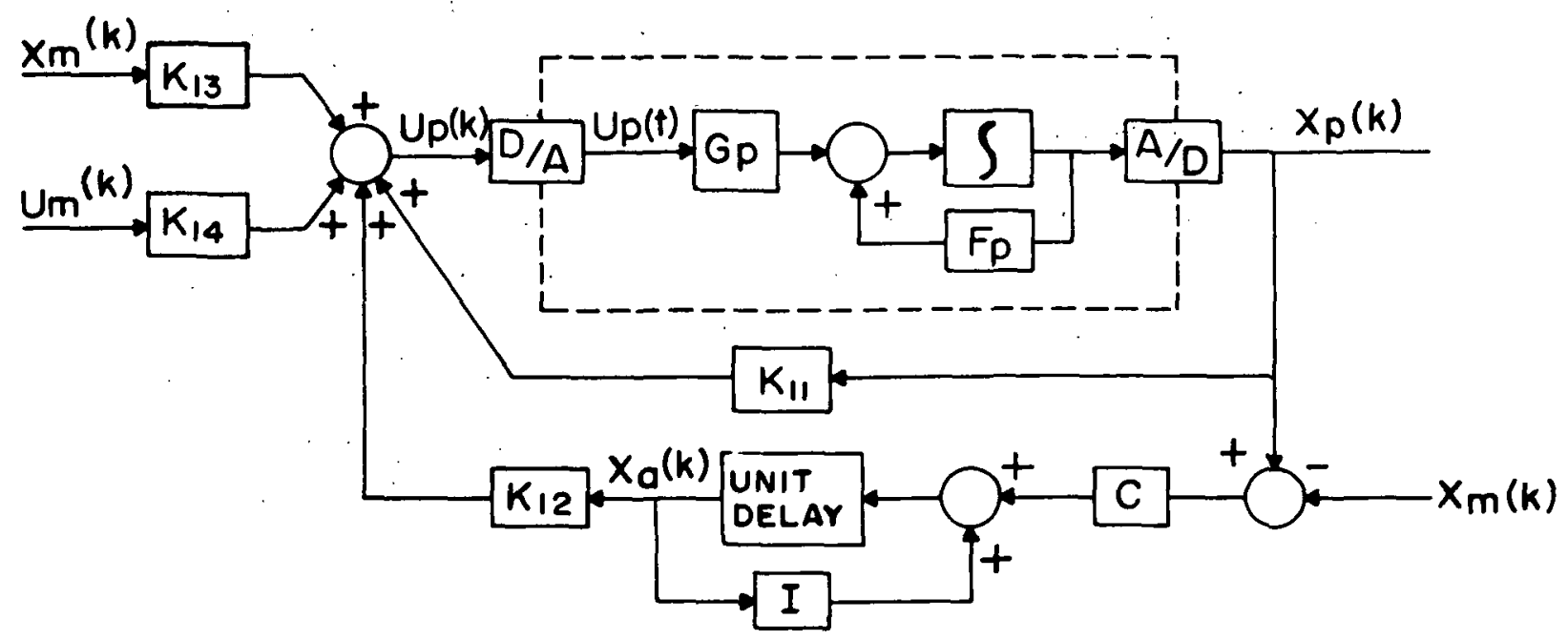

FIG. 8 DYNAMIC FEEDBACK SYSTEM USING "INTEGRAL ERROR"

\section{FEEDBACK}

$$
\begin{aligned}
& X_{p}(t)=F_{p}(t) X_{p}(t)+G p(t) \cup p(t) \\
& X_{a}(k+1)=X_{a}(k)+c\left(X_{p}(k)-X_{m}(k)\right) \\
& U p(k)=K_{11} X_{p}(k)+K_{12} X_{a}(k)+K_{13} X_{m}(k)+K_{14} U m(k)
\end{aligned}
$$


$x(k)=\left[\begin{array}{c}x_{p}(k) \\ x_{a}(k) \\ x_{m}(k) \\ u_{m}(k)\end{array}\right]$

$A=\left[\begin{array}{cccc}A_{p} & 0 & 0 & 0 \\ C & I & -C & 0 \\ 0 & 0 & A_{m} & B_{m} \\ 0 & 0 & 0 & I\end{array}\right] \quad B=\left[\begin{array}{c}B_{p} \\ 0 \\ 0 \\ 0\end{array}\right]$

$Q^{\prime}=\left[\begin{array}{cccc}Q & 0 & -Q & 0 \\ 0 & \therefore & 0 & 0 \\ -Q & 0 & Q & 0 \\ 0 & 0 & 0 & 0\end{array}\right] \quad R^{\prime}=R$

As noted above, if the weighting matrix $S$ were all zeros, then the feedback gain $\mathrm{K}_{12}$ in fig. 8 would be zero and the controller would be non-dynamic.

\subsubsection{Infinite Time Linear Optimal Regulator Solution The solution to the minimization of a quadratic.}

performance index:

$$
\frac{i}{2} \sum_{k=0}^{\infty} x^{T}(k) Q x(k)+u^{T}(k) R u(k)
$$

constrained by the state equation:

$$
x(k+1)=A x(k)+B u(k)
$$

is. well known and derived in Moore and Anderson ${ }^{15}$ among others. The control has the constant feedback structure:

$$
u(k)=-K x(k)
$$

where

$$
K=\left(B^{T} P B+R\right)^{-1} B^{T} P A
$$


and $P$ is specified by the steady state Riccati equation:

$$
P=Q+A^{T} P A-A^{T} P B\left(R+B^{T} P B\right)^{-1} B^{T} P A
$$

This nonlinear equation in $P$ may be solved in a number of ways. The simplest appears to be by iteration of the time varying Ricatti equation

$$
P(k+1)=Q+A^{T} P(k) A-A^{T} P(k) B\left(R+B^{T} P(k) B\right)^{-l} B^{T} P(k) A
$$

This equation converges to the solution for any positive definite initialization of $P(k)$ provided that the pair $[A, B]$ is controllable and the pair $[A, D]$ is observable (where $Q=D^{T} D$ ). At this point it should be noted that even though the model is not controllable, the solution will still exist because of the controllability of the plant. 16

The adaptation logic for this type controller is based upon an online iteration of eq. 2.31. Since the aircraft parameters vary continuously and relatively little within the anticipated gain update cycles, it can be expected that the exact solution to the corresponding steady state Riccati equation will not vary significantly between gain updates. Thus if at each gain update time, the Riccati equation is initialized with the most recent solution, it is hypothesized that it will be necessary to iterate only a few times to find the proper solution.

Finally as a practical matter, it should be noted that computation can be simplified if eq. 2.31 is partitioned taking into account the symetry of $P$ and the sparseness of the augmented system matrices $A$ and $B$ as defined by 2.26 and 2.27 . The resulting expressions can be found in eqs. $3.1,3.2$, and 3.3 . 


\subsection{Parameter Identification}

2.5.1 Introduction

Selection of a parameter identification algorithm for an

adaptive flight control system requires consideration of the following items:

- Required frequency of parameter updating

- Measurement noise characteristics

- The desirability of estimating true stability derivatives versus the elements appearing in the discrete transition matrix (These will be nonlinear functions of the stability derivatives.)

- Nonlinearities induced by simultaneous estimation of parameter and states

- Performance under closed loop control

If parameters vary relatively slowly, then a batch process such as quasilinearization (17) might be feasible. However, if updating must be done more frequently, then an online or recursive procedure with some sort of fading memory should be employed.

In designing parameter identification algorithms, attention must be given to the measurement noise characteristics. Whereas a relatively large variance necessitates the use of an identifier with a long memory to achieve smoothing, a small variance will enable the use of a short memory identifier that will be more-responsive to parameter variations. Further, it is conjectured that in a digital environment, greater success would be achieved if the unknown parameters of the discrete transition matrix itself. rather than the physical stability derivatives are identified. This follows because the discrete transition matrix is a highly nonlinear function of the stability derivatives making identification rather difficult. Furthermore computation of the discrete control law requires 
estimates for elements of the discrete transition matrix, and not of the stability derivatives. A precise description of the discrete parameter estimation problem is presented in Section 2.5.2.

In designing an identifier, consideration must be given to algorithms for estimating only parameters and algorithms for estimating both parameters and states simultaneously. Whereas the former set of algorithms utilizes the measurements to represent the true state, the latter set depends upon the augmentation of the unknown parameters to the system state vector. As shown in ref. (18), the combined state and parameter estimation problem is nonlinear because of the need to determine quantities that multiply each other (parameter times state). Consequently one must select a priming trajectory about which the system is to be linearized.

Typical algorithms for parameter estimation only include the weighted least squares approach and the stochastic gradient method. These are discussed in section 2.5.3. Section 2.5.4 discusses two approaches for estimating both parameters and states; namely, the extended Kalman filter and a decoupling process in which the linear state and parameter estimation problems are treated separately and alternately.

Finally, of importance is the performance of these identification procedures under closed-loop control. Because such control often results in transient behavior for only a very small amount of time and steady state behavior for a relatively large amount of time, there may not be sufficient excitation to allow accurate enough identification. Thus the need for an induced dither signal must be examined. Such dither could in fact be produced by feeding back for control computation the noisy state measurements themselves rather than filtered state estimates. In any event the performance 
of the identifier should ultimately be measured by the overall behavior of the adaptive control system rather than the individual estimates it produces for the various parameters. This follows from the fact that not all states are equally excited by any given pilot input command. Hence various motions will be completely decoupled or very insensitive to the specific values obtained for some of the parameters.

Thus while there may not be sufficient excitation present to accurately track a parameter, its value may not be very influential in determining the control signal needed for the maneuver being undertaken. Accurate tracking of all parameter continuously will only be possible if dither can be acceptably introduced into the motion of the aircraft.

Results of identifying under closed-loop control using the weighted least squares algorithm are presented in section 4.5 .

\subsubsection{Problem Statement}

The problem considered to date is that of determining the values of certain parameters appearing in the discretized aircraft equations of motions given exact measurements of the inputs and noisy measurements of the outputs. Noisy input measurements are to be considered in subsequent studies. Evaluation of the accuracy of this identification is to be based upon the resulting adaptive control loop performance.

As given by eq. 2.3, the lateral or longitudinal motion of the aircraft is represented by the vector difference equation

$$
\underline{x}_{p}(k+I)=A_{p}(q) \underline{x}_{p}(k)+B_{p}(\underline{q}) \underline{u}_{p}(k)
$$

where $\underline{q}$ is used to denote a vector whose elements are unknown parameters appearing in the plant matrices. For estimation purposes, it will be assumed 
that the system measurements can be described by

$$
\underline{y}_{p}(k)=H \cdot x_{-p}(k)+\eta(k)
$$

where: $\underline{\eta}(\mathrm{k})$ is a zero mean measurement noise sequence with covariance $\operatorname{matrix} \dot{R}$

$$
R(i, j)=\sigma_{i}^{2} \delta_{i j} \text {, where } \delta_{i j}=1 \text {, if } i=j \text {, and } \delta_{i j}=0 \text { otherwise }
$$

and: $\mathrm{H}$ is a selector matrix indicating just which states or combinations of states are measured. For the work considered to date, all states were assumed measurable, and hence $H=I$, the identity matrix.

Typical noise characteristics are included in Table I; these were provided by NASA - Langley Research Center. Although the specified noise is correlated, many of the experiments conducted to date have utilized uncorrelated (i.e., white) noise sequences, having the values cited in Table I, in order to simplify the analysis.

\subsubsection{Procedures for Parameter Estimation Only}

\subsubsection{Weighted Least Squares Parameter Identification}

The simple weighted least squares linear parameter

estimation scheme ${ }^{20}$ was chosen to avoid some of the problems of algorithms which estimate both parameters and states. Since noisy states are fed back through the control loop and used for parameter estimation, it is expected that some sort of state smoother will ultimately be necessary.

To apply this method to the systems defined by eqs. 2.32 and 2.33, it is necessary to differentiate between the set of parameters (in both $A_{p}$ and $B_{p}$ ) that are to be identified and the set of parameters that are not to be identified. 


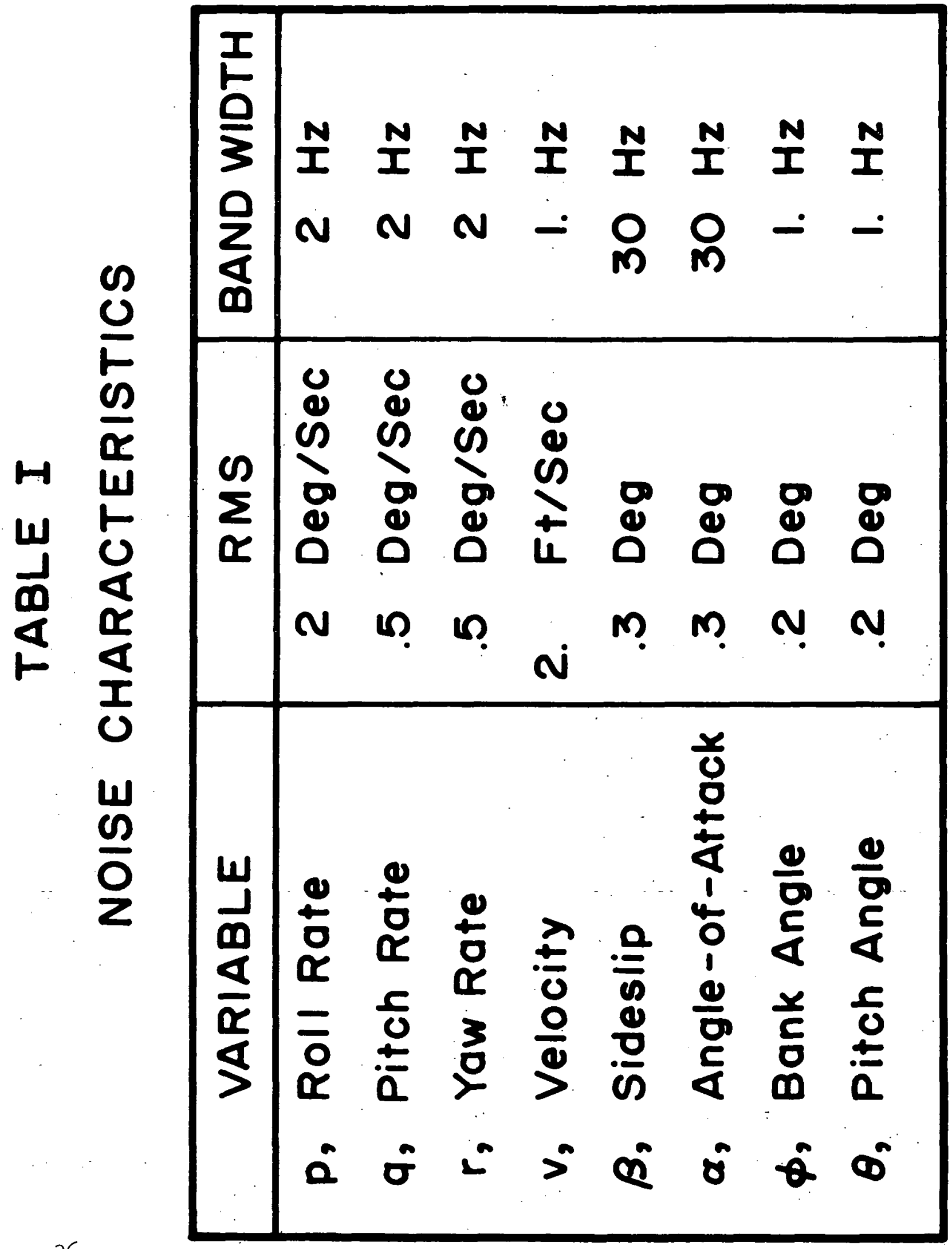


In particular a convenient representation for (2.32) results if all the parameters (both $a_{p}(i, j)$ and $b_{p}(i, j)$ ) which are to be identified are placed in a vector $\underline{q}$, and all those parameters which are not to: be identified are placed in a vector $\underline{s}$. With these definitions, the state equations can be rewritten as:

$$
\underline{x}(k)=C(k-I) \cdot \underline{q}+D(k-I) \underline{s}
$$

where $C_{i j}(k-1)$ contain the state or control measurement at the $(k-1)^{\text {st }}$-sample that couples $x_{i}(k)$ to $q_{j}$, and $D_{i j}(k-1)$. contains the state or control. measurement at the $(k-1)^{\text {st }}$ sample that couples $x_{i}(k) \cdots$ to $s_{j}$. A zero entry for a particular $C_{i j}$ (or $D_{i j}$ ) would indicate that no coupling exists between $x_{i}$ and $q_{j}$ (or between $x_{i}$ and $s_{j}$ ).

Defining the estimate formed on the basis of $k$

samples for the parameter vector as $q(k)$, the weighted least squares algorithm as developed by Young ${ }^{20}$ and Mendel $^{21}$ minimizes the performance index:

$$
J=\sum_{i=0}^{k}(y(i)-\hat{x}(i))^{T} R^{-l}(\underline{y}(i)-\hat{x}(i))
$$

where $\underline{y}(i)$ is the actual measurement made of $\underline{x}(i)$

and $\hat{\hat{x}}(i)=C(i-I) \hat{q}(k)+D(i-1) \underline{s}$

i.e., $\hat{x}(i)$ is the "estimate" of $\underline{x}(i)$ formed by substituting $\hat{q}(k)$ into the state equation (2.35).

The weighting matrix $R^{-1}$ is usually selected to be a diagonal matrix whose elements $r_{i i}^{-1}$ reflect either the confidence and/or the relative magnitudes of each of the measurements $y_{i}$. Furthermore a fading factor of the form $\left.\alpha^{2 i}(\alpha) I\right)$ can be included in $R^{-1}$ in order to give major 
emphasis to the most recent measurements. 25,26

$$
\text { Minimization of (2.36) yields }{ }^{20} \text { : }
$$

$$
\hat{\mathrm{q}}(\mathrm{k})=\mathrm{P}(\mathrm{k}) \mathrm{B}(\mathrm{k})
$$

where $\left.P(k)=\left[\sum_{\ell=1}^{k} c^{T}(\ell-I)\right) R^{-1} c(\ell-I)\right]^{-I}$

and $\quad B(k)=\sum_{\ell=1}^{k} C^{T}(\ell-I) R^{-I}[y(l)-D(\ell-I) \underline{s}]$

Note that computation of the estimate $\hat{q}(k)$ from these formula requires at each new sample a matrix inversion of order equal to the number of parameters being estimated. This solution however can be rewritten in a recursive manner that takes into account the effects of an additional measurement in a manner so as to reduce the matrix inversion requirements. In particular, the estimate $\hat{q}(k)$, based upon $\mathrm{k}$ samples, can be written in terms of $\hat{q}(k-l)$, the estimate based upon $k-1$ samples using the equations: 20

$$
\begin{aligned}
& \hat{q}(k)=\hat{q}(k-1)-K(k)(C(k-1) \hat{q}(k-1)-z(k)) \\
& z(k)=y(k)-D(k-1) s \\
& K(k)=P(k-1) C^{T}(k-1)\left(R+C(k-1) P(k-1) C^{T}(k-1)\right)^{-1} \\
& P(k)=P(k-1)-K(k) C(k-1) P(k-1)
\end{aligned}
$$

These equations are solved recursively given an initial value for the $P$ matrix, $P(0)$, and an initial value for the estimates, $\hat{q}(0)$. Mendel ${ }^{21}$ showed that if the expected value of the measurement noise is zero (i.e., $E(\eta(k))=0$ ) and if and only if $c(k)$ is free of measurement errors, then the parameter estimates will be unbiased in 
the sense that

$$
E(\hat{q}(k))=q
$$

The latter condition is clearly not applicable in the aircraft identification problem since $\mathrm{C}(\mathrm{k})$ is indeed constructed from noisy samples of the states. Then a biased estimate for $\mathrm{q}$ is anticipated.

Furthermore if the above condition did hold and if $R$, the weighting matrix in 2.36 were selected to be the covariance of the actual measured value of

$$
x(k)-D(k-I) \underline{s}
$$

then $q(k)$ as computed from 2.37 would be the Iinear minimum variance estimate for $q$, and $P(k)$ would be the covariance for this estimate.

$$
\text { Despite the inability to obtain a minimum variance }
$$

using eq. 2.37, the diagonal elements of $R, r_{i i}$, were in practice set equal to the variance in

$$
y_{i}(k)-\sum_{j} D_{i j}(k-1) s_{j}
$$

i.e.

$$
r_{i i}=\sigma_{i}^{2}+\sum_{j} \sigma_{j}^{2} s_{j}^{2}
$$

where $j$ ranges over those indices for which $D_{i j j} \neq 0$, and as in eq. 2.33 $\sigma_{i}^{2}$ is the variance of the disturbance inherent in $y_{i}$, the measurement of $\mathbf{x}_{\mathbf{i}}$

To take into account the time variations in the parameters due to the aircraft maneuvers, either the fading factor $\alpha^{2 k}$ can be incorporated or the equations can be rederived with the parameter variations 
modeled as fictitious noise sequences, $\sum_{j}^{\leqslant}(k) \cdot{ }^{20}$ Such a representation is:

$$
\underline{q}(k+1)=\underline{q}(k)+\xi(k), \operatorname{cov}(\xi)=Q
$$

Practically speaking, this artifice causes the filter to continually track the parameters. The corresponding recursive scheme is sjmilar to that of 2.37 , but now includes an update of the matrix. $P(k)$ each sample as given in:

$$
\begin{aligned}
& \hat{q}(k)=\hat{q}(k-1)-K(k)(C(k-1) \hat{q}(k-1)-z(k)) \\
& z(k)=y(k)-D(k-1) s \\
& P(k / k-1)=P(k-1)+Q \\
& K(k)=P(k / k-1) C^{T}(k-1)\left(R+C(k-1) P(k / k-1) C^{T}(k-1)\right)^{-1} \\
& P(k)=P(k / k-1)-K(k) C(k-1) P(k / k-1)
\end{aligned}
$$

The matrix $Q$ has the effect of keeping $P(k)$ from getting so small that parameter updating becomes insignificant due to a small gain matrix $K(k)$. Note that at steady state $P(k)$ will go to a fixed value determined by $Q$. Selection of the elements of $Q$ requires a trade-off between a filter that gives erroneous parameter estimates due to tracking of the measurement noise (if the elements of $Q$ are too large) and a filter that just smooths out the parameter variations (if the elements of $Q$ are too small). Computationally, the matrix inversion can be expected to cause trouble. However, if $R$ and $Q$ are chosen to be diagonal and if $P(k)$ is initialized as a diagonal matrix, the term $C(k-1) P(k / k-1) C^{T}(k-1)$ will be diagonal since $c(k-1)$ contains only one nonzero. entry' in each column. The corresponding matrix inversion is thus reduced to scalar divisions. 
Details pertaining to the tuning of $Q$ and $P(O)$

are given in section 4.2.1.

\subsubsection{Stochastic Gradient Estimation}

In view of the fact that the weighted least squares algorithm ignores the noise present in the matrix $c(k-1)$ in eq. (2.35) and consequently produces a biased estimate, a stochastic gradient procedure is being considered in anticipation of its capabilities for producing unbiased estimates. $^{21}$ To illustrate its application, consider the $i^{\text {th }}$ row of eq. 2.35, i.e.,

$$
x_{i}(k)=\sum_{j} c_{i j}(k-1) q_{j}+\sum_{j} D_{i j}(k-I) s_{j}
$$

To simplify the details it will be assumed that no term in the $i^{\text {th }}$ row of $\mathrm{C}(\mathrm{k})$ is zero. Trivial modifications can be incorporated if some of these terms are in fact zero:

Eq. 2.42 is now rewritten as:

$$
x_{i}(k)-\sum_{j} D_{i j}(k-1) s_{j}=\sum_{j} c_{i j}(k-1) q_{j}
$$

At this point it should be noted that since $D_{i j}(k-1)$ is either null, or is the state or control that couples $x_{i}$ to $s_{j}$, a parameter not requiring identification, the left hand side of (2.43) is a linear combination of measurable quantities. Consequently, a measurement $z(k)$ for the left hand side of (2.43) can be constructed by replacing $x_{i}(k)$ and $x_{i}(k-1)$ by the respective corresponding noisy measurements $y_{i}(k)$ and $\mathrm{y}_{i}(\mathrm{k}-1)$. Thus

$$
z_{i}(k)=x_{i}(k)-\sum_{j} D_{i j}(k-1) s_{j}+Y(k)
$$


where $\gamma(k)$ contains the noise inherent in the measurement of $x_{i}(k)$ and in the measurement of those states necessary for forming $\sum_{j} D_{i j}(k-1) s_{j}$.

$$
\text { If } C_{i j}(k-1) \text { in (2.43) is now replaced by its }
$$

corresponding measurement, then the following approximation model results.

$$
\hat{z}_{i}(k) \approx \sum_{j} r_{i j}(k-1) q_{j}
$$

where $r_{i j}(k-1)$ is the actual measured value for $c_{i j}(k-1)$.

Given this approximation model and assuming

uncorrelated noise sequences, the unbiased stochastic gradient algorithm for estimating $q_{i}$ is: 21

$$
\hat{q}_{i}(k+I)=\left[I+W(k) \sum\right] \hat{q}_{i}(k)+W(k) r_{i}(k) \tilde{z}_{i}(k)
$$

where:

$\hat{q}_{i}(k)$ is the estimate for $q_{i}$.

$$
\tilde{z}_{i}(k)=z_{i}(k)-\sum_{j} r_{i j}(k-1) \hat{q}_{j s}
$$

$r_{i}(k)$ is the vector with components $r_{i j}(k)$

$$
\sum \text { is the covariance of th }
$$

and $\mathrm{W}(\mathrm{k})$ is a weighting matrix.

To insure convergence $W(k)$ must be of the form:. 21

$$
w(k)=P(k) \text { diag }\left[h_{1}(k), \ldots, h_{l_{i}}(k)\right]
$$


where:

$$
\begin{aligned}
& 0 \leq h_{L} \leq h_{i} \leq h_{2}<\infty \\
& \rho(k)>0 \\
& \sum_{k=0}^{\infty} \rho_{(k)} \rightarrow \infty
\end{aligned}
$$

and

$$
\sum_{k=0}^{\infty} \rho_{(k)}^{2}<\infty
$$

These conditions essentially enable the procedure to be able to correct the parameter estimates as much as needed even for large values of $k$. Again because no simultaneous estimation of states and parameters is involved the algorithm is strictly linear.

2.5.4 Procedure for Simultaneous State and Parameter Estimation Algorithms are now presented that perform both state and parameter estimation. Those considered are the Extended Kalman filter and a decoupling process in which linear state estimation and linear parameter identification are performed separately and alternately.

\subsubsection{Extended KaIman Filter}

This section presents the general formula used in Kalman filter estimation without any attempt for verification. Detailed derivations may be found in ref. (23) or any complete text dealing with filtering and estimation theory.

The system defined by eqs. 2.32 and 2.33 will be used for the presentation of the identification algorithm. If only state 
estimation were to be performed, then the Kalman estimate $x_{p}(k)$ for the state would be both a minimum variance estimate and a least squares estimate in the sense of minimizing the performance function (18)

$$
J_{n}=\sum_{k=0}^{n}\left[\underline{y}_{p}(k)-H \hat{x}_{p}(k)\right]^{T} R^{-1}\left[\underline{y}_{p}(k)-H \stackrel{\hat{x}}{p}_{p}(k)\right]
$$

where $R$ is the covariance of the measurement noise $n$. However if the measurement noise covariance matrix $R$ is not known explicitly and/or the noise is not Gaussian, then the estimate is optimal in the sense of minimizing

$$
J_{n}=\sum_{k=0}^{n}\left[\underline{y}_{p}(k)-H \hat{x}_{p}(k)\right]^{T} R_{e}^{-1}\left[\underline{y}_{p}(k)-H \hat{x}_{p}(k)\right]
$$

when $R_{e}$ is the estimated value of $R$. This estimate will not however be optimal in the sense of minimizing the variance of the error.

$$
\begin{aligned}
\text { The estimated state } \hat{x}_{p}(k) \text { is given by: } \\
\hat{\underline{x}}_{p}(k)=A_{p} \hat{\underline{x}}_{p}(k-I)+B_{p} \underline{u}_{p}(k-I)+ \\
K(k)\left[\underline{y}_{p}(k)-H\left[A_{p} \hat{x}_{p}(k-I)+B_{p} \underline{u}_{p}(k-I)\right]\right]
\end{aligned}
$$

where:

$$
\begin{aligned}
& A_{p} \hat{x}_{p}(k-I)+B_{p} \underline{u}_{p}(k-1) \text { is the "extrapolated" state. } \\
& H\left[A_{p} \hat{x}_{p}(k-I)+B_{p} \underline{u}_{p}(k-I)\right] \text { is the "extrapolated" observation, }
\end{aligned}
$$

and

$\mathrm{K}(\mathrm{k})$ is the gain matrix defined as:

$$
\begin{aligned}
& K(k)=\left[M(k) H^{T}\right]\left[\begin{array}{ll}
H M(k) & H^{T}+R
\end{array}\right]^{-1} \\
& M(k) \text { is the "extrapolated covariance matrix of }
\end{aligned}
$$

$\hat{x}_{p}(k)$ given the observations $y_{p}(k-1), y_{p}(k-2), \ldots y_{p}(0)$ and is computed 
recursively as:

$$
M(k+1)=A P(k) A^{T}
$$

$P(k)$ is the covariance of the estimate $\hat{x}_{p}(k)$ given $y_{p}(k), y_{p}(k-1), \ldots$ $\mathrm{y}_{\mathrm{p}}(0)$; that is

$$
P(k)=E\left\{\left[\underline{\underline{x}}(k)-\underline{x}_{p}(k)\right]\left[\hat{x}_{p}(k)-\underline{x}_{p}(k)\right]^{T} \mid \underline{y}_{p}(k), \ldots \underline{y}_{p}(0)\right\}
$$

and can be computed from $\mathrm{M}(\mathrm{k})$ and $\mathrm{K}(\mathrm{k})$ as :

$$
P(k)=M(k)-K(k) H M(k)
$$

Up to this point, it has been demonstrated how Kalman filtering can be used to estimate the plant state vector $\hat{\mathrm{x}}_{\mathrm{p}}$.

To estimate any unknown parameters $q_{i}$ appearing in the state transition matrix $A_{p}$ and the gain matrix $B_{p}$ it is necessary to form an augmented state vector. This is done by appending to the state equations the set of difference equations

$$
q_{i}(k+1)=q_{i}(k) \quad i=1, \ldots, p
$$

or in vector form

$$
\underline{q}(k+1)=\underline{q}(k)
$$

Thus the augmented system becomes:

$$
\begin{aligned}
\underline{x}^{a}(k+l) & =A^{a}\left(\underline{x}^{a}(k), k\right)+B\left(\underline{x}^{a}(k), k\right) \underline{u}(k) \\
\underline{y}(k) & =H^{a}(k) \underline{x}^{a}(k)+h(k)
\end{aligned}
$$

where

$$
\begin{aligned}
& \underline{\mathrm{x}}^{\mathrm{a}}=\text { augmented state vector, given by } \\
& \quad \underline{\mathrm{x}}^{\mathrm{a}} \cdot=\left[\begin{array}{c}
\mathrm{x}_{\mathrm{p}} \\
\mathrm{p} \\
\mathrm{q}
\end{array}\right]
\end{aligned}
$$


$A^{a}=$ matrix of the augmented system given as

$$
A^{a}=\left[\begin{array}{c:c}
A_{p}(q) & N_{1} \\
\hdashline N_{2} & I_{p}
\end{array}\right] .
$$

$\mathrm{H}^{\mathrm{a}}=$ augmented system output matrix given by

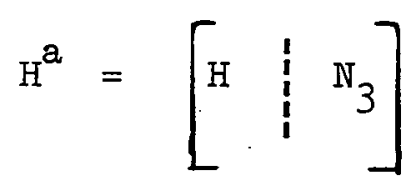

$I_{p}$ is an identity matrix of dimension $(p \times p)$, and $N_{1}, N_{2}$ and $N_{3}$ are null matrices with dimensions of $(n \times p),(p \times n)$ and $(n \times p)$ respectively.

Time variations in the parameters can be accounted

for by including a fictitious noise component $\xi_{i}(k)$ in eq. 2.53 such that: 20

$$
q_{i}(k+1)=q_{i}(k)+\xi_{i}(k)
$$

where the variance and degree of correlation in $\xi_{i}(k)$ are to be indicative of the anticipated fluctuation in q. A particularly useful assumption is to define $\xi(k)$ as an uncorrelated vector sequence with zero mean and covariance $Q$. This term would thin be added to the predicted covariance defined in eq. 2.51 giving:

$$
M(k+1)=A \quad P(k) \quad A^{T}+Q
$$

An alternate method for tracking time varying parameters is to introduce a fading on the filter's memory by replacing the weighting matrix $R_{e}$ appearing in eq. 2.48 by: 25,26

$$
\mathrm{R}(\mathrm{k})=\alpha^{-2 \mathrm{k}} \mathrm{R}_{\mathrm{e}} \quad \alpha>\mathrm{I}
$$


where $\alpha$ the fading factor is typically between 1.0 and 1.1 .

Note that the augmented system, eq. 2.54 is nonlinear and, therefore, incompatible. with the previous formula. This nonlinear equation must therefore be linearized about a nominal or model trajectory $x_{n}$. State vector and parameter estimates will then be given in terms of the incremental variations about this nominal trajectory or:

$$
\hat{\underline{x}}_{\underline{p}}(k)=\underline{x}_{N}(k)+\Delta \hat{\underline{x}}(k)
$$

and

$$
\hat{\hat{q}}(k)=q_{N}(k)+\Delta \underline{\hat{q}}(k)
$$

where $\underline{x}_{\mathrm{N}}$ and $\underline{q}_{N}$. are the state and parameters corresponding to the nominal or model system, and $\Delta \hat{\underline{x}}_{p}(k)$ and $\Delta \hat{A}_{\underline{q}}(k)$ are the corresponding incremental quantities. Iinearizing (2.54) about the nominal augmented state vector $\left(x_{N}, q_{N}\right)^{T}$ yields :

$$
\left[\begin{array}{ll}
\Delta \underline{x}_{p}(k+1) \\
\Delta \underline{q}(k+1)
\end{array}\right]=J(k)\left[\begin{array}{cc}
\Delta \underline{x}_{p}(k) \\
\Delta \underline{q}(k)
\end{array}\right]
$$

where $J(k)$ is the Jacobian matrix:

$$
J(k)=\left[\begin{array}{c:cc}
A\left(\underline{q}_{N}(k)\right) & \frac{\partial}{\partial q}\left(\underline{A x}+\underline{B u}_{-}\right. & \\
& \underline{x}_{N}(k) & \underline{q}_{N}(k) \\
\hdashline & I_{p}
\end{array}\right]
$$

The extended Kalman filter equation applied to the system defined by $(2.54)$ is thus given by ${ }^{(23)}$ : 


$$
\left[\begin{array}{c}
\Delta \hat{\underline{x}}_{p}(k+1 / k+1) \\
\Delta \hat{\underline{q}}_{p}(k+1 / k+1)
\end{array}\right]=\left[\begin{array}{l}
\Delta \hat{\underline{x}}_{p}^{(k+l / k)} \\
\Delta \hat{\hat{q}}(k+1 / k)
\end{array}\right]+K(k)\left[\Delta \underline{y}_{p}(k)-H \Delta \underline{\underline{x}}_{p}(k+1 / k)\right]
$$

where:

$$
\begin{gathered}
\Delta \underline{\hat{x}}, \Delta \underline{\hat{q}} \text { are the estimated incremental variations in the state } \\
\text { vector and parameters. }
\end{gathered}
$$

$$
\Delta y_{p}(k)=\underline{y}_{p}(k)-\underline{y}_{\underline{N}}(k)
$$

and

$$
\left[\begin{array}{ll}
\Delta \underline{\hat{x}}_{p}(k+1) / k \\
\Delta \underline{\hat{q}}(k+1) / k
\end{array}\right]=J(k)\left[\begin{array}{l}
\Delta \underline{\hat{x}}_{\underline{p}}(k / k) \\
\Delta \underline{\hat{q}}(k / k)
\end{array}\right]
$$

Estimates for states and parameters at time $k$ would thus be given by:

$$
\begin{aligned}
& \hat{\underline{x}}_{\mathrm{p}}(k+I / k)=\underline{x}_{N}(k+1)+\Delta \hat{\underline{x}}_{p}(k+I / k) \\
& \hat{\underline{q}}(k+I / k)=\underline{q}_{N}+\Delta \underline{\hat{q}}(k+I / k)
\end{aligned}
$$

$$
\text { With regard to defining the model trajectory, the }
$$

following possibilities should be considered:

\section{Procedure 1:}

The model or nominal trajectory can be defined by specifying the initial parameter vector $q_{N}(0)$, and the initial state vector $x_{N N}(0)$. These in turn define the solution to:

$$
\begin{aligned}
& \underline{x}_{-N}(k+1)=A_{N N} \underline{x}_{-N}(k)+B_{N} \quad \underline{u}_{p}(k) \\
& \underline{y}_{N N}(k)=H \quad \underline{x}_{N}(k)
\end{aligned}
$$


where $\quad \underline{x}_{N}=$ state vector of the nominal trajectory

$A_{\mathrm{N}}=$ system matrix defined by the initial value of the parameters, $\hat{\mathrm{q}}_{\mathrm{N}}(0)$.

$B_{N}=$ gain vector defined by the initial value of the parameters, $\hat{\mathrm{q}}_{\mathrm{N}}(0)$.

- The matrix $: J(k)$ is then updated as follows using this nominal trajectory $x_{N}$ and the initial value of the parameters:

$$
J(k)=\left[\begin{array}{c:c}
A_{N}\left(q_{N}\right) & \left.\frac{\partial}{\partial q}\left(\dot{A ~ X}_{N}+B u_{p}\right)\right|_{q_{N}} \\
\hdashline 0 & I_{P}
\end{array}\right]
$$

\section{Procedure 2:}

The nominal trajectory is defined using the previous estimate of the state to initialize the model equations at each step, i.e.,

$$
\underline{x}_{N}(k+1)=A_{N} \underline{\hat{x}}_{p}(k / k)+B_{N} \quad \underline{u}_{p}(k)
$$

As in the previous case $x_{N}(k+I)$ is used to compute $J(k)$ from eq. 2.58.

\section{Procedure 3:}

The estimate $\hat{\mathrm{q}}(\mathrm{k} / \mathrm{k})$ is used to replace the model parameter vector ${ }^{\mathrm{q}} \mathrm{N}$, such that:

$$
\underline{x}_{-N}(k+1)=A_{p}(\underline{\hat{q}}(k)) \underline{x}_{\underline{N}}(k)+B_{p}(\underline{\hat{q}}(k)){\underset{u}{p}}_{p}(k),
$$

and $J(k)$ is subsequently computed using $x_{N}(k)$ and $\hat{q}(k)$ as: 


$$
J(k)=\left[\begin{array}{c:c}
A_{N}(\hat{q}(k)) & \frac{\partial}{\partial q}\left(A_{N}+B u_{p k}\right)_{\hat{q}} \\
\hdashline 0 & I
\end{array}\right]
$$

Procedure 4:

The equations are linearized about the most recent estimates for both. $q_{k}$ and $\underline{x}_{\mathrm{K}} \cdot$ Thus

$$
\begin{aligned}
& \underline{x}_{-N}(k+I)=A_{p}(\underline{\hat{q}}(k)) \underline{\hat{x}}_{p}(k / k)+B_{p}(\underline{\hat{q}}(k)) \underline{u}_{p}(k) \\
& q_{\mathrm{N}}(k+I)=\hat{q}(k+1 / k+I)
\end{aligned}
$$

This summarizes the necessary equations for the extended Kalman filter for state estimation and/or parameter identification. Details of the numerical experiments. condacted are presented in section 4.2 .

\subsubsection{Separate Linear State and Parameter Estimation} (SLSPE)

The separate linear state and parameter estimator (SLSPE) as suggested by Graupe ${ }^{24}$ is a suboptimal decoupling process in which linear state estimation and linear parameter identification are performed separately and alternately. Because each estimation process is basically a recursive weighted least squares algorithm, the procedures are basically those detailed in section 2.5.3.1.

To define the procedure consider again the linear system:

$$
\begin{gathered}
\underline{x}_{p}(k+1)=A_{p} \underline{x}_{p}(k+1)+B_{p} \underline{u}_{p}(k+1) \\
\underline{y}_{p}(k)=\underline{x}_{p}+\underline{n}
\end{gathered}
$$


when $A_{p}$ and $B_{p}$ contain unknown parameters.

Define two linear systems, the state system defined by eq. (2.66a) and measured by eq. 2.66b, and the parameter system:

$$
\underline{q}(k+1)=\underline{q}(k)
$$

measured by eq. 2.35. Clearly these two systems are coupled through the system state $\underline{x}_{p}$ and the parameter vector $\underline{q}$.

In order to decouple these systems, states are to be estimated by assigning some value to the parameter vector $\hat{q}$ and minimizing:

$$
J_{s}=\therefore \sum_{k=0}^{N}\left(y_{p}(k)-\hat{x}_{p}(k)\right)^{T} R_{s}^{-1}\left(y_{p}(k)-\hat{x}_{p}(k)\right)
$$

subject to the satisfaction of eq. 2.66 .

Parameters are in turn to be estimated by assigning a value to the state vector $x_{p}$ and minimizing:

$$
J_{p}=\sum_{k=0}^{N}\left(y(k)-\hat{x}_{p}(k)\right) R_{p}^{-1}\left(y(k)-\hat{x}_{p}(k)\right)
$$

where:

$$
\hat{x}_{p}(k+I)=A_{p}(\hat{q}) \hat{x}_{p}(k)+B_{p}(\hat{q}) u_{p}(k)
$$

It is thus seen that the estimators for the states and the parameters are the algorithms defined respectively by eq. 2.49 and eq. 2.37. In summary then, the SLSPE procedure is separable into the following two distinct problems: 
A. At time $k$ estimate $\hat{x}_{p}(k / k)$, using for $q$
its most recent estimate $\hat{q}_{(k-1 / k-1)}$
A. At time $k$ estimate $\hat{q}(k / k)$, using for $x_{p}$
its most recent estimate, i.e., $\hat{x}_{p}(k / k)$

It is seen that the proposed procedure is suboptimal in the sense that the two objective functions are minimized separately and not simultaneously, resulting in the need to alternate between a linear state estimation problem and a linear parameter estimation problem. 


\subsection{Considerations Conceming the Adaptive Algorithms}

When designing an algorithm to be implemented, practical considera-

tions must be very influential on the trade-off between accuracy and simplicity. In a digital adaptive flight controller, one of the prime practical restrictions is the size and speed of the on-line digital computer. This will affect both the timing and storage requirements of the adaptive algorithm. Figure 9 depicts the block diagram for an overall adaptive control system, and fig. 10 illustrates the various timing intervals involved in the realization.

The smallest timing interval is the control computation interval $\left(T_{c}\right)$ and typically is between 0.03 and 0.20 seconds.. State estimation, if used, must be as fast, since the state is used by the feedback controller in calculating the new control. The two larger intervals are required for gain adaptation. The parameter identification interval $\left(T_{i}\right)$ must not be longer than the gain update interval $\left(\mathrm{T}_{\mathrm{g}}\right)$, since the gain update algorithm requires the new parameter estimates. If parameter estimation alone is performed, then it is possible to have $T_{i}>T_{c}$; however, if states and parameters are to be estimated simultaneously, then it is necessary that $T_{i}=T_{c} \cdot$ Gain update intervals $\left(T_{g}\right)$ being considered range from 1.0 to 10.0 seconds. Since storage requirements vary from algorithm to algorithm, they must be taken into account. Recursive gain update, parameter identification and'state estimation algorithms will require storage of the various matrices used in their formulation.

Attention must be given to methods for reducing the complication of these algorithms. Prime among these is the identification of only the most sensitive parameters of the aircraft, the others being set at some representa- 


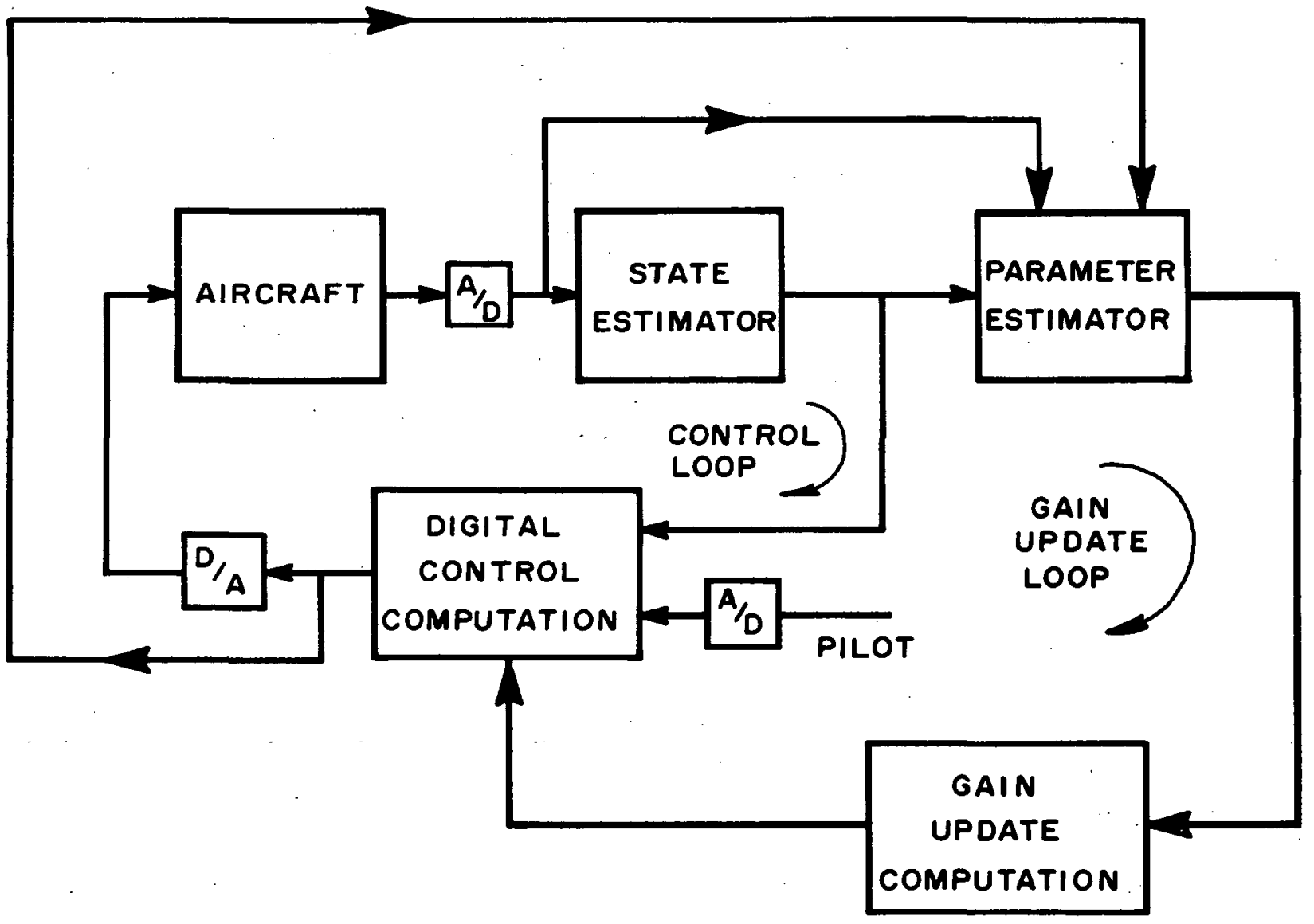

FIG.9 ADAPTIVE CONTROLLER STRUCTURE 

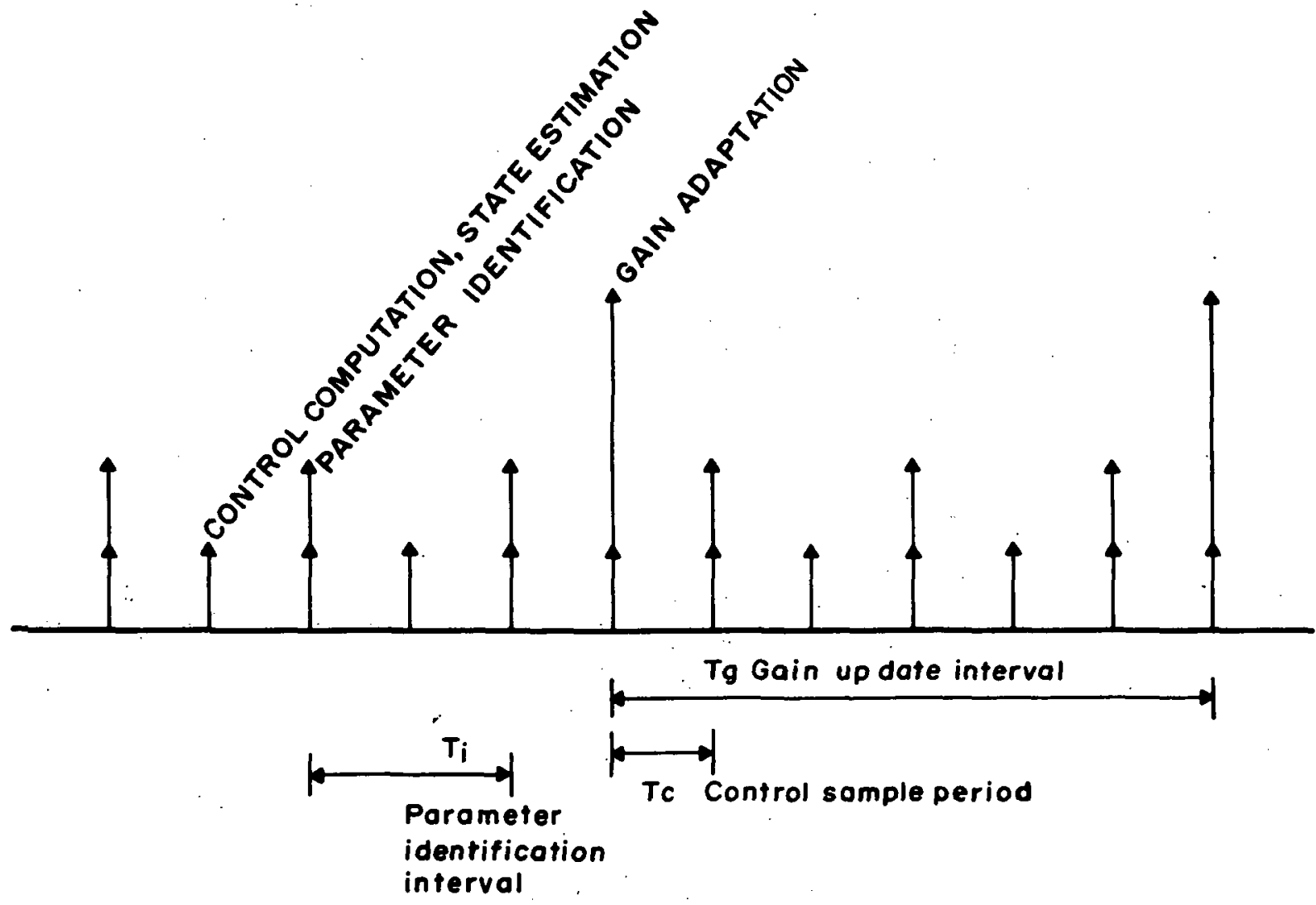

FIG. 10 TIMING CHART FOR ADAPTIVE CONTROL 
tive nominal value. The specific parameters to be identified are found according to the influence errors in identification have on the state. The sensitivity study performed for this purpose is described in section 4.2 . Furthermore, algorithms can be simplified by taking advantage of the sparseness of many of the matrices involved. This leads to a natural partitioning of the problem into a group or series of simpler problems. A further simplification may be made for calculations involving symetric matrices, although the extra information could be used to achieve more accurate calculations.

Structural considerations for implementing digital adaptive control systems based upon the single stage algorithm and linear quadratic regulator Iogic are next presented in Sections 3.2 and 3.3 respectively.

\subsection{Adaptive Logic Based Upon Single Stage Optimization Procedures} Because of the characteristics outlined in Section 2.4.2, the controller based upon the single stage index which penalizes the next stage error between plant and model was chosen for implementation in an adaptive control Ioop.

The control law is derived based upon the minimization of a weighted combination of the instantaneous squared error between the states of a linear plant and model at the next stage and the present control energy. The result is a control signal that is a linear combination of plant and model states and model inputs as given in 2.4.2. The explicit formula for computing the control gains are show at the bottom of fig. 11. In order to satisfy the identification requirements, an on-line weighted least squares identifier, described by eq. 2.41 has been interfaced with this controller. The results of the 


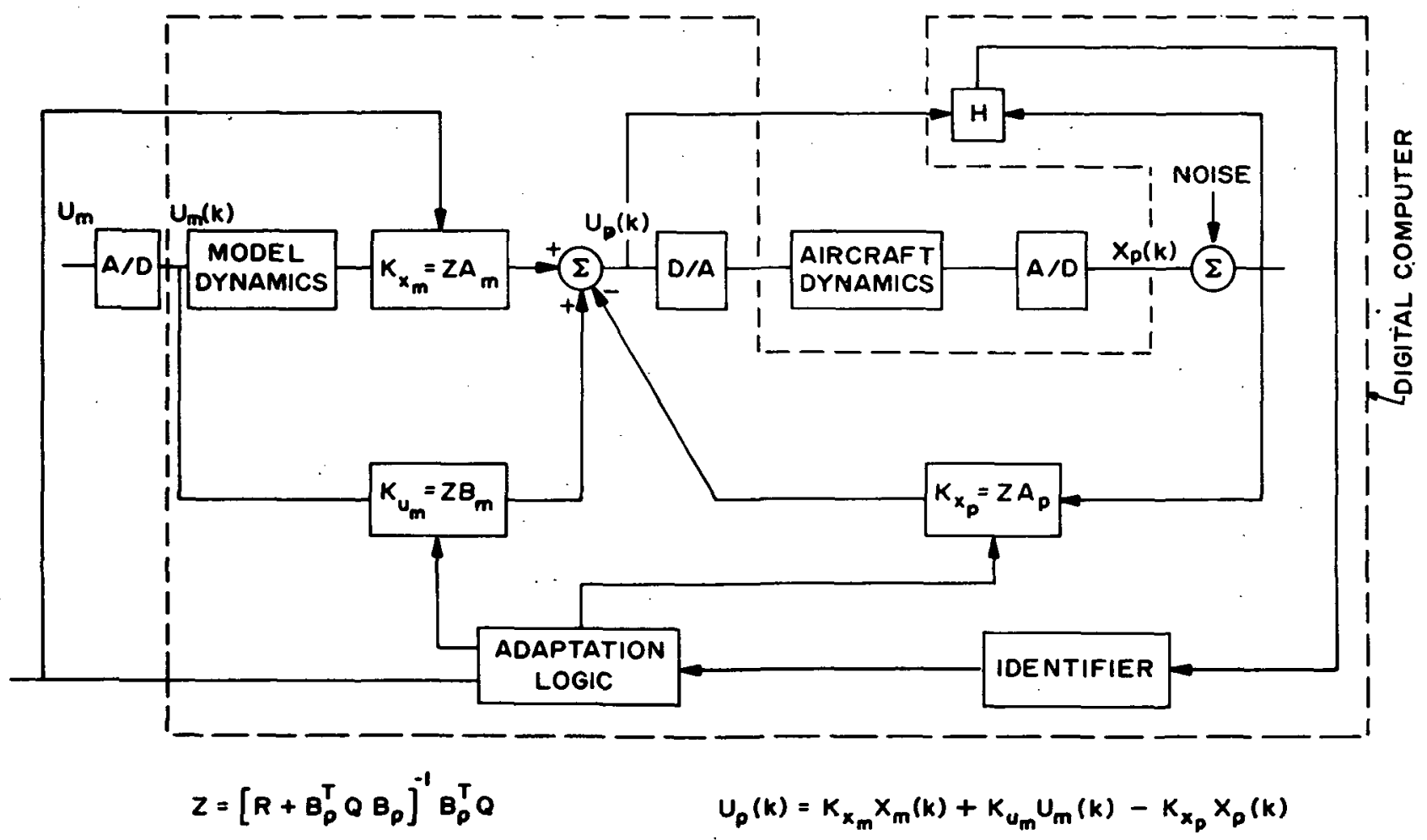

FIG. II Digital Adaptive Controller Based Upon Single Stage Optimization 
application of this controller to typical linearized lateral aircraft dymamics are discussed in 4.5.1.

One of the problems encountered in the use of same state gains was that no set of gains gave acceptable response at any FC other than that for which it was designed, particularly for a sampling period of 0.2 sec. Thus in order to maintain stability and acceptable response with a same state controller it is necessary to update the controller gains regularly at a rate determined by the fastest expected rate of change of aircraft parameters in flight.

With regard to implementation of the necessary logic for control computation, parameter estimation and gain update, the following operational procedures are required (assuming $n$ states, $m$ controls, and $l_{i}$ parameters to be identified in the $i^{\text {th }}$ state equation). CONTROL COMPUTATION

Formula:

$$
\begin{aligned}
& u_{p}(k)=K_{x_{m}} \underset{m}{x}(k)-K_{x_{p}} \underline{x}_{p}(k)+K_{u_{m}} \underline{u}_{m}(k) \\
& \underset{x m}{x}(k+1)=A_{m}{\underset{x}{m}}_{m}(k)+B_{m} \underline{u}_{m}(k)
\end{aligned}
$$

Operational Requirements

$$
\begin{aligned}
& \text { Additions: } \quad 2 m^{2}+3 m n-3 m-n+n^{2} \\
& \text { Multiplications: } n^{2}+m^{2}+3 m n
\end{aligned}
$$

\section{PARAMETER ESTTMATION}

Formula: $\quad$ eq. 2.41 
Operational Requirements :

$$
\begin{aligned}
& \text { Additions: } n(m+n)+2 l_{\text {tot }}+\sum_{i=1}^{n} l_{i}^{2}+\sum_{i=1}^{n} l_{i}^{3} \\
& \text { Multiplications: } n(m+n+1)+4 l_{\text {tot }}+\sum_{i=1}^{n} l_{i}^{2}+\sum_{i=1}^{n} l_{i}^{3} \\
& \text { where } \sum_{i=1}^{n} l_{i}=l_{\text {tot }}=\text { total number of parameters to be }
\end{aligned}
$$

\section{GAIN UPDATE}

Formula:

$$
\begin{aligned}
& \mathrm{K}_{x_{p}}=\left[R+B_{p}^{T} Q B_{p}\right]^{-1} B_{p}^{T} Q A_{p} \\
& K_{x_{m}}=\left[R+B_{p}^{T} Q B_{p}\right]^{-1} B_{p}^{T} Q A_{m} \\
& K_{u_{m}}=\left[R+B_{p}^{T} Q B_{p}\right]^{-1} B_{p}^{T} Q B_{m}
\end{aligned}
$$

Operational Requirements:

$$
\begin{aligned}
& \text { Additions: } \quad 3 m^{2}+2 m n^{2}-3 m n-2 m^{2}+m \\
& \text { Multiplications: } 4 m n^{2}+m n^{2}-2 n^{2} \\
& \text { Matrix inversions } \quad I(m \times \mathrm{m}) \text { matrix } \\
& \text { m }=1 \quad 1 \text { multiplication } \\
& \mathrm{m}=2 \quad 6 \text { multiplications and } 1 \text { addition: }
\end{aligned}
$$

As a specific example if $\mathrm{n}=4, \mathrm{~m}=2$, and $l_{1}=\ell_{3}=6$ the following total computations would be necessary. 
Control:

$\left.\begin{array}{l}38 \text { additions } \\ 44 \text { multiplications }\end{array}\right\}$ every $T_{c}$ seconds

Parameter identification:

552 additions

580 multiplications

every. $T_{i}$ seconds

12 parameter estimated

Gain computation:

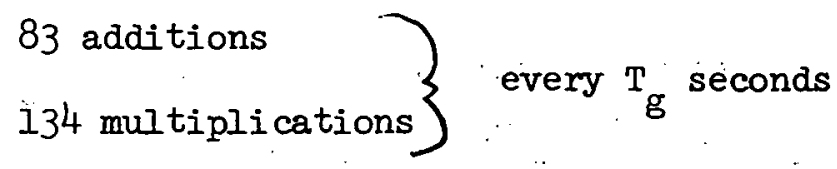

For comparison, if an extended Kalman filter had been used for simultaneous state and parameter estimation, the above 552 additions would be replaced by 2235 additions and the 580 multiplications would be replaced by 4580 multiplications.

$$
\text { For } T_{c}=T_{i}=0.2 \mathrm{sec} \text {. and } T_{g}=1.0 \mathrm{sec} \text {. the total additions and }
$$
multiplications required per second would be:

$$
\begin{array}{ll}
\text { Additions: } & 3033 \\
\text { Multiplications: } & 3254
\end{array}
$$

If only 4 parameters instead of 6 are identified in rows $I$ and 3 , and $\mathrm{T}_{c}=\mathrm{T}_{i}=0.1$ second and $\mathrm{T}_{\mathrm{g}}=1.0$ second, the computational requi rements per sec. would be:

Additions: 2043

Multiplications: 2354 


\subsection{Adaptive Logic Based on the Linear Optimal Regulator Solution}

In view of the discussion in section 2.4 .3 an adaptive controller based on the solution to the linear optimal regulator problem was chosen for implementation as shown in fig. 12. Identification was performed using the weighted least squares algorithm as defined in eq. 2.4I. Results using this adaptive logic are presented in section 4.5 .2 .

With regard to the implementation of the necessary logic for control computation, parameter estimations, and gain update, the following operational procedures are required (assuming $n$ states, $m$ controls, and $\ell_{i}$ parameters to be identified in the $i^{\text {th }}$ state equation:

CONTROL COMPUTATIONS

Formula:

$$
\begin{aligned}
& u_{p}(k+I)=K_{2 l} x_{p}(k)+\left(I+K_{22}\right) u_{p}(k)+K_{23} \cdot x_{m}(k)+K_{24} u_{m}(k) \\
& x_{m}(k+I)=A_{m} x_{m}(k)+B_{m} u_{m}(k)
\end{aligned}
$$

Operational Requirements:

$$
\text { Additions : }
$$$$
2 m^{2}+3 m n+n^{2}-m-n
$$

Multiplications: $\quad 2 m^{2}+3 m n+n^{2}$

\section{PARAMETERS ESTIMATION}

Formula:

$$
\text { Eq. } 2.41
$$

Operational Requirements:

$$
\begin{aligned}
& \text { Additions: } n(m+n)+2 l_{\text {tot }}+\sum_{i=1}^{n} l_{i}^{2}+\sum_{i=1}^{n} l_{i}^{3} \\
& \text { Multiplications: } n(m+n+1)+4 l_{\text {tot }}+\sum_{i=1}^{n} l_{i}^{2}+\sum_{i=1}^{n} l_{i}^{3}
\end{aligned}
$$




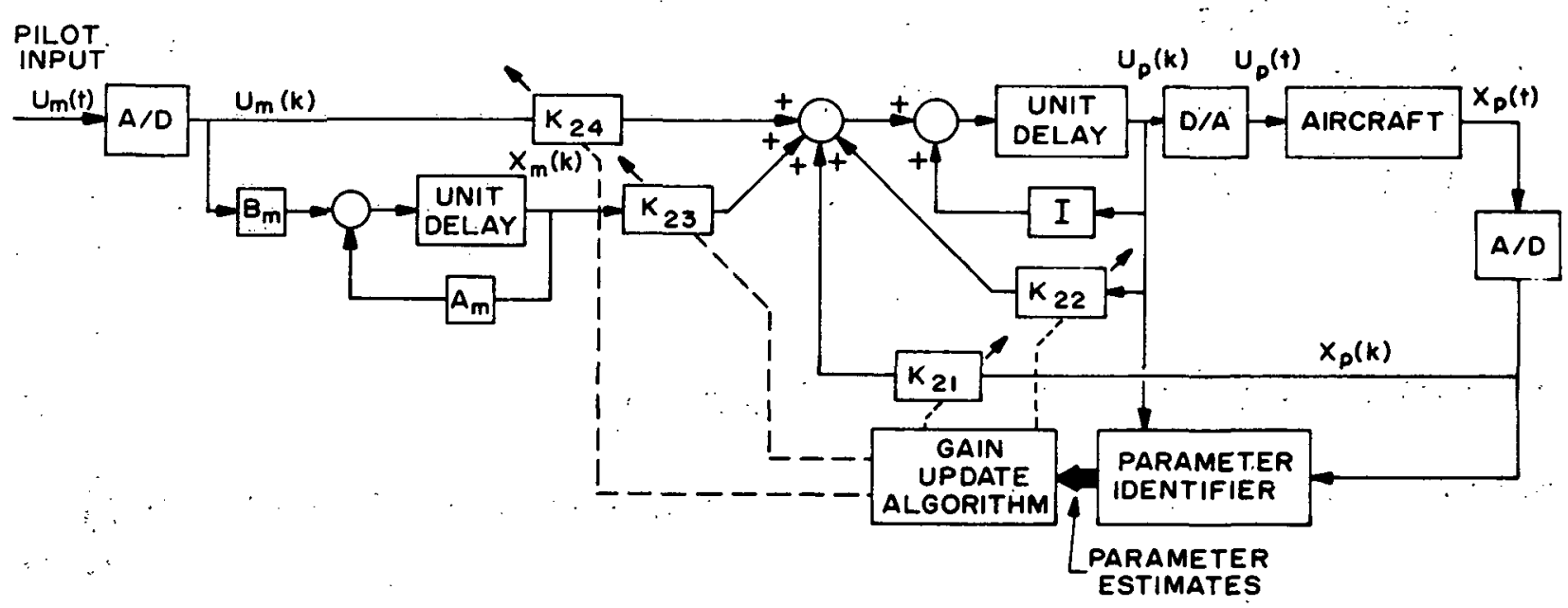

Fig..12 Digital Adaptive Controller Based on Linear Optimal Control Theory 
where $\sum_{i=1}^{n} l_{i}=l_{\text {tot }}$

\section{GAIN UPDATE}

Formula: (These were developed through partitioning of the Riccati matrix, as defined in eq. 2.31, taking into account the sparseness of the $A$ and $B$ matrices as defined by eq. 2.26).

$\mathrm{P}=\left[\begin{array}{llll}\mathrm{P} 11 & \mathrm{P} 12 & \mathrm{P} 13 & \mathrm{P} 14 \\ \mathrm{P} 12^{\mathrm{T}} & \mathrm{P} 22 & \mathrm{P} 23 & \mathrm{P} 24 \\ \mathrm{P} 13^{\mathrm{T}} & \mathrm{P}^{\mathrm{T}} & \mathrm{P} 33 & \mathrm{P} 34 \\ \mathrm{P}^{\mathrm{T}} 4^{\mathrm{T}} & \mathrm{P}^{\mathrm{P}} 4^{\mathrm{T}} & \mathrm{P} 34^{\mathrm{T}} & \mathrm{P} 44\end{array}\right]$

$$
\begin{aligned}
& P I I(k+1)=Q+A_{p}^{T}\left(P I I-P I 2(P 22+R)^{-1} P I 2^{T}\right) A_{p} \\
& P 12(K+1)=A_{p}^{T}\left[\left(P 11-P 12(P 22+R)^{-1} P 12^{T}\right) B_{p}+P 12\left(I-(P 22+R)^{-1} P 22\right)\right] \\
& \mathrm{P} 22(\mathrm{k}+\mathrm{I})=\mathrm{B}_{\mathrm{p}}^{\mathrm{T}}\left[\left(\mathrm{P} 11-\mathrm{P} 12(\mathrm{P} 22+\mathrm{R})^{-1} \mathrm{P} 12^{\mathrm{T}}\right) \mathrm{B}_{\mathrm{p}}+\mathrm{P} 12\left(\mathrm{I}-(\mathrm{P} 22+\mathrm{R})^{-1} \mathrm{P} 22\right)\right] \\
& +\left[\left(I-(P 22+R)^{-1} P 22\right) P I 2^{T}\right] B_{p}+P 22\left(I-(P 22+R)^{-I} P 22\right) \\
& K_{22}=-(P 22(k+1)+R)^{-1}\left(P 12^{T}(k+1) B_{p}+P 22(k+1)\right) \\
& \mathrm{K}_{21}=-(\mathrm{P} 22(\mathrm{k}+1)+\mathrm{R})^{-1}\left(\mathrm{Pl} 2^{\mathrm{T}}(\mathrm{k}+1) \mathrm{A}_{\mathrm{p}}\right) \\
& \mathrm{PI3}(\mathrm{k}+1)=-\mathrm{Q}+\mathrm{A}_{\mathrm{p}}^{\mathrm{T}}\left(\mathrm{P} 13-\mathrm{P} 12(\mathrm{P} 22+\mathrm{R})^{-1} \mathrm{P} 23\right) \mathrm{A}_{\mathrm{m}}
\end{aligned}
$$




$$
\begin{aligned}
P 23(k+1) & =B_{p}^{T}\left(P 13-P 12(P 22+R)^{-1} P 23\right) A_{m} \\
& +\left(P 23-P 22(P 22+R)^{-1} P 23\right) A_{m} \\
K_{23}=- & (P 22(k+1)+R)^{-1} P 23(k+1) A_{m} \\
P 14(k+1)= & A_{p}^{T}\left[P 14+\left(P 13-P 12(P 22+R)^{-1} P 23\right) B_{m}-P 12(P 22+R)^{-1} P 24\right] \\
P 24(k+1)= & B_{p}^{T}\left[P 14+\left(P 13-P 12(P 22+R)^{-1} P 23\right) B_{m}-P 12(P 22+R)^{-1} P 24\right] \\
& +\left(P 23-P 22(P 22+R)^{-1} P 23\right) B_{m} \\
& +P 24-P 22(P 22+R)^{-1} P 24 \\
K_{24}=- & (P 22(k+1)+R)^{-1}\left(P 23(k+1) B_{m}+P 24(k+1)\right)
\end{aligned}
$$

Operational Requirements: (per iteration)

$$
\text { Additions: } \begin{array}{rl} 
& 4 n^{3}+6 m^{3}+14 m^{2} n+9 n^{2} m-12 m-n^{2}-m^{2} \\
\text { Multiplications: } & 4 n^{3}+5 m^{3}+9 n^{2} m+14 m^{2} n \\
\text { Matrix inversions: } & 1(m x m) \text { matrix } \\
m=1 & 1 \text { multiplication } \\
m=2 & 6 \text { multiplications and } 1 \text { addition }
\end{array}
$$

As an example, if $n=4$ and $m=2$ the following operations are necessary:

Control:

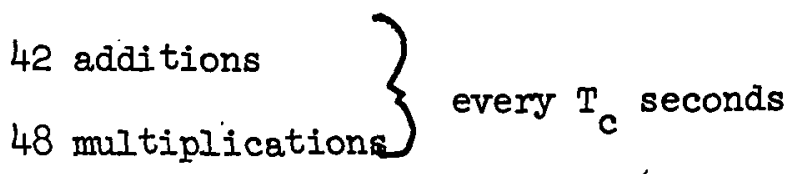


Parameter identification:

$l_{i}=(4,1,3,0)$

158 additions

178 multiplications $\left\{\right.$ every $T_{i}$ seconds

Gain computation:

$\left.\begin{array}{l}701 \text { additions } \\ 814 \text { multiplications }\end{array}\right\}$ every $\mathrm{T}_{\mathrm{g}}$ seconds

For $\mathrm{T}_{c}=\mathrm{T}_{i}=0.1 \mathrm{sec}$, and $\mathrm{T}_{\mathcal{G}}=1.0 \mathrm{sec}$. the operational requirements per second would be

Additions: $\quad 2701$

Multiplications: 3074 
4.0 RESULTS

4.1 Introduction

Evaluation of a digital adaptive flight control system requires the testing of each individual subsystem prior to integration as well as experiments on the entire system. Consequently considerable effort was expended in simulation efforts to individually evaluate each of the control algorithms discussed in Section 2.4 and each of the identification algorithms discussed in Section 2.5.

Because of the need to examine the required preciseness of identification and the degree of adaptation needed, a typical flight trajectory in the altitude-mach number plane was postulated. This is defined in Table II which cites the order and timing for a typical fighter aircraft to encounter the six given flight conditions of fig. 2l. This trajectory corresponds to an initial acceleration from Mach .3 to Mach .9 at a very low altitude, a combined climb to $3600 \mathrm{~m}$ and acceleration to Mach 1.1 , a climb to $15,000 \mathrm{~m}$, a deceleration to Mach .9 , and finally a combined dive to $6000 \mathrm{~m}$ and a deceleration to Mach .7. For simulation purposes, it was assumed that the parameters of the aircraft's discrete $A_{p}$ and $B_{p}$ matrices varied linearly with time between flight conditions.

A decision was made early in the study to use an adaptive control scheme based upon explicit identification of the unknown parameters. However, as previously mentioned, it is impractical to consider estimation of all parameters in the $A_{p}$ and $B_{p}$ matrices. A sensitivity study was therefore performed to determine those parameters which least effected system performance and which might therefore be considered constant. Results of this study are presented in Section 4.2 , and results of the identification experiments 
Table II

Flight Trajectory Used For Evaluation

\begin{tabular}{lc} 
FC & Time of encounter (seconds) \\
\hline 1 & 0 \\
2 & 30 \\
3 & 35 \\
4 & 80 \\
5 & 85 \\
6 & 120
\end{tabular}


themselves are presented in section 4.3 .

Prior to testing the adaptive loop including the identifier, an evaluation of the gain update logic itself was made under the assumption of perfect identification of various influential sets of time varying parametërs. This study, discussed in Section 4.4 led to conclusions regarding adaptation timing requirements and the number of parameters that had to be identified. Section 4.5 presents results for adaptive control incorporating online parameter estimation.

Finally, in order to assess the practicality of some of the control algorithms in a digital environment, several tests were made using NASA Langley's EAI 690 hybrid computer. Both linear and nonlinear process dymamics were considered. These results are found in Section 4.6.

4.2 State Sensitivity of Parameter Estimation Errors

4.2.1 General Procedure

The explicit calculation of the sensitivity vector

$$
\frac{\partial x_{p}(k)}{\partial q}=s(k)
$$

(where $\mathrm{q}$ is some element of the discrete aircraft matrices) was used to rank the parameters as to the need for their identification. Depending upon the size of this sensitivity, it was decided either

(1) to identify the parameter explicitly, thus having available an accurate estimate of its value, or

(2) to set that parameter to some constant value and use that constant value for all gain update work. This constant (called $q_{a v}$ ) was taken to be the average value of that parameter over all six flight conditions. Thus, the error in the value of a parameter not identified was $\Delta q=q-q_{a v}$. 
To get an approximate value for the error in the state vector due to not identifying a given parameter, the parameter error wais multiplied by a representative value for the state sensitivity to that parameter giving.

$$
\Delta x_{p}=\left(\frac{\partial x_{p}}{\partial q}\right) \Delta q
$$

Thus even if the sensitivity itself is large, a small parameter variation $\Delta q$ over the flight envelope will yield a small $\Delta x_{p}$. Each parameter was then ranked according to the error in each state of interest that would result from not identifying thịs parameter.

To calculate the sensitivity vector, a difference equation was found that determines the sensitivity vector's dynomics. ${ }^{24}$ This was done by taking the partial derivative of the state equation:

$$
x(k+1)=f(x(k), u(k), A, B)=f(k)
$$

with respect to the specified parameter, $\underline{q}$, resulting in:

$$
S(k+1)=\frac{\partial f(k)}{\partial x(k)} \quad S(k)+\frac{\partial f(k)}{\partial q}
$$

Since the sensitivity is of interest for the closed-loop system:

$$
x(x+1)=(A-B \quad K) \quad x(k)
$$

the sensitivity equations become:

$$
S(k+1)=(A-B K) S(k)+\frac{\partial(A-B K)}{\partial q} \times(k)
$$

Therefore, for a known state vector trajectory, the sensitivity of the various states to a certain parameter can be computed about that trajectory. It 
should be noted that these sensitivities will indeed be dependent upon both the system input signals $u_{m}$ and the control gains implemented.

Results of applying these sensitivity equations to the linear lateral equations of motion controlled by the single stage algorithms and the optimal regulater are next presented in sections 4.2 .2 and 4.2 .3 respectively.

\subsubsection{Sensitivity Results for Single stage Optimization}

Sensitivity calculations for the controller based upon single stage optimization were performed using a simultaneous $5^{\circ}$, aileron and $5^{\circ}$ rudder pilot command input. In general, the set of most sensitive parameters for different flight conditions were the same except for some minor differences. The set of most sensitive parameters were obtained based upon the rank ordering of the error in roll rate and sideslip. that would result if the average value of the parameters were used. It was found that in order to ensure good roll rate model following, the parameters that should be identified, in order of importance are: $B_{p}(1,2), A_{p}(1,4), B_{p}(1,1), A_{p}(1,2)$, $A_{p}(I, 3), A_{p}(I, I)$.

To ensure good sideslip following the parameters in order of importance are: $B_{p}(3,2), A_{p}(3,4), B_{p}(3,1), A_{p}(3,2), A_{p}(3,3), A_{p}(3,1)$.

These twelve distinct parameters selected for identification consist of the elements of the lst and 3 rd rows of both $A_{p}$ and $B_{p}$. Subsequent results reported in Sections 4.4 .1 and 4.5 .1 are based upon this set of parameters being identified. 


\subsubsection{Sensitivity Results for the Linear Optimal Regulator}

Sensitivity calculations for the opitmal linear regulator design were performed for a $5^{\circ}$ aileron command acting alone and for a $5^{\circ}$ ajleron command and $5^{\circ}$ rudder command applied simultaneously. It was noted that, except for minor differences, the list of most important parameters was the same for all flight conditions.

\section{For the $5^{\circ}$ aileron command, the following parameters}

(in order of descending importance) should be identified to ensure good roll rate model following:

$B_{p}(1,1), A_{p}(1,1), B_{p}(I, 2), A_{p}(3,1), A_{p}(1,2), A_{p}(3,4)$.

To achieve accurate side slip response, the following parameters should be identified:

$A_{p}(3,1), B_{p}(1,1), B_{p}(3,1), A_{p}(3,4) B_{p}(2,2)$. Note that there are eight parameters in the union of these two sets.

It is also important to note that the list of most important parameters is a function of the pilot input. For example, in comparison with the above and the results of section 4.2.2, the 12 most sensitive parameters for a $5^{\circ}$ aileron deflection and a $5^{\circ}$ rudder deflection applied simultaneously were: $A_{p}(1,1), A_{p}(1,2), A_{p}(1,3), B_{p}(1 ; 1), B_{p}(1,2)$

$$
\begin{array}{cc}
A_{p}(2,3) & B_{p}(2,2) \\
A_{p}(3,1), & A_{p}(3,2), A_{p}(3,3), \\
A_{p}(3,4), & B_{p}(3,2)
\end{array}
$$

Note that this set differs from the single stage set of 4.2 .2 in that it contains row 2 elements as well as some of the row 1 and row 3 elements. 


\subsection{Identification Results}

Evaluation of the various identification algorithms presented in Section 2.3 was performed through a series of experiments that enabled the appropriate design parameters to be tuned to the problem at hand prior to implementing the fully adaptive control system. Considerations included:

- Performance in the presence of open loop control signals having relatively large bandwidths and variances.

- Performance under open loop control signals having very little fluctuation.

- Performance under closed loop control.

- Input requirements if elements of both colums of the $B_{p}$ matrix were to be estimated. For example if $\delta_{r}=c \delta_{a}$, then the corresponding control coupling to $x_{p i}$ would be $B_{p}(i, I) \delta_{a}+B_{p}(i, 2) \delta_{r}=\left(B_{p}(i, I)+\right.$ $\left.B_{p}(i, 2) C\right) \delta_{r}$; and thus it would not be possible to specify $B_{p}(i .1)$ and $B_{p}(i, 2)$ uniquely.

- Performance as a result of assuming erroneous measurement noise characteristics. Influence of the linearization procedures discussed in Section 2.5.4.1.

In general it was observed that the simple recursive weighted least squares algorithm gave better parameter estimates than did the extended Kalman filter. Apparently the improvement to be expected in simultaneous optimization of both parameter and state estimates is more than offset by the required linearizations. Consequently in subsequent adaptive control implementations, the recursive weighted least squares algorithm was utilized. 
Comments concerming the tuning of this algorithm are contained in section 4.3.1, and examples of its performance are contained in section 4.5 . As of this writing, conclusive results were not as yet available regarding the stochastic gradient algorithm, and studies are being made of its usefulness in tracking time varying parameters in the presence of correlated noise.

Performance characteristics pertinent to the two algorithms for simultaneous state and parameter estimation, i.e., the extended Kalman filter and the separate linear state and parameter estimation algorithms (SLSPE) are contained in section 4.3.2.

4.3.1 Results Pertinent to Procedures for Parameter Estimation Only

The study conducted thus far on the stochastic gradient estimation has been basically a theoretical one and has been geared towards the development of the procedure for the identification of constant parameter multi-input multi-output systems. Further development is expected in the near future to adapt the procedure to the identification of time varying parameters and for the establishment of experimental results and analysis. This research will be concentrated on the synthesis of the correction gain $W(k)$ given in eq. 2.46, taking into account both simplicity and speed of convergence, and on the evaluation of performance in the presence of correlated noise sequences. With regard to the recursive weighted least squares algorithm, some of the results pertinent to the selection of the $P(O)$ and $Q$ matrices as used in eq. 2.41 are now presented. As stated in Section 4.1, this algorithm was selected for implementation in the fully adaptive system. 
The pertinent parameter tracking capabilities are thus discussed in section 4.5 along with the other results obtained from testing the overall system. The use of large values in the $Q$ matrix results in the ability to track rapidly varying parameters. However, it also results in a tendency to adjust parameters so as to account for variations due solely to measurement noise. Similarly, small values in the $Q$ matrix result in a more pronounced filtering of the measurement noise while the ability to track time varying parameters is degraded.

As a first approximation, the appropriate elements of $Q$ were set equal to the expected square of the appropriate parameter change per sample, and $P(0)$ wasthen set equal to the corresponding steady state solutions of eqs. 2.4lc, 2.41d, and 2.4Ie. In practice, this was accomplished by iterating through these filter equations and observing the steady state value of P. Elements of the $R$ matrix were computed in accordance with eq. 2.39, although it was observed that setting $r_{i i}$ equal to $\dot{\sigma}_{i}^{2}$ gave equally good results.

For estimation of the 12 most sensitivity parameters as used in the single stage adaptive algorithm (see section 4.2.2), the diagonal elements (all other elements were zero) of $P(O)$ and $Q$ were: 


$\begin{array}{lll}\text { parameter } & \frac{p_{i j}(0)}{A_{p}(1,1)} & \frac{q_{i 1}}{} \\ A_{p}(1,2) & 8.0 \times 10^{-4} & 4.57 \times 10^{-6} \\ A_{p}(1,3) & 2.0 \times 10^{-3} & 1.1 \times 10^{-5} \\ A_{p}(1,4) & 1.6 \times 10^{-1} & 7.75 \times 10^{-4} \\ A_{p}(3,1) & 6.5 \times 10^{-5} & 6.4 \times 10^{-10} \\ A_{p}(3,2) & 1.4 \times 10^{-5} & 4.4 \times 10^{-9} \\ A_{p}(3,3) & 2.9 \times 10^{-5} & 4.0 \times 10^{-8} \\ A_{p}(3,4) & 2.5 \times 10^{-3} & 7.1 \times 10^{-6} \\ B_{p}(1,1) & 1.4 \times 10^{-6} & 4.4 \times 10^{-9} \\ B_{p}(1,2) & 1.9 \times 10^{-2} & 9.4 \times 10^{-5} \\ B_{p}(3,1) & 4.4 \times 10^{-3} & 6.4 \times 10^{-5} \\ B_{p}(3,2) & 3.8 \times 10^{-4} & 1.0 \times 10^{-6}\end{array}$

For the eight most sensitive parameters as used in the adaptive controllex based upon optimal regulator logic (see Section 4.2.3) the diagonal elements of $P(O)$ and $Q$ were:

$\begin{array}{lcc}\text { Parameter } & \frac{P_{i j}(0)}{A_{p}(1,1)} & \frac{Q_{i j}}{6.0 \times 10^{-5}} \\ A_{p}(1,2) & 2.0 \times 10^{-3} & 1.6 \times 10^{-7} \\ B_{p}(1,1) & 2.0 \times 10^{-3} & 3.4 \times 10^{-4} \\ B_{p}(1,2) & 1.2 \times 10^{-2} & 2.3 \times 10^{-4} \\ B_{p}(2,2) & 5.0 \times 10^{-2} & 3.4 \times 10^{-6} \\ A_{p}(3,1) & 5.0 \times 10^{-3} & 8.6 \times 10^{-10} \\ A_{p}(3,4) & 1.2 \times 10^{-5} & 1.2 \times 10^{-7} \\ B_{p}(3,1) & 1.5 \times 10^{-5} & 2.0 \times 10^{-6}\end{array}$


4.3.2 Results pertinent to Estimation of Both States and Parameters Conclusions pertinent to the performance characteristics of

both the Kalman filter and the SLSPE were based upon simulation on an IBM $360 / 50$ digital computer. Sampling periods of $1 / 30$ second and $1 / 10$ second were incorporated, and estimation of the four lateral states and the eight most sensitive parameters for the linear regulator logic. (as presented in section 4.2.3) was considered. Unless otherwise cited, measurement noise was simulated as uncorrelated processes having the variances given in Table I.

\subsubsection{Kalman Filter Results}

The following conclusions were reached regarding the performance of the extended Kalman filter:

Selection of initial covariance matrix $\mathrm{P}(0)$ Assuming perfect knowledge of the initial state, the corresponding elements of $P(0)$ should be zero. The terms of $P(0)$ corresponding to the parameters can be zero if fictitious noise with a covariance matrix $Q$ is used to model the parameter variation; otherwise these elements should be proportional to the expected square of the per sample change in the parameter. Measurement noise covariance matrix It was noted that the use of the exact noise covariance in the filter equations was not necessary for convergence. It was observed that convergence was possible with the noise covariance $R$ set five times larger than 
the actual noise covariance matrix; however convergence did not occur for $R$ matrix elements much lower than those in the actual measurement noise covariance matrix. Nominal trajectory selection

For time varying parameters it was found that the best. procedure for updating the nominal trajectory was through the use of the most recent state and parameter estimates for defining the point about which Iinearization was to be performed. This is Procedure 4 as discussed in Section 2.5.4.1. For constant: parameters, it was found that the best trajectory to Iinearize about was defined by Procedure 2 . Feding memory implementation From experimental results, it was observed in general that the use of a fictitious noise input for modeling the parameter variations yielded better convergence properties than did the procedure using an experimental weighting factor. This is illustrated in fig. 13 for estimation of $a_{31}$ under open-loop excitation $\left( \pm 30^{\circ}\right.$ rudder at $1.5 \mathrm{~Hz}$ and $\pm 30^{\circ}$ aileron at $1.0 \mathrm{~Hz})$. State measurement noise was uncorrelated with variances of one tenth those listed in Table I. 


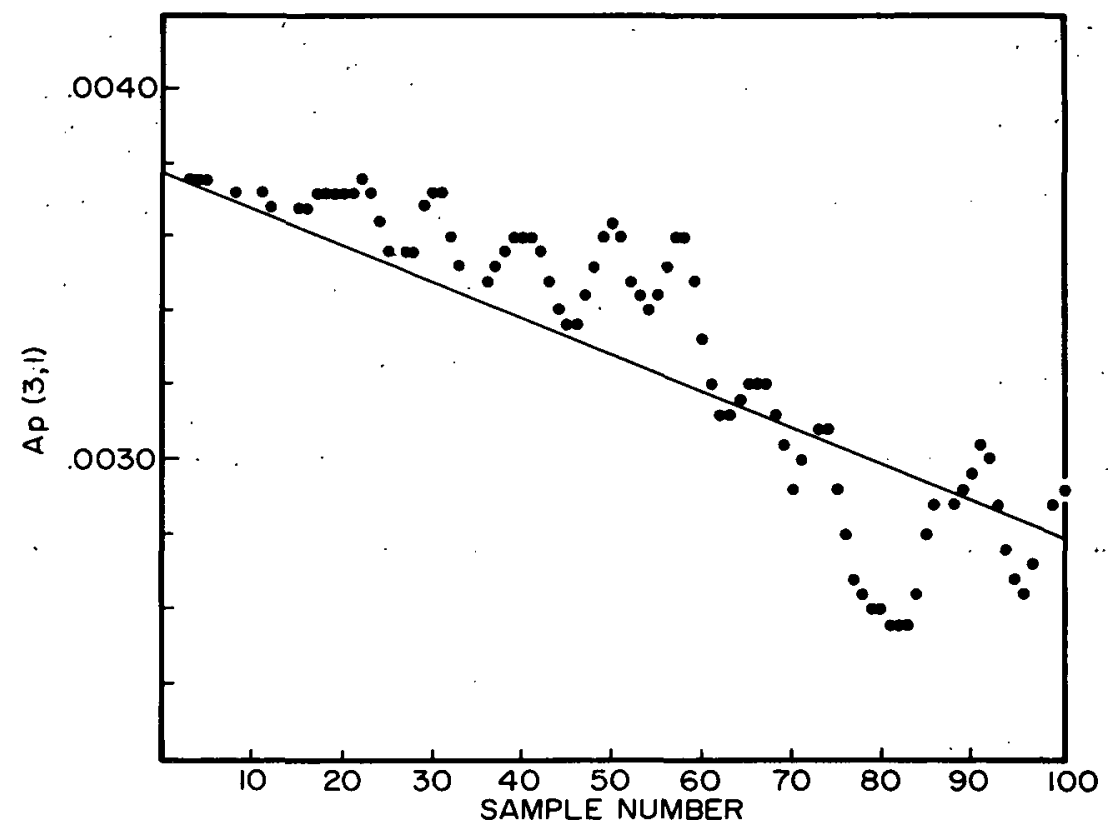

FIG. 13 a TYPICAL PARAMETER TRACKING USING FICTITIOUS NOISE TO MODEL PARAMETER VARIATIONS

- - ESTIMATES tRUE VALUes

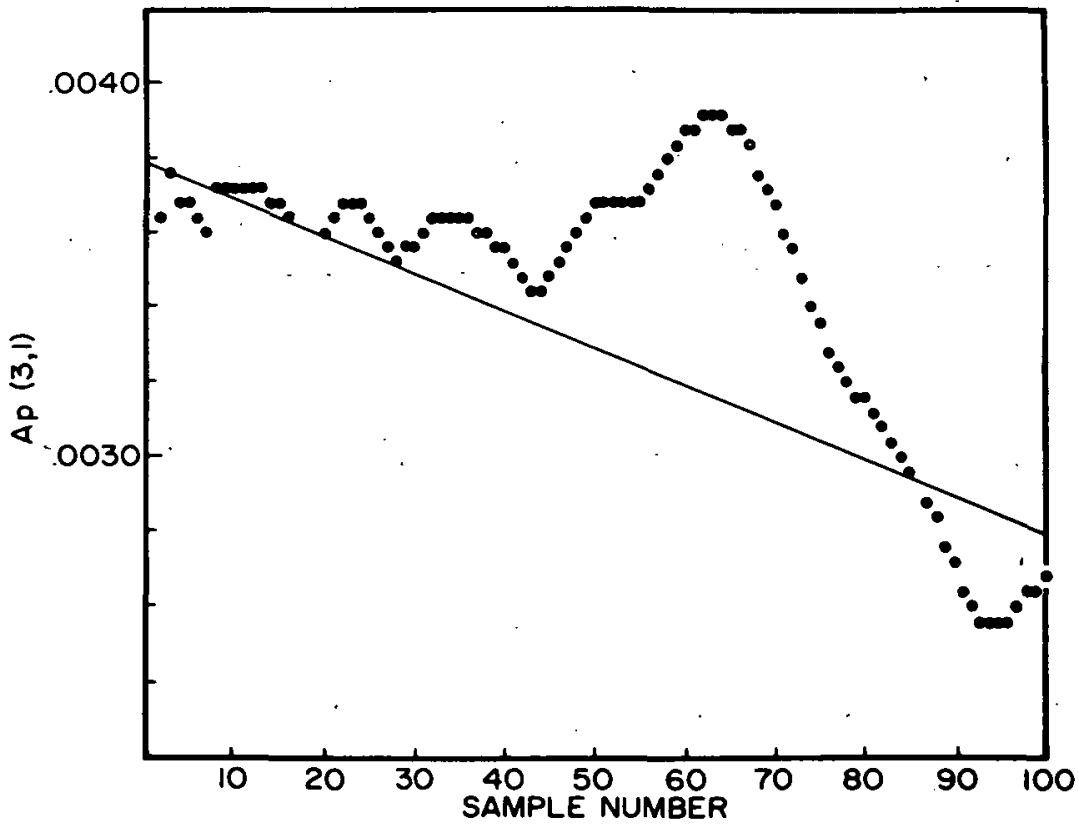

FIG. 13 b TYPICAL PARAMETER TRACKING USING A FADING FACTOR IN THE PERFORMANCE INDEX 


\subsubsection{SLSPE Results}

In using the SLSPE it was observed that the parameters estimates were biased and that convergence was poor if parameters in both columns of the $B_{p}$ matrix were identified. However, these results corresponded to experiments in which a recursive step of both the parameter estimation algorithm and the state estimation algorithm were performed at each and every sample. In view of the results obtained using the weighted least squares algorithm for parameter estimation only, it is recommended that either state estimation not be performed as often as the parameter estimation or that the state measurements, not the state estimates, be used in the parameter estimation algorithm. Future efforts will consider these items.

\subsection{Performance Under Perfect Identification Prior to Interfacing Adaptive Control Logic}

With an online identifier, it is necessary to validate the results of the sensitivity study described in 4.2.1 and to determing how often adaptation should be performed. Upper bounds to these figures can be obtained by simulating an adaptive controller with a perfect identifier. Thus while parameters not being "identified" would be assumed at their average values, true values would be used for these parameters designated for identification.

\subsubsection{Performance of the Single Stage Algorithm}

The following two experiments pertain to the performance of

the single stage controller:

Experiment I

Objective: Assuming no noise and perfect identification of all parameters, determine suitable gain update periods. 
Procedure: At each gain update sample time, the gains optimal for the current flight conditions were to be computed from eq. 2.19 and held constant until the next gain update sample time. These gains at the $6 \mathrm{FC}$ 's are given in Appendix B.

Constant factors:

$$
\text { Pilot input: } \pm 5^{\circ} \text { aileron, } 0.1 \mathrm{hz} \text { square wave }
$$

Variable factors:

$$
\begin{array}{ll}
\text { Control sampling periods: } & .2, .033 \text { sec. } \\
\text { Gain update periods: } & 1.0,2.5,5.0 \text { sec. }
\end{array}
$$

Results: Figure 14 indicates good model following for a gain update period of $2.5 \mathrm{sec}$. and a control period of $0.2 \mathrm{sec}$. A gain update period of $5.0 \mathrm{sec}$. was found to be satisfactory for a control sampling interval of $.033 \mathrm{sec}$, but unsatisfactory for a control period of $0.2 \mathrm{sec}$.

\section{Experiment II}

Objective: Assuming that only selected sets of 10-12 parameters are to be identified perfectly, with the remainder set equal to their average value over the typical flight trajectory for six FC's determine the necessary frequency of gain updating. The parameter sets to be identified are those deemed to be the most influential as discussed in 4.2.1.

Procedure: At each gain update sample, the gains were to be computed from eq. 2.19 using the true values for those parameters being "identified" and average (over the 6. FC's) values for these parameters not being identified.

Constant factors:

$$
\begin{aligned}
& \text { Pilot input: } \pm 5^{\circ} \text { aileron, } 0.1 \mathrm{hz} \text { square wave } \\
& \text { Control sample period: } 0.2 \mathrm{sec} .
\end{aligned}
$$




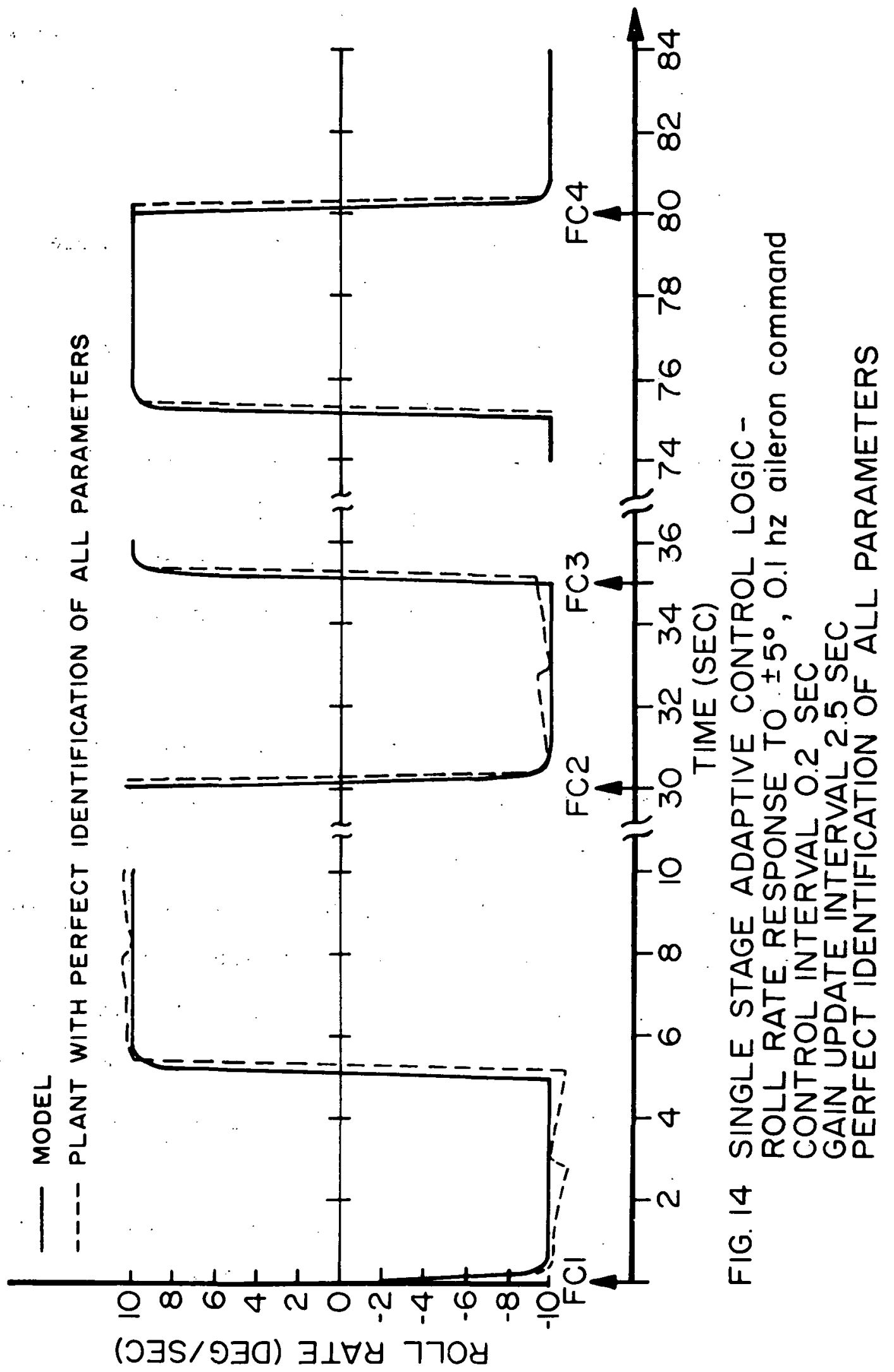


Variable factors:

Parameters identified: 10 and 12 most influential parameters. Gain update periods: $1.0,2.5 \mathrm{sec}$.

Results: Figures 15 and 16 indicate the significant improvement that results from identifying 12 rather than 10 parameters especially between FC3 and FC4. This is true for both gain update periods. The most notable improvement in using a gain update period of 1.0 rather than 2.5 sec. is most evident between FCI and FC2.

Conclusions of both experiments: For the overall adaptive implementation, identify the 12 most influential parameters, and based upon these, update gains every $1.0 \mathrm{sec}$.

\subsubsection{Performance of the Linear Optimal Regulator \\ The following three experiments pertain to the performance} of the linear optimal regulator design:

\section{Experiment I}

Objective: Assuming no noise and perfect identification of all parameters, determine suitable gain update periods.

Procedure: At each gain update sample time, the $P$ matrix is recomputed from a single iteration of the Riccati eq. (2.31) using those values of $A_{p}$ and $B_{p}$ which correspond to the present flight conditions. Control gains are then computed from eq. 2.29. (See Appendix B for the gains corresponding to the 6 given FC's).

Constant factors:

$$
\text { Pilot input: } \pm 5^{\circ} \text { aileron, } .2 \mathrm{hz} \text { square }
$$




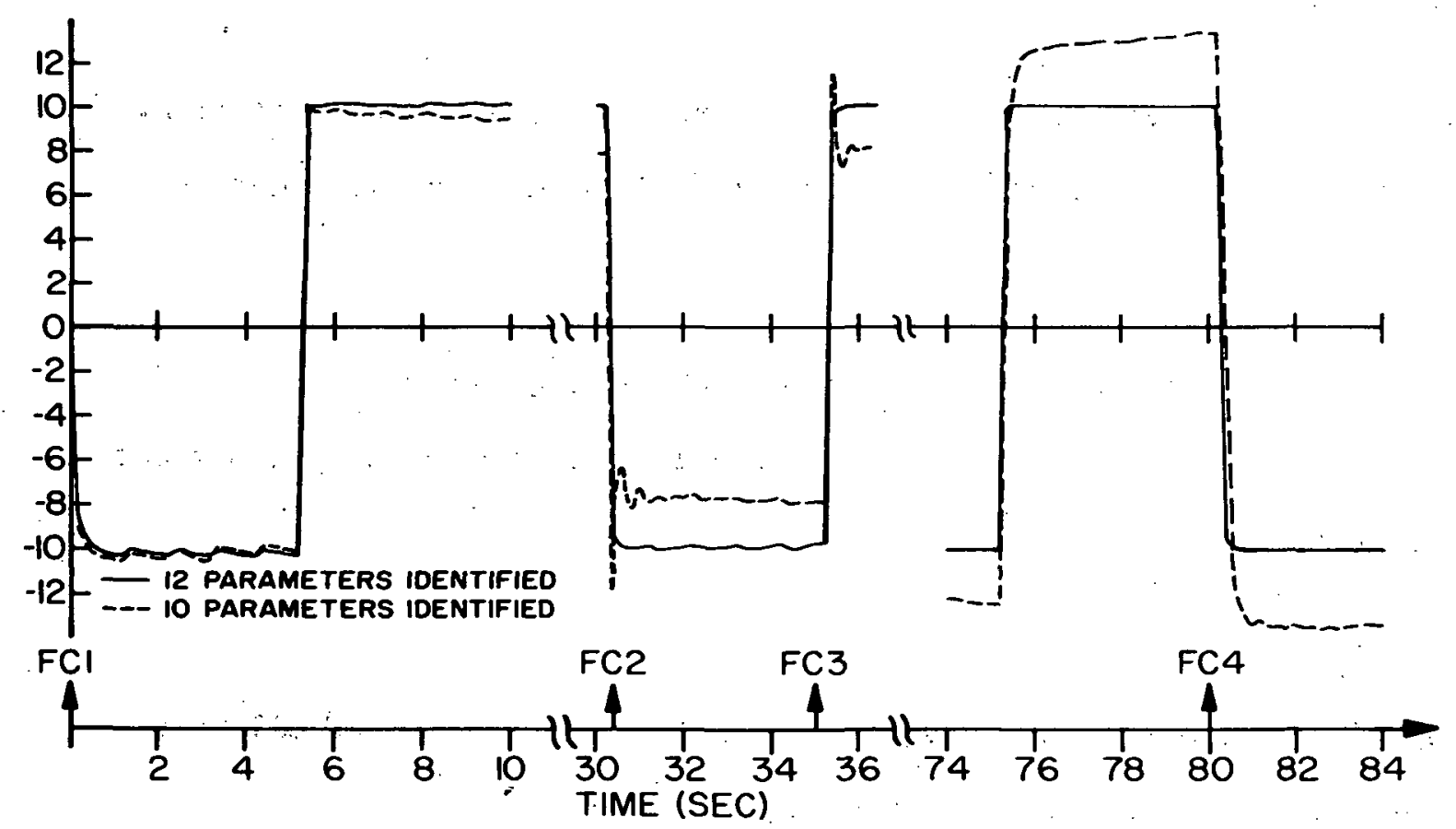

FIG. 15 SINGLE STAGE ADAPTIVE CONTROL LOGIC-ROLL RATE RESPONSE CONTROL INTERVAL 0.2 SEC

GAIN UPDATE INTERVAL IO SEC

PERFECT IDENTIFICATION OF IO AND 12 PARAMETERS 


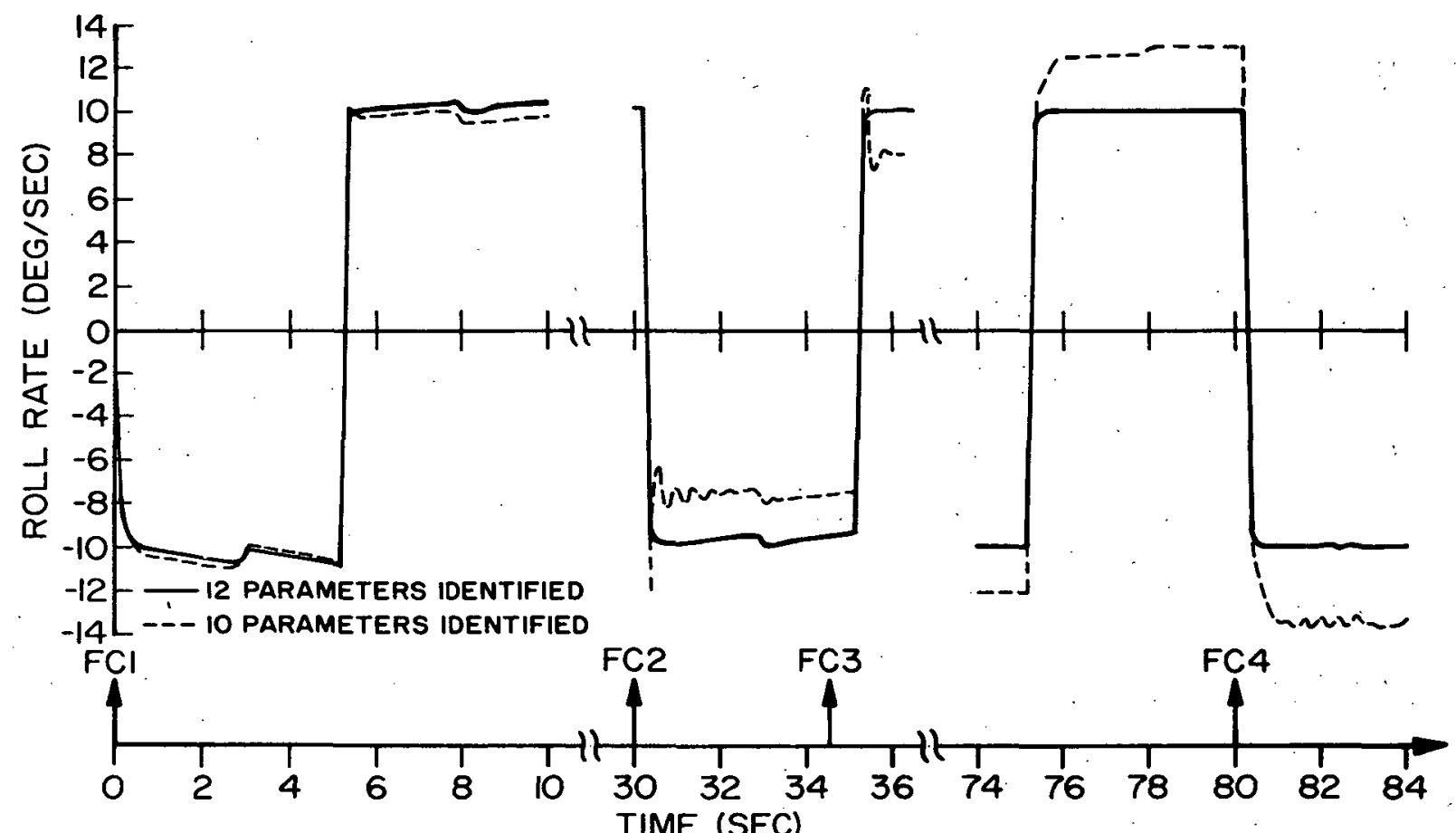

FIG. 16 SINGLE STAGE ADAPTIVE CONTROL LOGIC-ROLL RATE RESPONSE CONTROL INTERVAL O.2 SEC

GAIN UPDATE INTERVAL 2.5 SEC

PERFECT IDENTIFICATION OF 10 AND 12 PARAMETERS 
Control sample period: 0.1 sec.

Variable factors:

Gain update periods: 1, 5, $10 \mathrm{sec}$

Results: Figures $17 a$ and $b$ show good model following for a gain update period of 1.0 sec., while figs. 18a and $b$ indicate some degradation in performance for a gain update period of $5.0 \mathrm{sec}$. Stability for the closed loop system was observed for the three tested gain update periods of 1 , 5 and $10 \mathrm{sec}$.

Experiment II

Objective: Under the assumption of no noise and perfect identification of the 8 parameters, chosen by the sensitivity method described in section 4.2 , with all others set to their average value over all 6 flight conditions, determine the required frequency of gain update.

Procedure: At each gain update sample, the gains were to be computed from one iteration of the Riccati eq. (2.3I) using the true values for the eight parameters being identified and average (over the 6 FC's) values for the remaining 16 parameters.

Constant factors:

Pilot input: $\pm 5^{\circ}$ aileron, $.2 \mathrm{hz}$ square wave Control sample period: 0.1 sec.

Variable factors:

Gain update periods: 1, 5, $10 \mathrm{sec}$.

Results: Figs. 19 and 20 1llustrate the improvement in model following which results from using a 1 sec. gain update period rather than a 5 sec. period. For a 10 sec. period, the stability of the closed loop system was marginal. 


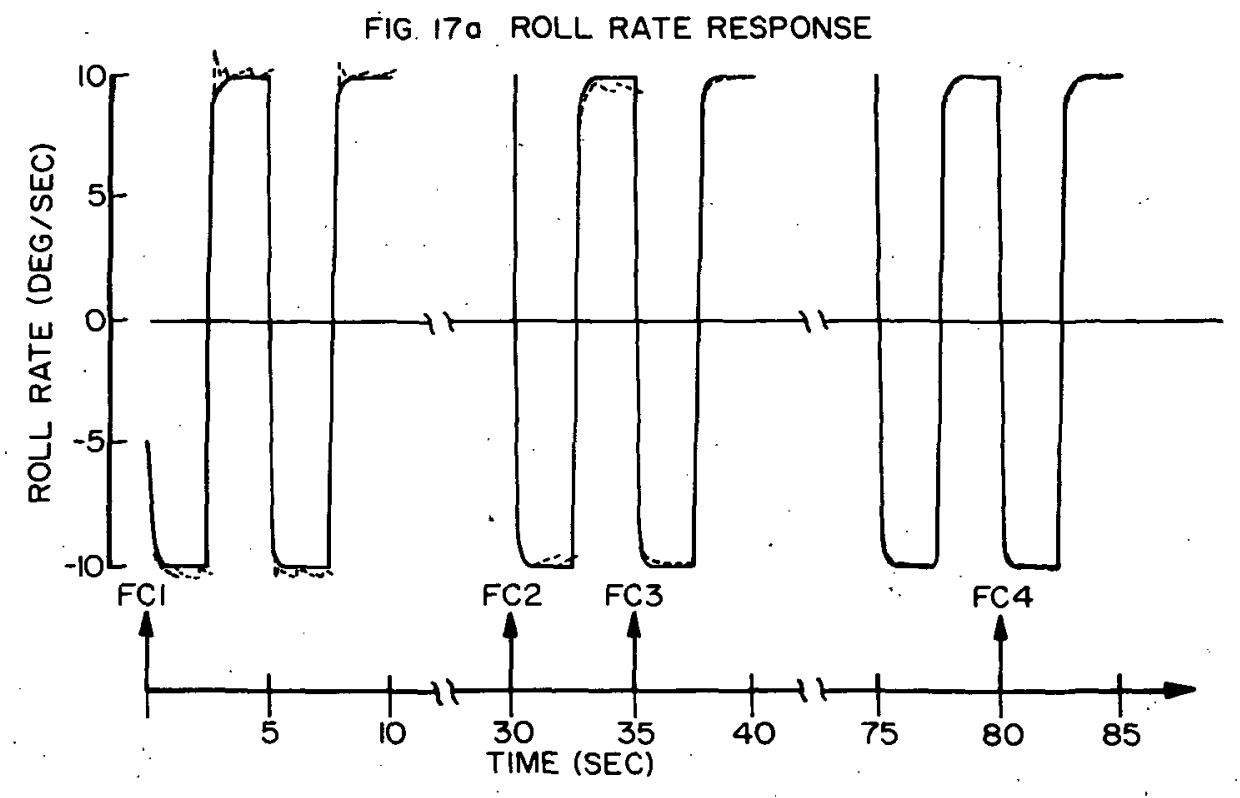

CONTROL INTERVAL O.I SEC

GAIN UPDATE INTERVAL IO SEC

PERFECT IDENTIFICATION OF ALL PARAMETERS

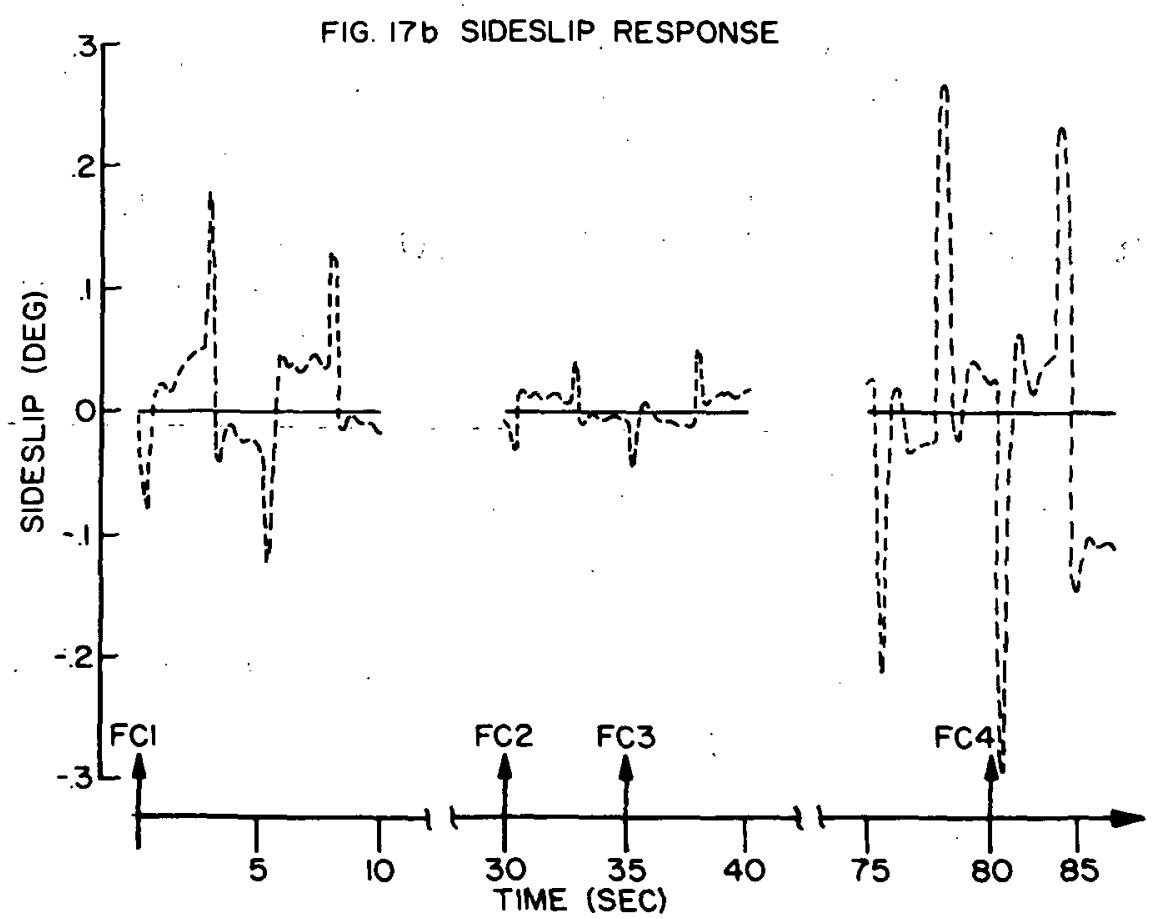

FIG. 17 LINEAR REGULATOR ADAPTIVE CONTROL LOGICROLL RATE AND SIDESLIP RESPONSES TO 


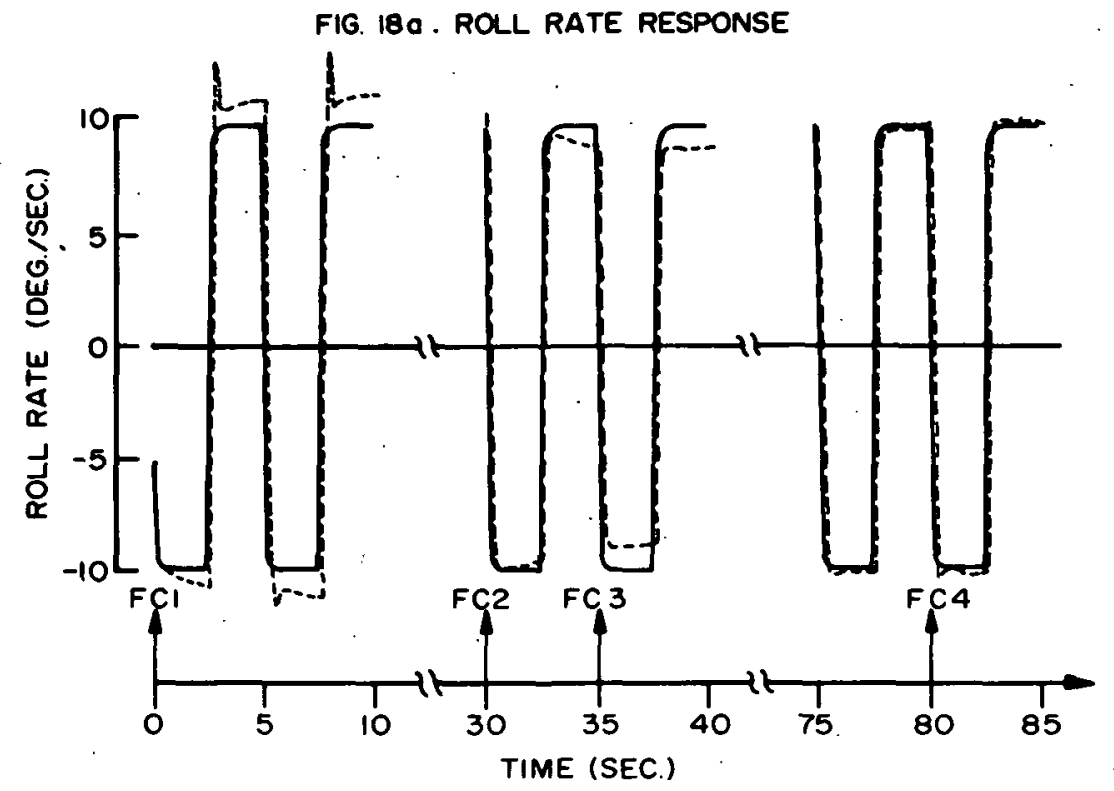

CONTROL INTERVAL O.I SEC.
GAIN UPDATE INTERVAL 5.O SEC.
PERFECT IDENTIFICATION OF
ALL PARAMETERS
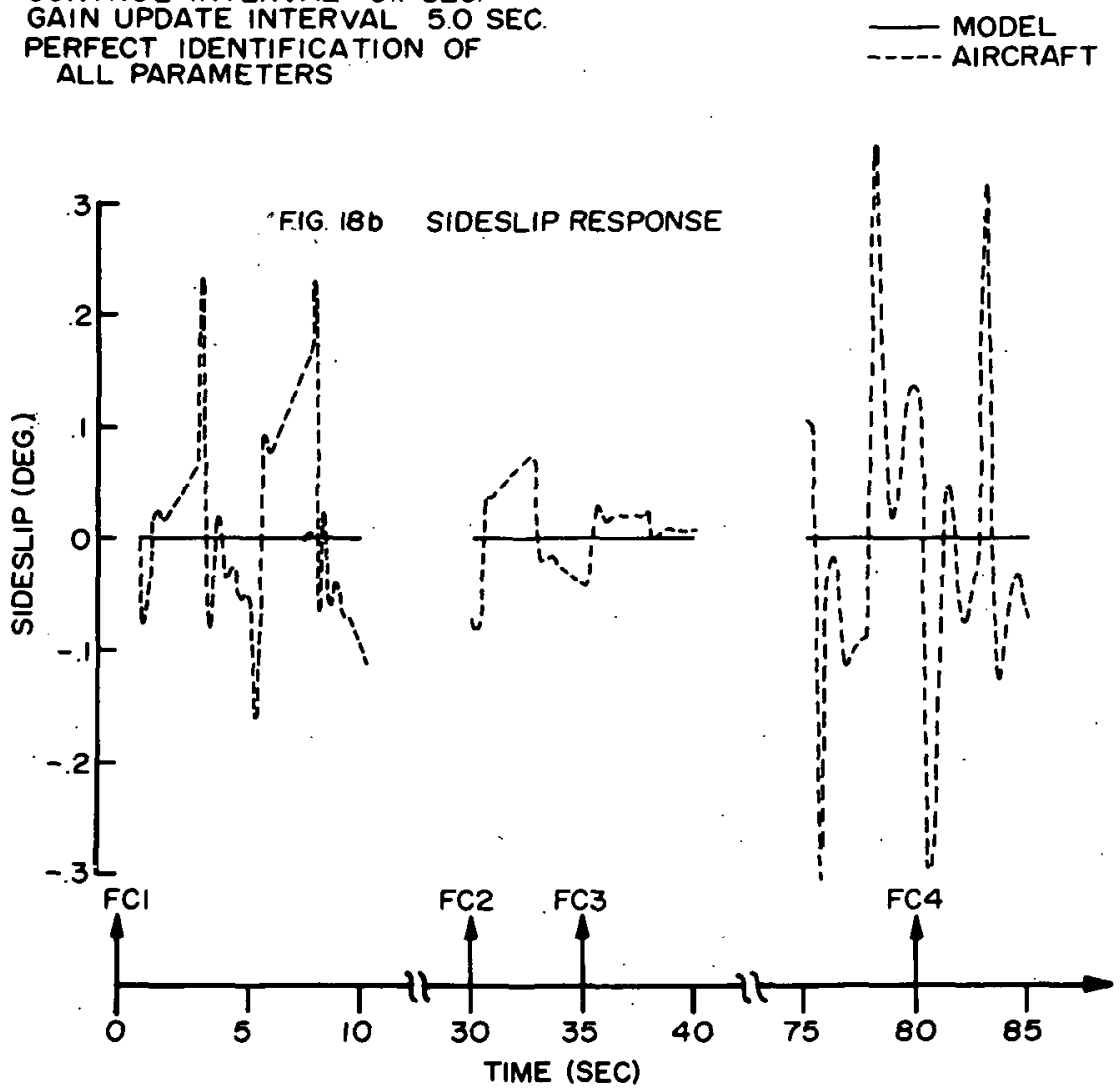

FIG. 18 LINEAR REGULATOR ADAPTIVE CONTROL LOGICROLL RATE AND SIDESLIP RESPONSES TO $\pm 5^{\circ}, 0.2 \mathrm{hz}$ AILERON COMMAND 


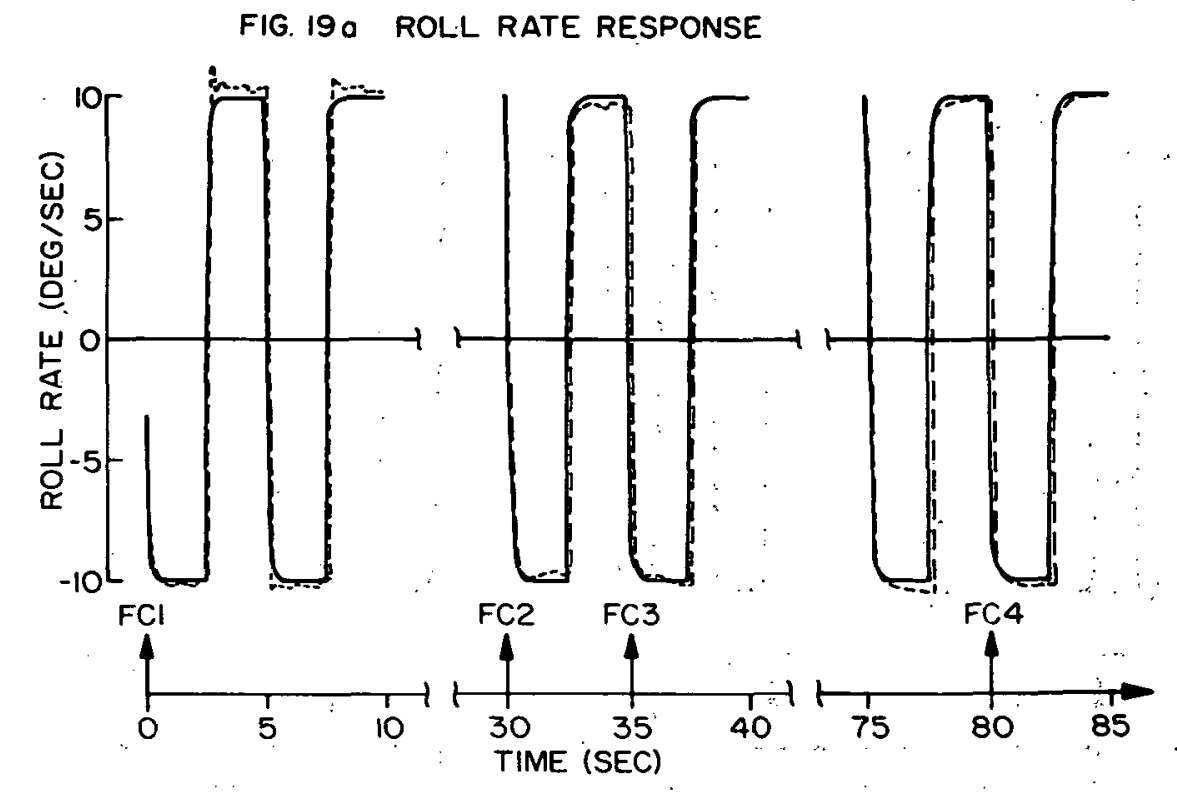

CONTROL INTERVAL O.I SEC

GAIN UPDATE INTERVAL 1.0 SEC

PERFECT IDENTIFICATION OF EIGHT PARAMETERS

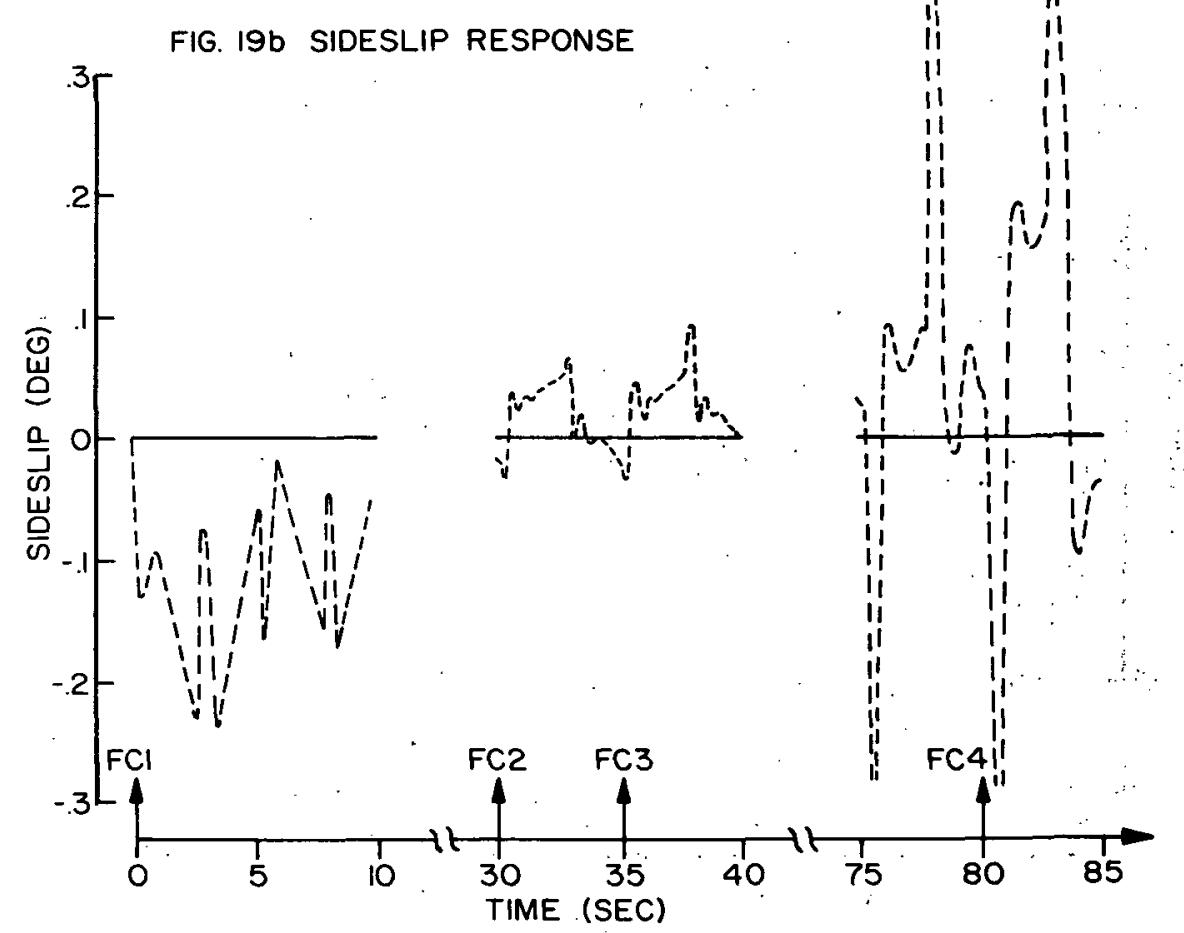

FIG. 19 LINEAR REGULATOR ADAPTIVE CONTROL LOGIC ROLL RATE AND SIDESLIP RESPONSES TO $\pm 5^{\circ}, 0.2 \mathrm{hz}$ AILERON COMMAND 


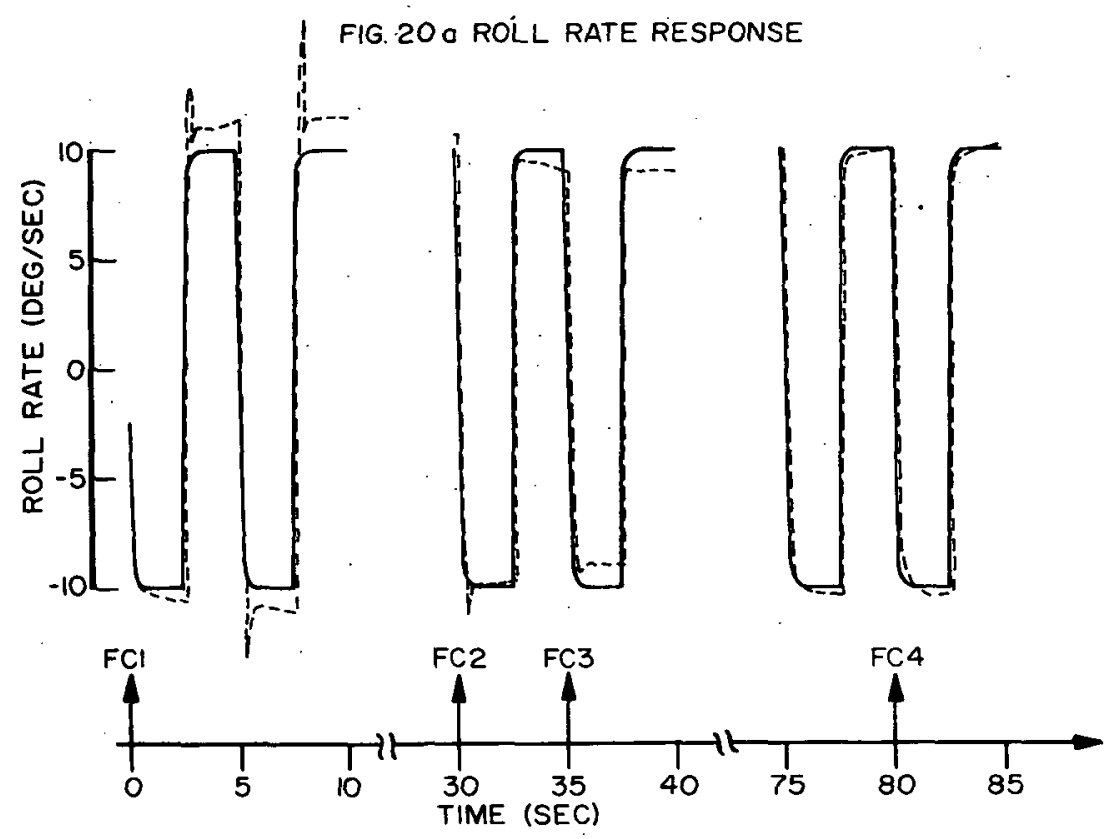

CONTROL INTERVAL 0.1 SEC

GAIN UPDATE INTERVAL 5.0 SEC

PERFECT IDENTIFICATION OF EIGHT PARAMETERS

FIG. 2Ob SIDESLIP RESPONSE

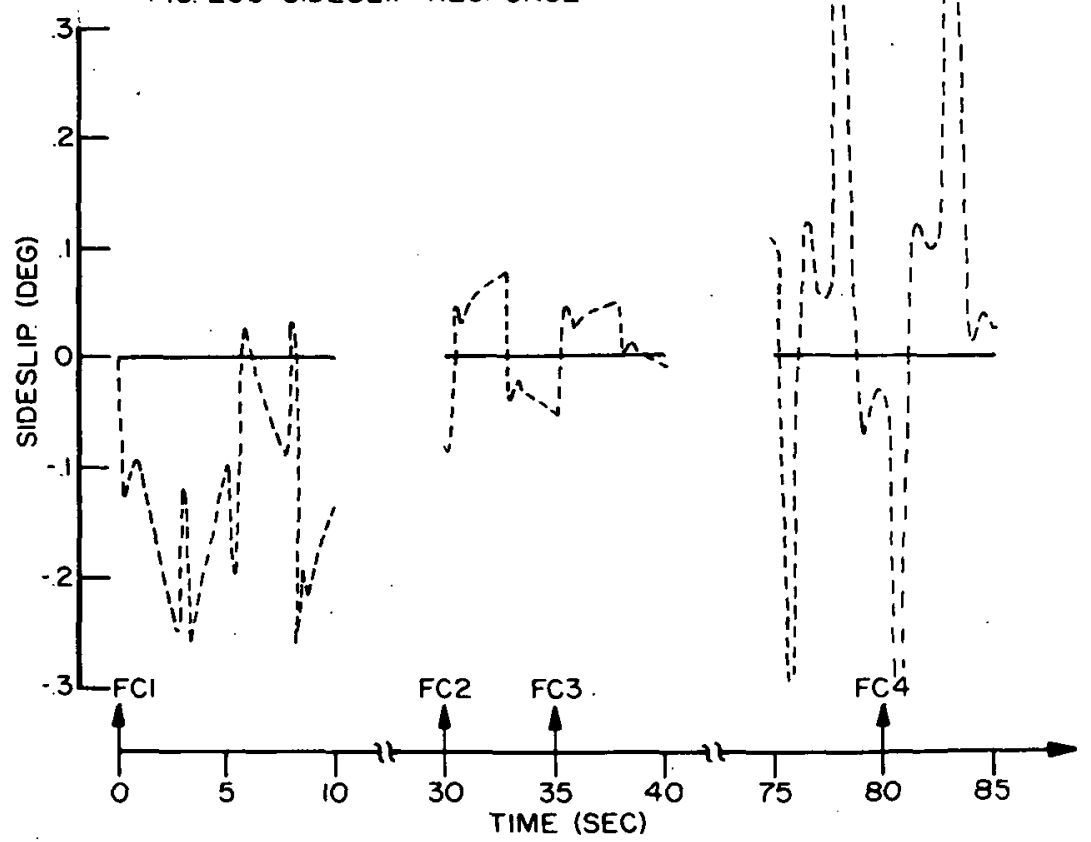

FIG. 20 LINEAR REGULATOR ADAPTIVE CONTROL LOGICROLL . RATE AND SIDESLIP RESPONSES TO $\pm 5^{\circ}$, O.2 hz AILERON COMMAND 
Note that relative to the results of Experiment $I$, there is more deterioration of side slip model following than there is of roll rate following. This is true because the set of eight parameters identified is more influential in defining the roll rate dynamics.

Experiment III

Objective: Determine how the results of Experiment II are affected if noisy state measurements are used for control computation.

Procedure: Same as for Experiment II except control signals were to be computed from products of the gains and noisy state measurements.

Constant factors:

Pilot input: $\pm 5^{\circ}$ aileron, $.2 \mathrm{hz}$ square wave

Control semple period: 0.1 sec.

Gain update periods: 1, 5 secs.

Variable factors:

Noise statistics: Table I characteristics achieved using first order filters with breaks at the given bandwidths. RMS values given in Table $I$ and $I / 10$ these values were used.

Results: Figure 21 shows that feeding back states contaminated with noise results in severe. jittering of the aircraft response... Some sort of prefiltering is thus necessary. As expected, lowering the rms values of the noise samples reduced the magnitude of this jitter.

Conclusions from all three experiments: For implementation of an overall adaptive system, identify the eight most influential parameters discussed in Section 4.2.3, and using these, update the gains every one to five seconds. 


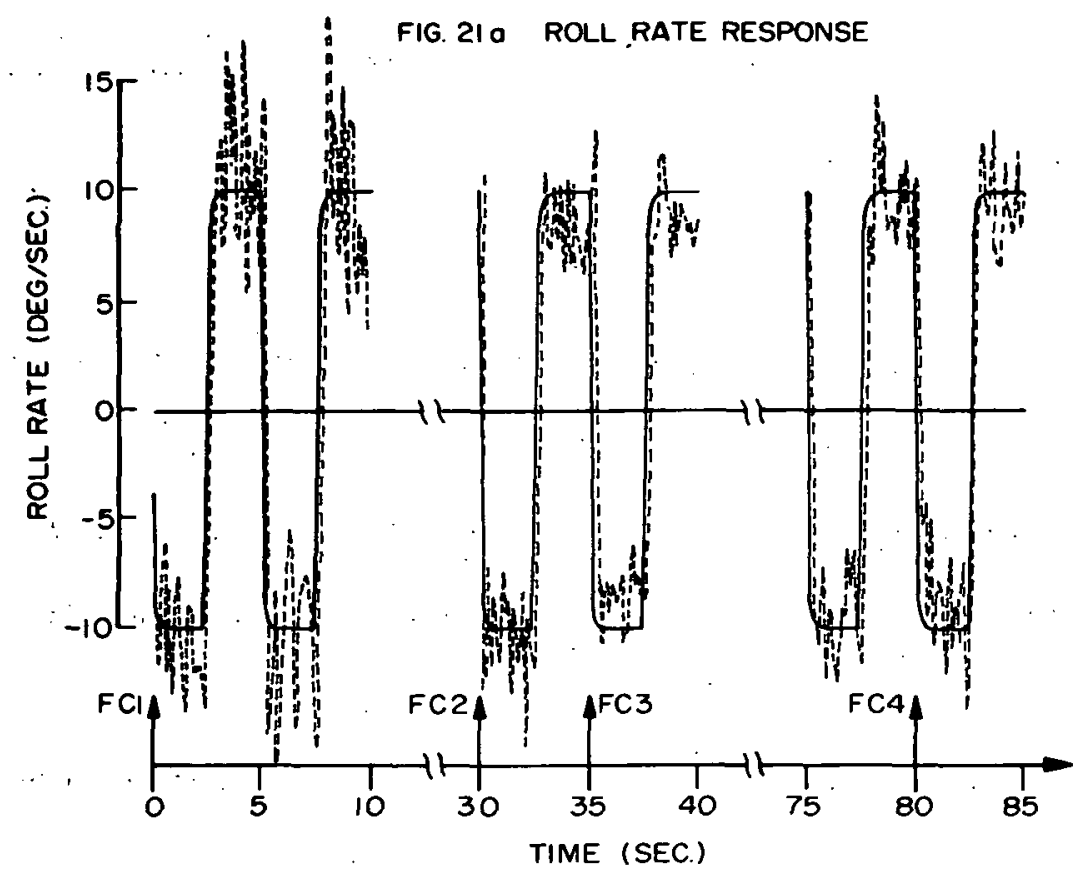

CONTROL INTERVAL O.I SEC

GAIN UPDATE INTERVAL 5.0 SEC.

PERFECT IOENTIFICATION OF EIGHT PARAMETERS

- MODEL

FEEDBACK OF ACTUAL MEASUREMENTS

--- AIRCRAFT

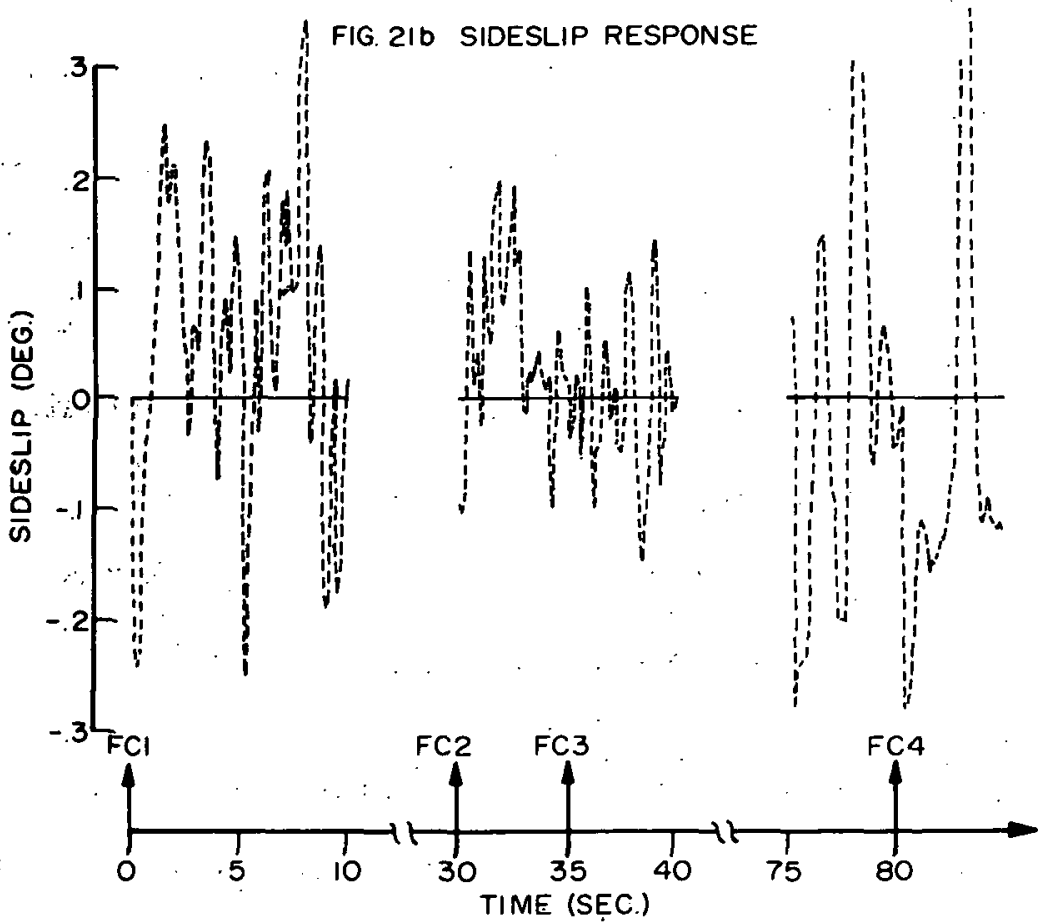

FIG. 21 LINEAR REGULATOR ADAPTIVE CONTROL LOGICROLL RATE AND SIDESLIP RESPONSES TO $\pm 5^{\circ}, 0.2 \mathrm{hz}$ AILERON COMMAND 
Prefiltering is needed for state measurements contaminated by noise having the characteristics of Table I.

4.5 Adaptive Control System Performance Evaluation 4.5.1 Adaptive Control Loop Fxperiment - Single -Stage Optimization Procedure

Objective: To study the behavior of the adaptive control system designed by coupling a weighted least squares identifier with the single stage controller logic as shown in fig. Il.

Procedure: Using noisy state measurements with no prefiltering, parameter estimates were obtained at each control sample period and then used at each gain update period to compute the control gains according to eq. 2.19. The noisy state measurements were then used with these gains for control computation.

Design factors:

Pilot input: $\pm 5^{\circ}$ aileron, $0.1 \mathrm{hz}$ square wave. Control sample period: $0.2 \mathrm{sec}$. Gain adaptation period: $1.0 \mathrm{sec}$. Parameters identified: $A_{p}(1,1), A_{p}(1,2), A_{p}(1,3), A_{p}(1,4)$, $A_{p}(3,1), A_{p}(3,2), A_{p}(3,3), A_{p}(3,4)$,
$B_{p}(1,1), B_{p}(1,2), B_{p}(3,1), B_{p}(3,2)$.

Remaining parameters: Set at the average values computed over the 6 given FC's.

Measurement noise: As defined in Table I. 
Results:

\section{Responses}

Figure 22 shows the roll rate response for portions of the test flight trajectory. The noisy state feedback results in severe jittering of the aircraft and thus relatively poor model following particularly at FC's 1 and 2. Side slip was generally limited to $\pm 0.4^{\circ}$ except for FCl where the maximum excursion was $2.8^{\circ}$.

Parameter tracking

Figures 23a, b, c, d illustrate parameter tracking behavior between FCl and FC2 during which model following is the worst. To be noted is that despite the existence of a bias in the estimate, the general trend in the variation is followed.

Control gains

Comparisons between the optimal and adapted control gains are given in figs. 24a, b, c, d for part of the interval between FCl and FC2. With the exception of the roll rate feedback gains, the adapted gains were relatively close to the optimal gains. In fact, beyond the 20 second interval depicted in fig. 24, all gains converged closer to the true values.

Conclusions: In view of the fact that the adaptation procedure was capable of producing reasonable feedback gains, it is concluded that a workable system should result from the inclusion of prefilters for removing jitter and the usage of an unbiased parameter identification algorithm. 


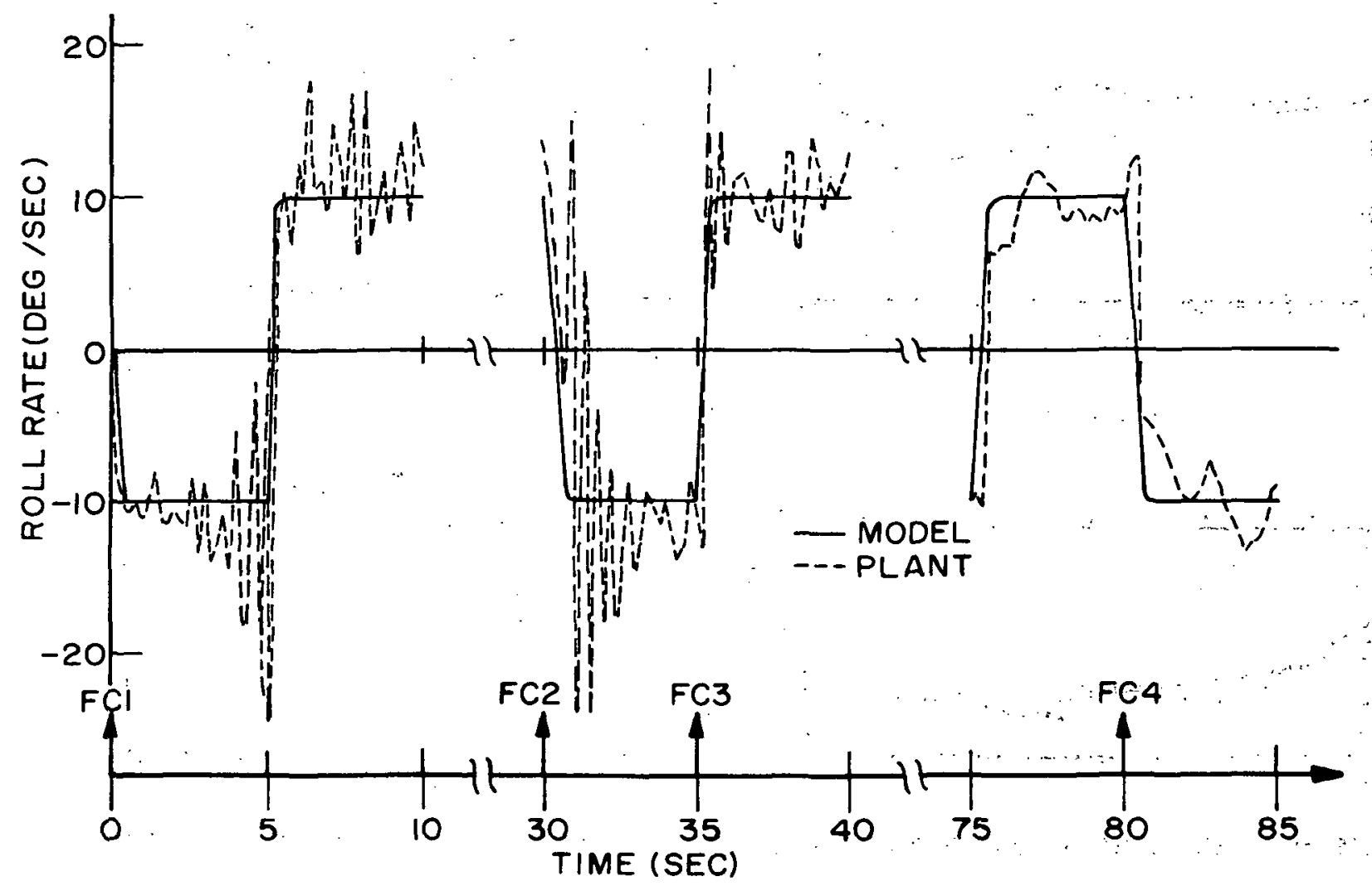

FIG. 22 ROLL RATE RESPONSE, SINGLE STAGE LOGIC WEIGHTED LEAST SQUARES IDENTIFICATION OF TWELVE PARAMETERS CONTROL INTERVAL 0.2 SEC GAIN UPDATE 1.0 SEC 

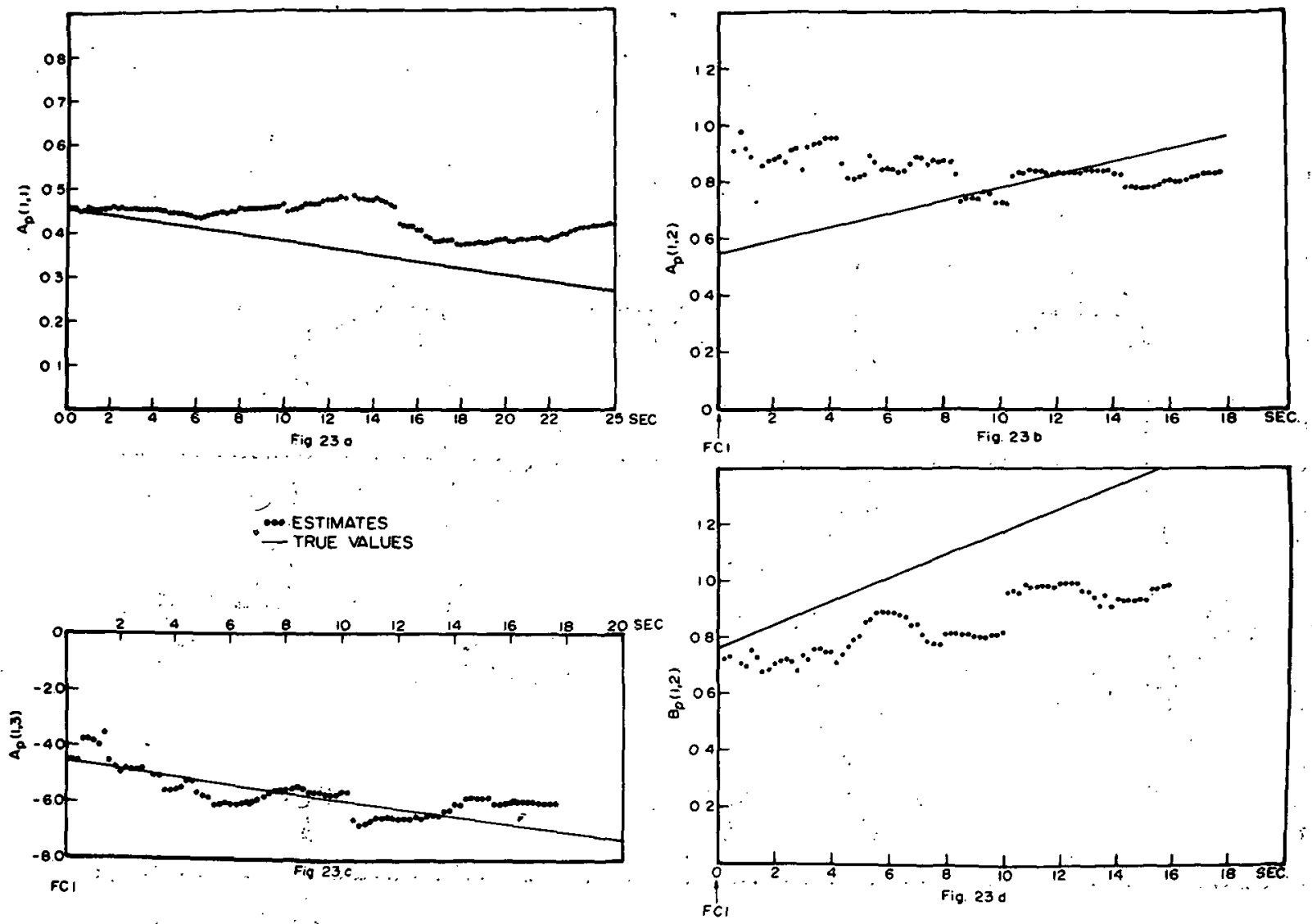

FIG 23 WEIGHTED LEAST SQUARES PARAMETER ESTIMATES-SINGLE STAGE ADAPTIVE CONTROL LOGIC 


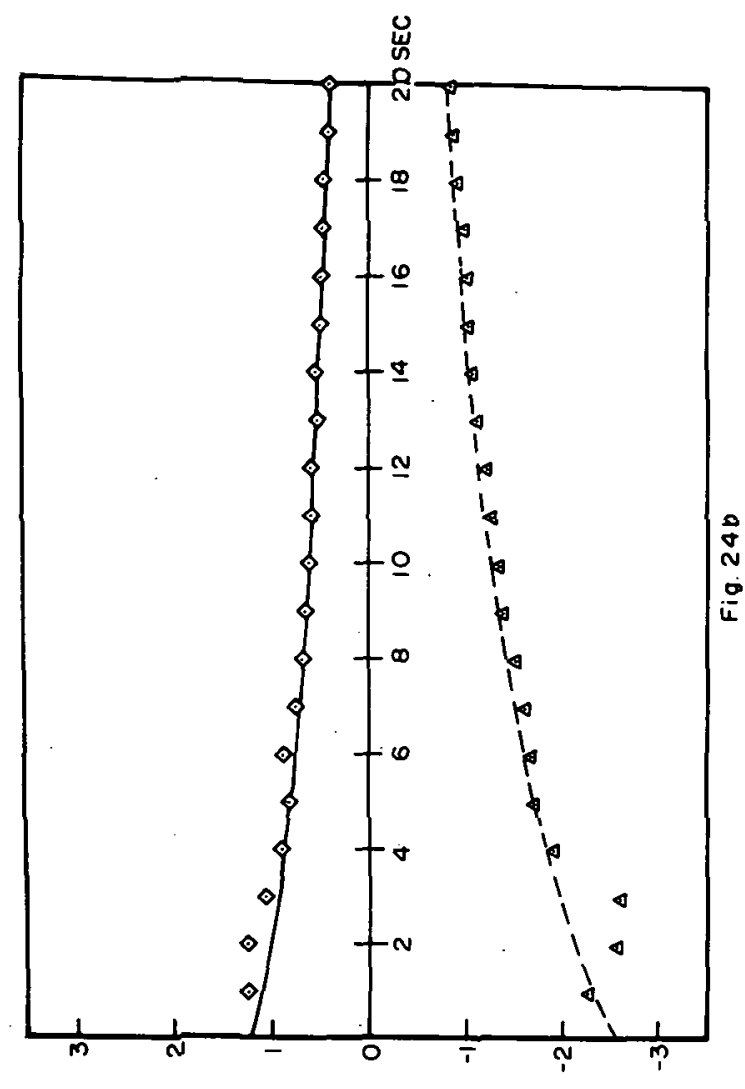

3184 Mọh woys xav8033د

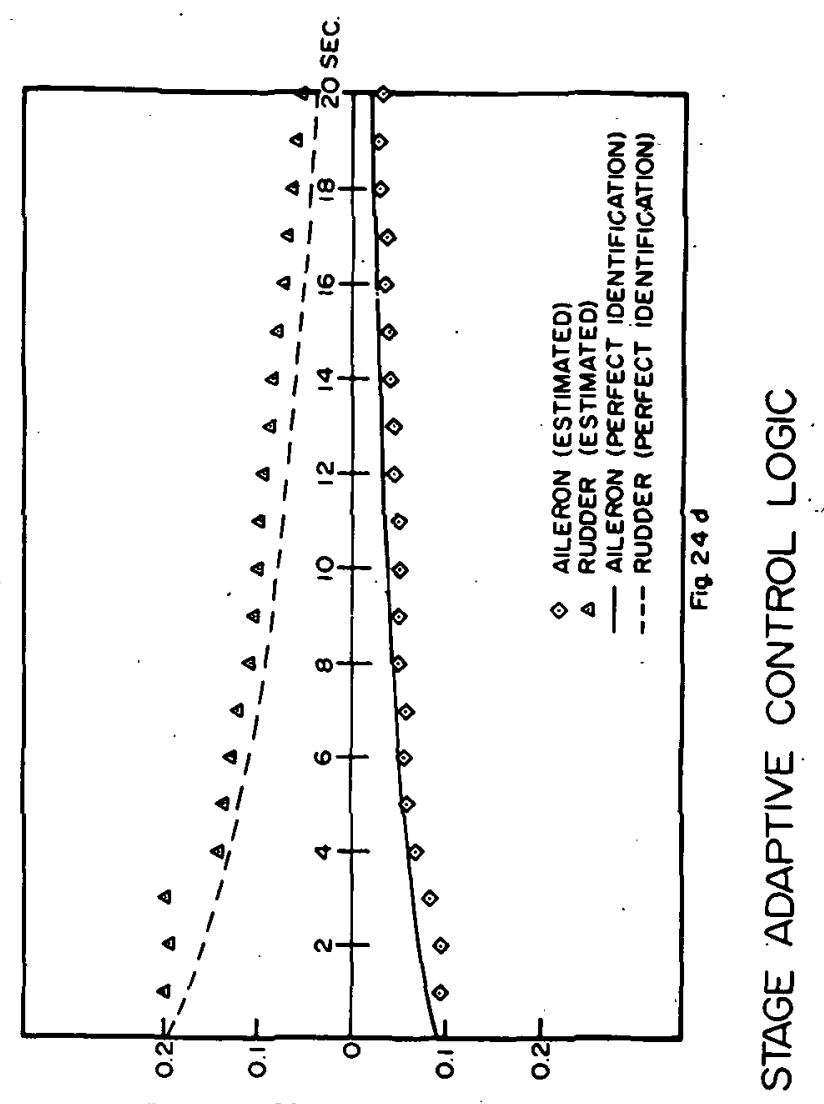

3.าอNจ רาoy woys *0\%8033t

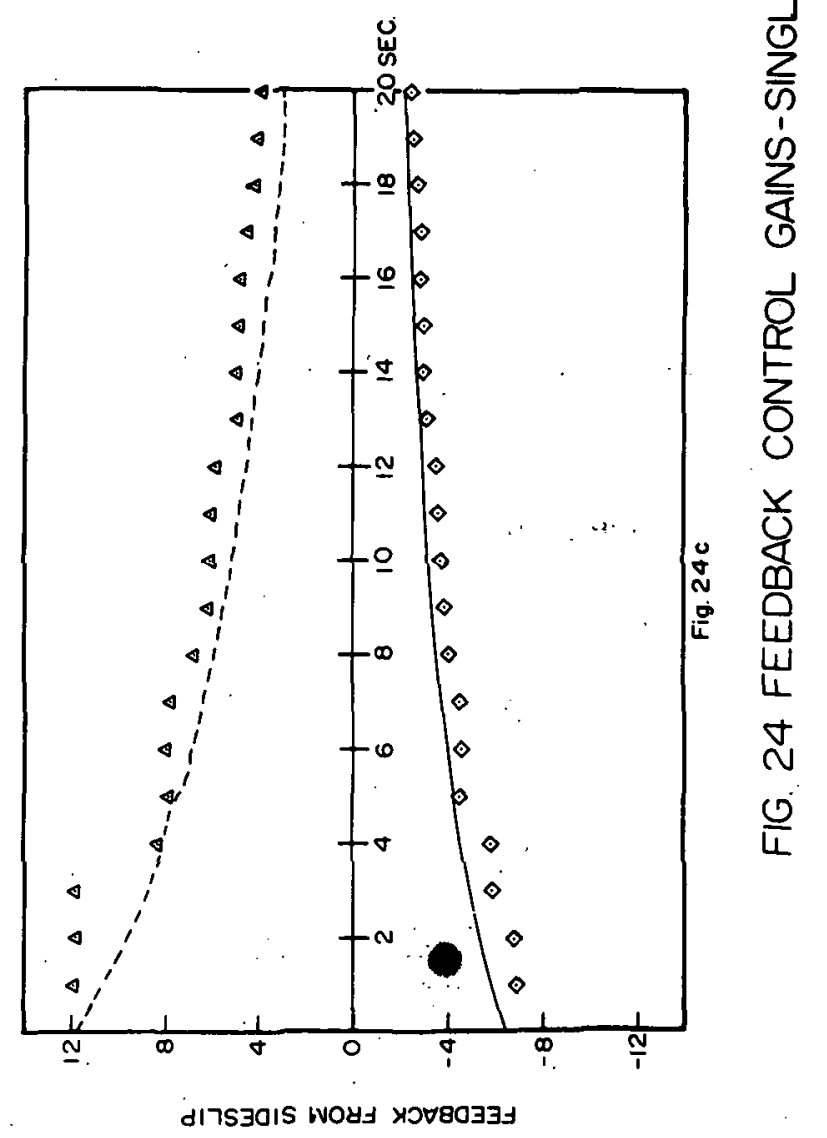




\subsubsection{Adaptive Control Loop Experiment - Linear Optimal Regulator Procedure}

objective: To study the behavior of the adaptive control system designed by coupling a weighted least squares identifier with the Riccati gain update logic as shown in fig. 12 .

Procedure: Using noisy state measurements with no prefiltering, parameter estimates were obtained at each control sample period and then used at each gain update sample in one iteration of the Riccati eq. (2.31). The gains were then computed from eq. 2.29, and along with the noisy state measurements used for control computation.

Design factors:

Pilot input: $\pm .5^{\circ}$ aileron, $.2 \mathrm{hz}$ square wave Control sample period: $0.1 \mathrm{sec}$. Gain adaptation periods: 1, $5 \mathrm{sec}$.

Parameters identified: $A_{p}(1,1), A_{p}(1,2), A_{p}(3,1), A_{p}(3,4)$, $B_{p}(1,1), B_{p}(1,2), B_{p}(2,2), B_{p}(3,1)$.

Remaining parameter: Set at the average value computed over the 6 given FC's.

Measurement noise: As defined in Table I. Results:

\section{$\underline{\text { Responses }}$}

Figure $25 a$ and $b$ depict roll rate and side slip responses for a gain update of 5.0 sec. These responses are very similar to those of fig. 21 (for noisy feedback but with perfect identification of the above 8 parameters) indicating 

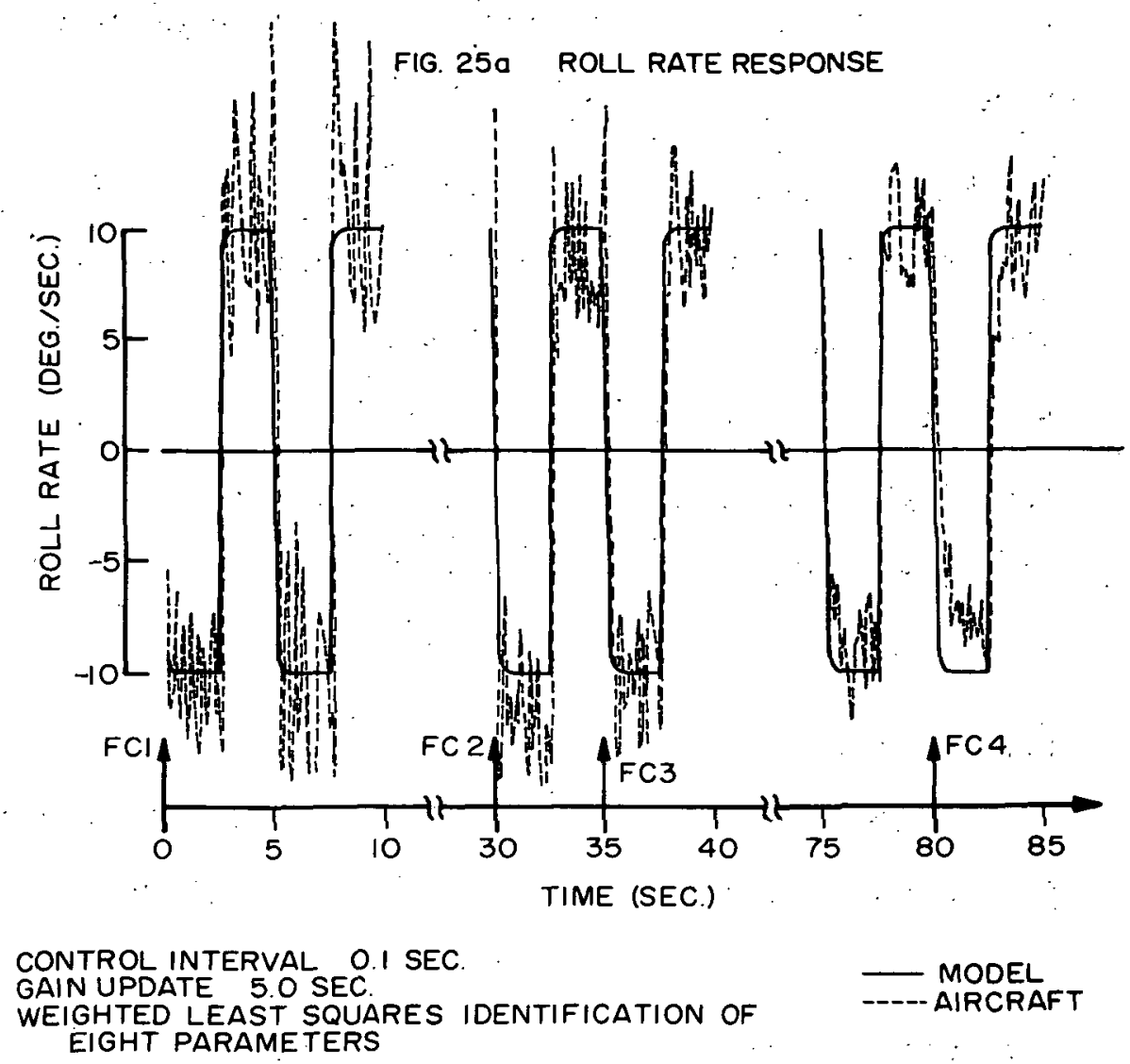

FIG. 25b SIDESLIP RESPONSE
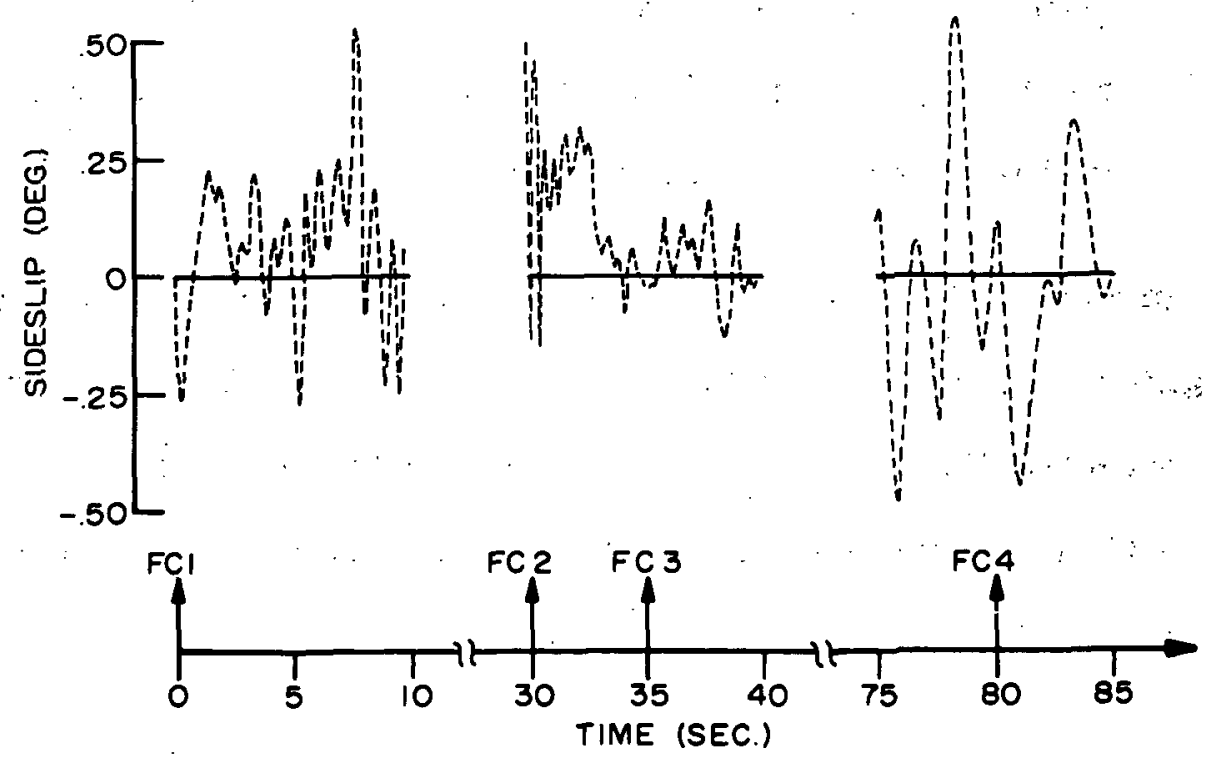

FIG. 25 LINEAR REGULATOR ADAPTIVE CONTROL LOGIC - 
that the inclusion of an actual identifier did not seriously degrade the performance. With a gain update period of 1.0 sec., improvements in the model following characteristics were noted.

Parameter tracking

Figures 26a, b and $\mathrm{c}$ illustrate typical parameter tracking. Again as in 4.5.1 the presence of bias in the estimate is readily evident as well as the capability for tracking the variation. As might be expected, a reduction of the rms values to $1 / 10$ those cited in Table I improved the estimates. Control gains

Comparisons between the gains computed based upon perfect identification of all parameters and the actual adapted gains are shown in figs. 27a, b, c, d, for part of the interval between FCl and FC4. Despite the differences between the adapted and optimal gains (most notably in the feedback gains affecting roll rate) the general variations in the gains were followed.

Conclusions: In view of the facts that the procedure was capable of adapting the feedback gains to reasonable values and the simularity between figs. 21 and 25, it is expected that the incorporation of prefiltering and an unbiased parameter estimation algorithm should produce an acceptable adaptive control system. 

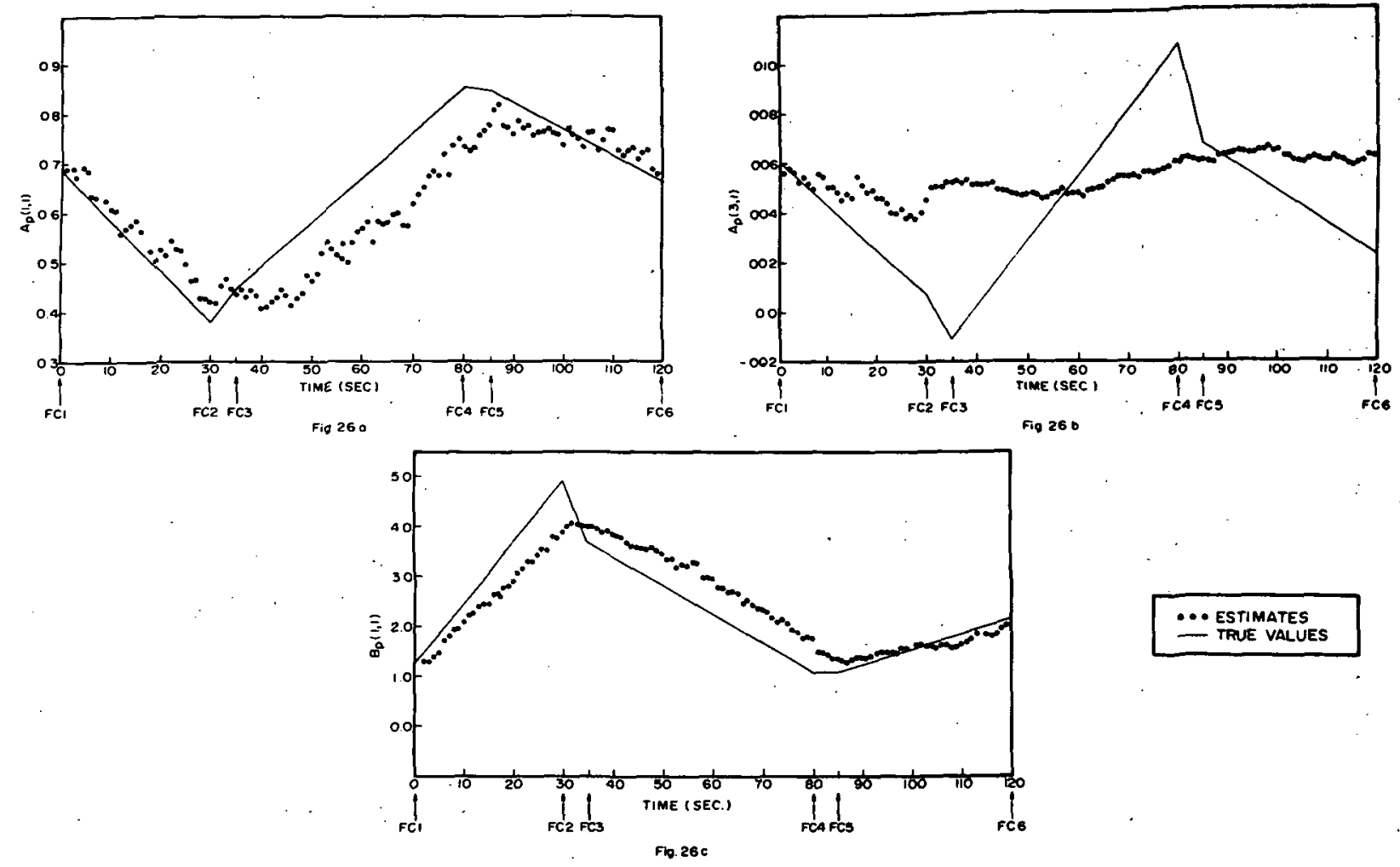

FIG. 26 WEIGHTED LEAST SQUARES PARAMETER ESTIMATES - LINEAR REGULATOR ADAPTIVE CONTROL LOGIC 

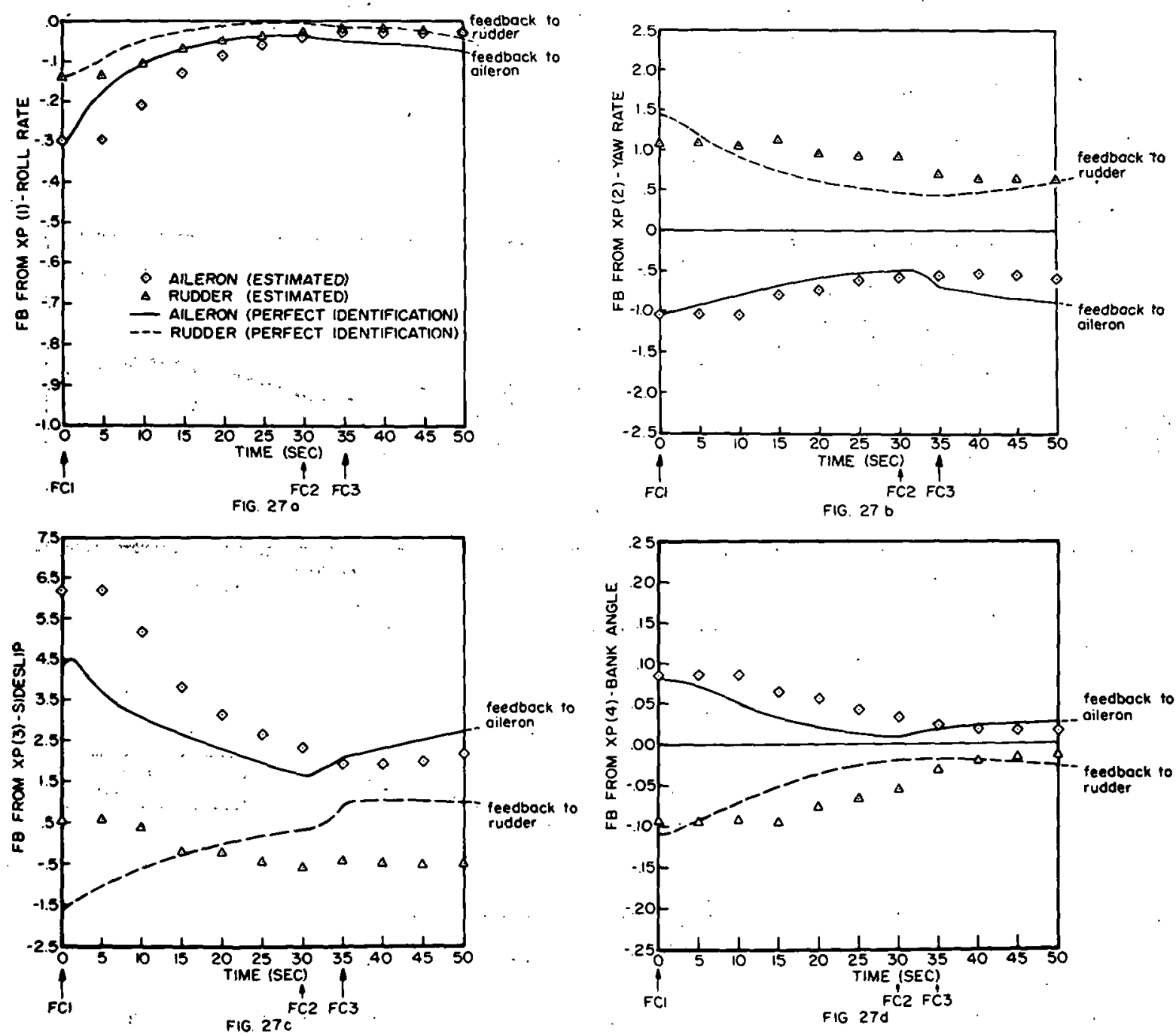

FIG. 27 FEEDBACK. CONTROL GAINS - LINEAR REGULATOR ADAPTIVE CONTROL LOGIC 


\subsection{Hybrid Computer Tests}

In addition to the previously described all digital simulations, the EAI 690 analog-digital hybrid computer at NASA LRC was used for system evaluation. The aircraft dynamics and pilot controls were programed on the analog portion of the computer, while the discrete control laws were pro:gramed on the digital portion.

\subsubsection{Linear Simulation Results}

The first tests were performed using the linearized aircraft.

dynamics. These tests had as their objectives:

Evaluation of feedback controllers in a hybrid environment.

Evaluation of the gain adaptation algorithm using perfect paremeter identification.

The results indicated that:

Noise in the analog to digital converters can be a significant problem for the nondynamic feedback controller developed using the single stage algorithm. The "type one" feedback controller developed using linear optimal control theory was much less sensitive to this noise. In particular, a noisy side slip measurement caused trouble for the single stage controller because of the moderately large gains necessary to effect good model following.

Even when no information about the actual flight condition was included in the initial Riccati matrices; only one or two executions of the gain update algorithm were necessary to produce gains giving accurate model-following. Figure 28a illustrates the aircraft response to a pilot aileron input using incorrect gains (those 
FIG. 28 FLIGHT CONDITION
FIG. 280
$M=.67$
$H=6100 \mathrm{M}$
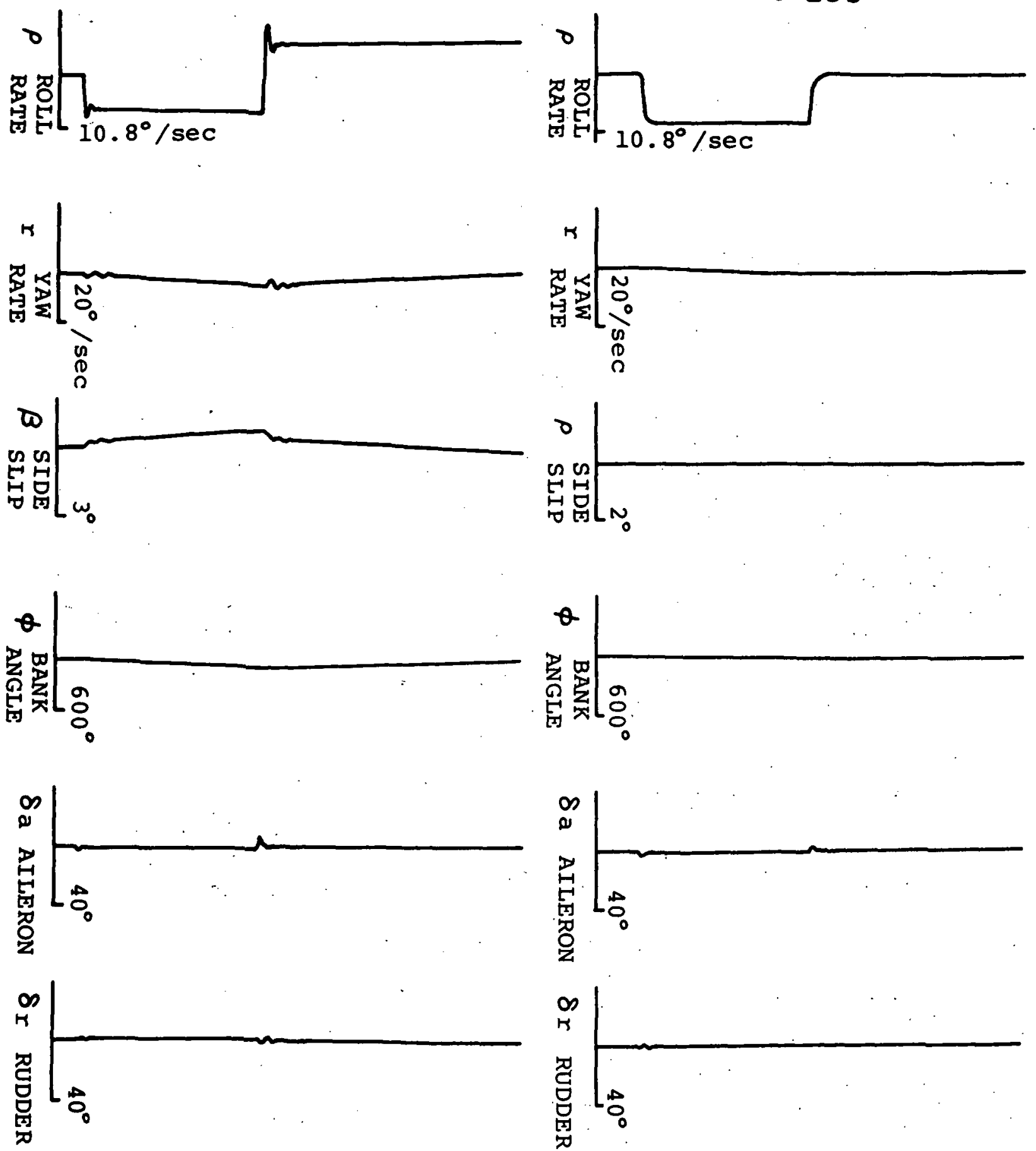

GAINS BASED ON

$$
\begin{aligned}
& M=.3 \\
& H=.0
\end{aligned}
$$


designed for FCl actually applied to the aircraft at FC6), while fig. $28 \mathrm{~b}$ illustrates the aircraft response after just one iteration of the gain update algorithm. This aircraft response is indistinguishable from the model response.

4.6.2 Nonlinear Simulation Results

A second set of preliminary tests were performed using a

nonlinear 6 degree-of-freedom aircraft analog simulation and the same dymamic feedback controller as described above. To avoid the logic needed to account for discontinuities in aircraft bank angle at $\varnothing=180^{\circ}$, the sine of this angle was fed back.

The lateral control system maintained the same performance for small perturbations about the trim condition as it had in the linear case, and did not fail to operate under extreme maneuvers. Figure 29 illustrates some of this behavior. 

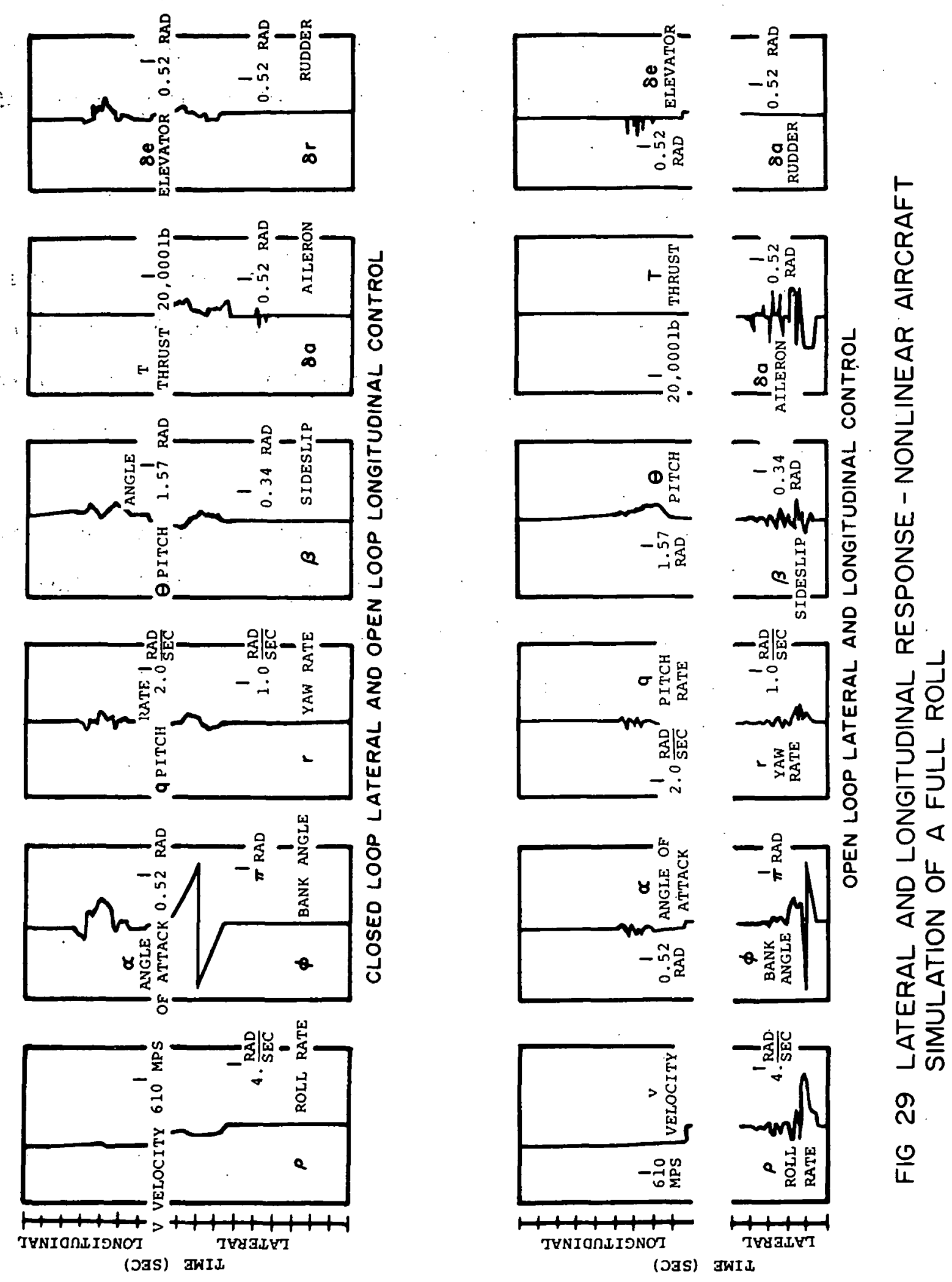


\subsection{DISCUSSION AND CONCLUUSIONS}

5.1. Results Discussion

In sumary, the results presented in section 4.0 lead to the following observations:

- Digital adaptive flight controllers can be designed using either linear-quadratic optimal regulator theory or single stage performance functions.

- Because of superior stability margins, the design based upon optimal regulator theory requires fewer parameters for identification. The performance of the overall adaptive control system fig. 25 is comparable to the performance of the "adaptive" controller with perfect identification simulated fig. 2l. This was based upon experiments using measurement noise with the characteristics of Table I.

- The adapted control gains in general track the variations in the optimal gains. However, some bias is evident especially in the feedback gains involving roll rate.

- Prefiltering of the noisy measurements prior to use in feedback control computation is needed.

- Usage of the noisy measurements themselves in the weighted least squares identification algorithms appears permissible. Identification of only parameters, as opposed to estimation of states and parameter together, appears preferable in view of the results obtained and the computational and linearization requirements of the latter. 
Application of the linearly designed controller to the actual six degree of freedom coupled system should yield acceptable results in view of the results discussed in section 4.6.2.

\subsection{Feasibility of Implementation}

Both of the adaptive control algorithms discussed in the preceding should be readily implementable in a digital flight computer. For purposes of illustration, consider an add time of three micro-seconds and a multiply time of six micro-seconds.

For the single stage design discussed in section 3.2 the following timing requirements would be typical for a fourth order system with two control inputs and with weighted least squares identification being performed for 12 parameters:

$\begin{array}{lr}\text { Control computation } & .378 \mathrm{~ms} \\ \text { Parameter identification } & 5.13 \mathrm{~ms} \\ \text { Gain computation } & 1.05 \mathrm{~ms}\end{array}$

For estimation using an extended Kalman filter the timing requirement would be $34.2 \mathrm{~ms}$. Thus if at the worst the above three functions were performed at each and every sample, a minimum sample period of about $7 \mathrm{~ms}$ would be needed for weighted least squares identification and about $40 \mathrm{~ms}$ if extended Kalman filtering were used.

Similarly for the optimal design described in section 3.3 , the following would be typical timing requir ements for a fourth order system with two controls and weighted least squares estimation of eight parameters (identification of 12 parameters was not necessary): Control computation $.414 \mathrm{~ms}$ 
Parameter identification

Gain computation
$1.54 \mathrm{~ms}$

$6.99 \mathrm{~ms}$

Thus if in the worst case these three computations had to be performed at each and every sample, a minimum period of about $10 \mathrm{~ms}$ would be required.

\subsection{Recommendations for future research}

In view of the experimental results discussed in section 4.0 , it is recommended that the following topics be studied:

- Design of filters to be used for smoothing the measurements prior to control computation.

- The use of prefiltered measurements for identification.

- A stability analysis of the single stage design perhaps utilizing the procedures followed by Chan ${ }^{10}$. These would incorporate the use of a perfect model following controller despite the non-satisfaction of the conditions of perfect model following.

- Applicability of the linear controller design to the $c$ nonlinear six-degree-of freedom system which incorporates coupling between lateral and longitudinal motion.

- Performance when true actuator dynamics, bending modes, and turbulence are considered.

- The need for dither for improving identification.

- The relative performance of an implicit adaptive controller based on hyperstability principles (see e.g., 'Landau, ref. 6). 


\section{References}

1. Kass, P. J., "Fly by Wire Advantages Explored," Aviation Week and Space Technology, July 10, 1972, pp. 52-54.

2. Sutherland, Major J., "Fly by Wire Control systems," AGARD Conference Proceedings No. 52, Advanced Control System Concepts, Sept. 1968, pp. $51-72$.

3. : Smyth, R. and Ehler's, H. L., "Survey of Adaptive Control Applications to Aerospace Vehicles," AGARD Conference Proceedings No. 52, Advanced Control System Concepts; Sept. 1968, pp. 3-13.

4. Ostgaard, M. A., "Case for Adaptive Control," AGARD Conference Proceedings No. 52, Advanced Control System Concepts, Sept. 1968, pp. $15-27$.

5. "Advanced Concepts for Guidance and Control of Tactical Missiles," TR-170-5, The Analytical Sciences Corporation, June 30, 1973.

6. Landau; I. D. and Courtiol, B., "Adaptive Model Following Systems for Flight Control and Simulation," AIAA Paper No: 72-95, AIAA I0th Aerospace Sciences Meeting, San Diego, California, Jan. 1972.

7. Asseo, S. J., "Application of Optimal Control to Perfect Model Following," JACC, June 1968.

8. Winsor, C: A., "Design of Model Reference Adaptive Control Systems by Liapunov's Second Method," IEEE Trans. on Automatic Control, April 1968.

9. Picon-Jimenez, J. and Montgomery, R. C., "Design of a Digital Adaptive Control System for Reentry Vehicles," 9th Allerton Conference on Circuit and Systems Theory, Monticello, IIl., 1971.

10. Chan, Y. T., "Perfect Model Following with a Real Model," 1973 JACC, pp. 287-293. 
11. Erzberger, H., "On the Use of Algebraic Methods in the Analysis and $\therefore$ Design of Model-Following Control systems," NASA TN D-4663, Ames Research Center, July 1968.

12. Motyka, P..R., "Variability stability Simulations Techniques for . Nonlinear Rate Dependent Systems," 1972 JACC, pp. 266-271.

13. Tiroshi, I. and Elliott, J., "A 'Type One' Explicit Model Following Adaptive Scheme", AIAA Guidance and Control Conference, Key Biscayne, Fla., 1973.

14. Porter, B., "Optimal Control of Multivariable Linear Systems Incorporating Integral Feedback," Electronics Letters, Vol. 7, No. 8, pp. $170-172$.

15. Anderson, B. D. O., and Moore, J. B., Linear Optimal Control, Prentice Hall, 1971.

16. Kreindler, E., "On the Iinear optimal Servo", Int. J. Control, Vol. 9, No. 4, 1969, pp. 465-472.

17. Larson, D., "Quasilinearization Techniques," Proc. of the 1969 National' Electronics Conference, Chicago, pp. 95-99.

18. Kaufman, H., "Aircraft Parameter Identification Using Kalman Filtering," Proc. of the 1969 National Electronics Conference, Chicago, pp. 85-89.

19. Fitzgerald, R. J., "Divergence of the Kalman Filter" IEEE, Automatic Control, Vol. AC-16, No. 6, Dec. 1971, pp. 736-747.

20. Young, P. C., "Applying Parameter Estimation to Dynamic Systems Part 1", Control Engineering, oct. 1969, Vol. 16, No. 10, 119-125.

21. Mendel, J. M., Discrete Techniques of Parameter Estimation, Marcel Dekker, Inc., New York, 1973. 
22. Saridis,G. N. and G. Stein, Stochastic Approximation Algorithms for Iinear Discrete-Time System Identification, IEEE Trans. on Automatic Control, AC-13, Oct. 1968, pp. 515-524.

23. Kaufman, H., "Kalman Filtering and Quasilinearization: A Comparative Discussion of Two Procedures For Parameter Estimation," IEEE, International Conference on Information Theory; Noordwijk, Netherlands, June 1970.

24. Graupe, D., "Identification of Systems," Von Nostrand Reinhold Co., 1972, pp. 229-230.

25: Sorenson, H. W., and Sacks, J. E., "Recursive Fading Memory Filtering," Information Sciences 3 (1971), 101-119.

26. Anderson, B. D: 0., "Experimental Data Weighting in Kalman-Bucy Filter," Information Sciences 5, 217-230 (1973). 
Appendix A

Aircraft Continuous Lateral Matrices

Corresponding to Equation 1.1.

$F$

FCl $\left[\begin{array}{c}-3 \\ -9\end{array}\right.$

FC2

$$
\left[\begin{array}{cccc}
-10.22 & -.1416 & -147.8 & 0 \\
.0671 & -.9610 & 29.43 & 0 \\
-.0101 & -.9958 & -.5613 & .0309 \\
.9997 & .0245 & 0 & 0
\end{array}\right]
$$

FC3

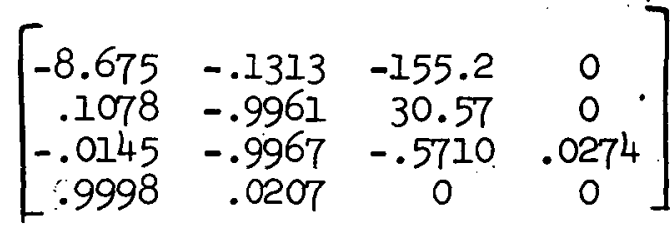

FC4

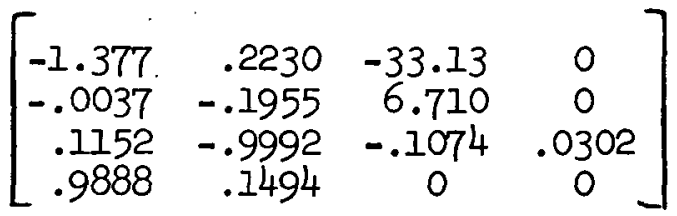

FC5

$$
\left[\begin{array}{cccc}
-1.525 & .0678 & -30.02 & 0 \\
-.0116 & -.1502 & 5.159 & 0 \\
.0698 & -.0992 & -.0903 & .0350 \\
.9945 & .1044 & 0 & 0
\end{array}\right]
$$

FC6

$$
\left[\begin{array}{cccc}
-4.033 & .0630 & -53.69 & 0 \\
-.0158 & -.3688 & 8.814 & 0 \\
.0236 & -.9971 & -.2333 & .0463 \\
.9983 & .0592 & 0 & 0
\end{array}\right]
$$

MODEI

$$
\left[\begin{array}{cccc}
-10.0 & 0 & -10.0 & 0 \\
0 & -.7 & 9 . & 0 \\
0 & -1 . & -.7 & 0 \\
1 & 0 & 0 & 0
\end{array}\right]
$$

G

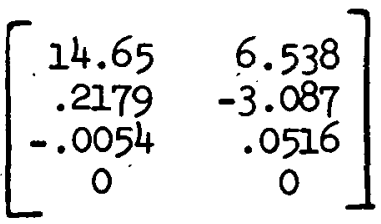

$\left[\begin{array}{cc}77.86 & 42.61 \\ .9165 & -14.40 \\ -.0247 & .0864 \\ 0 & 0\end{array}\right]$

$\left[\begin{array}{cc}54.30 & 43.22 \\ .7347 & -9.961 \\ -.0176 & .0538 \\ 0 & 0\end{array}\right]$

$\left[\begin{array}{cc}11.63 & 4.435 \\ .2086 & -1.761 \\ -.0014 & .0107 \\ 0 & 0\end{array}\right]$

$\left[\begin{array}{cc}11.51 & 5.241 \\ .1894 & -1.968 \\ -.0030 & .0135 \\ 0 & 0\end{array}\right]$

$\left[\begin{array}{cc}26.04 & 11.96 \\ .3293 & -4.823 \\ -.0100 & .0528 \\ 0 & 0\end{array}\right]$

$\left[\begin{array}{cc}20 & 2.8 \\ 0 & -3.13 \\ 0 & 0 \\ 0 & 0\end{array}\right]$ 
SS Lateral Controller Gain Matrices,

$Q=\operatorname{diag}(1,0,1,0), R=\operatorname{diag}(0,0)$, Sampling Period .2 sec.
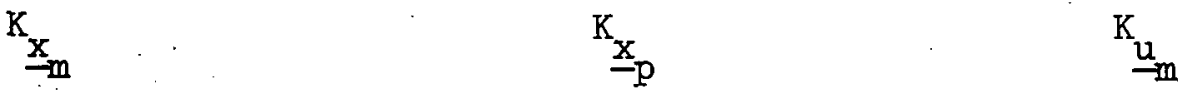

$p \quad r \quad \beta \quad \phi \quad r \quad{ }^{p} \quad \beta \quad \delta_{a} \delta_{r}$

FCl

$$
\left[\begin{array}{rrrr}
.070 & .911 & -4.15 & 0 . \\
-.112 & -2.34 & 10.3 & 0 .
\end{array}\right]\left[\begin{array}{rrrr}
-.178 & 1.22 & -6.61 & -.092 \\
.114 & -2.57 & 11.9 & .197
\end{array}\right]\left[\begin{array}{rr}
.890 & -.179 \\
-.144 & . .770
\end{array}\right]
$$

FC2

$$
\left[\begin{array}{rrrr}
.019 & .203 & -.929 & 0 . \\
.002 & -.645 & 2.83 & 0 .
\end{array}\right] \quad\left[\begin{array}{rrrr}
.021 & .347 & -.175 & -.012 \\
-.001 & -.535 & 1.62 & .018
\end{array}\right] \quad\left[\begin{array}{rr}
.245 & -.033 \\
.028 & .223
\end{array}\right]
$$

FC3

$$
\left[\begin{array}{rrrr}
.023 & . .517 & -2.32 & 0 . \\
.004 & -.925 & 4.05 & 0 .
\end{array}\right] \quad\left[\begin{array}{rrrr}
.039 & .681 & -3.14 & -.021 \\
-.004 & -.752 & 2.22 & .023
\end{array}\right] \quad\left[\begin{array}{rr}
.0297 & -.132 \\
.048 & .320
\end{array}\right]
$$

$\mathrm{FC4}$

$$
\left[\begin{array}{rrrr}
.080 & 1.52 & -6.85 & 0 . \\
.036 & -4.37 & 19.2 & 0 .
\end{array}\right]\left[\begin{array}{rrrr}
.241 & 1.97 & -10.2 & -.060 \\
.323 & -4.94 & 22.2 & .153
\end{array}\right]\left[\begin{array}{rr}
1.02 & -.368 \\
-.454 & 1.41
\end{array}\right]
$$

FC5 $\quad\left[\begin{array}{rrr}.076 & 1.63 & -7.33 \\ .017 & -3.84 & 16.9\end{array}\right.$

$\left.\begin{array}{l}0 . \\ 0 .\end{array}\right]\left[\begin{array}{rrrr}.277 & 2.09 & -10.9 & -.074 \\ .196 & -4.42 & 20.4 & .157\end{array}\right] \quad\left[\begin{array}{rrr}.970 & -.413 \\ -.217 & 1.27\end{array}\right]$

FC6

$$
\left[\begin{array}{rrr}
.038 & .611 & -2.76 \\
-.001 & -1.59 & 6.98
\end{array}\right.
$$

0$.

$\left[\begin{array}{rrrr}.108 & .845 & -4.62 & -.041 \\ .034 & -1.71 & 7.55 & .083\end{array}\right]$

$\left[\begin{array}{rr}.490 & -.136 \\ -.013 & .537\end{array}\right]$ 


\section{Appendix B}

Optimal Regulator Gain Matrices,

$Q=\operatorname{diag}\left(10^{-4}, 0,10^{4}, 0\right), R=\operatorname{diag}\left(10^{2}, 10^{2}\right)$,

Sampling Period .I sec.

FCI

$$
\begin{aligned}
& \begin{array}{llll}
p & r & \beta & \phi
\end{array} \\
& K_{21}=\left[\begin{array}{rrrc}
-.302 & -1.04 & 4.4 & .08 \\
-.145 & 1.42 & -1.7 & -.115
\end{array}\right] \\
& \text { Feedback from } x_{p} \\
& \begin{aligned}
\mathrm{K}_{22}= & {\left[\begin{array}{ll}
-1.54 & -.08 \\
-.30 & -1.28
\end{array}\right] \delta_{\mathrm{a}} } \\
& \text { Feedback from } \mathrm{u}_{\mathrm{p}}
\end{aligned} \\
& \begin{array}{l}
{\left[\begin{array}{ll}
-1.54 & -.08 \\
-.30 & -1.28
\end{array}\right] \delta_{a}} \\
\text { Feedback from } u_{p}
\end{array} \\
& \mathrm{~K}_{23}=\left[\begin{array}{cccc}
.0975 & .55 & -1.10 & 0.0 \\
.025 & -1.15 & 1.25 & 0.0
\end{array}\right] \\
& \text { Feedback from } x_{m} \\
& \text { Sa } 5 \\
& \mathrm{~K}_{24}=\left[\begin{array}{lr}
1.33 & -.23 \\
.13 & .95
\end{array}\right] \\
& \text { Feedback from } u_{m}
\end{aligned}
$$

\begin{tabular}{|c|c|c|c|c|c|c|}
\hline $\mathrm{K}_{21}=$ & {$\left[\begin{array}{l}-.025 \\
-.005\end{array}\right.$} & $\begin{array}{l}-.50 \\
+.47\end{array}$ & $\begin{array}{l}1.7 \\
0.3\end{array}$ & $\left.\begin{array}{r}.02 \\
-.02\end{array}\right]$ & $\mathrm{K}_{22}=$ & {$\left[\begin{array}{l}-1.36 \\
-.08\end{array}\right.$} \\
\hline$K_{23}=$ & {$\left[\begin{array}{l}.025 \\
.0075\end{array}\right.$} & $\begin{array}{r}.35 \\
-.70\end{array}$ & $\begin{array}{l}-1.0 \\
1.75\end{array}$ & $\left.\begin{array}{l}0.0 \\
0.0\end{array}\right]$ & $\mathrm{K}_{24}=$ & {$\left[\begin{array}{l}.33 \\
.08\end{array}\right.$} \\
\hline
\end{tabular}

\begin{tabular}{|c|c|c|c|c|c|c|c|}
\hline$K_{21}=$ & $E .040$ & $\begin{array}{r}-.67 \\
.45\end{array}$ & $\begin{array}{l}2.0 \\
0.8\end{array}$ & $\left.\begin{array}{r}.025 \\
-.015\end{array}\right]$ & $\mathrm{K}_{22}=$ & {$\left[\begin{array}{r}-1.34 \\
-.14\end{array}\right.$} & $\begin{array}{r}.30 \\
-1.3\end{array}$ \\
\hline $\mathrm{K}_{23}=$ & {$\left[\begin{array}{l}.0275 \\
.010\end{array}\right.$} & $\begin{array}{r}.53 \\
-.75\end{array}$ & $\begin{array}{l}-1.3 \\
1.65\end{array}$ & $\left.\begin{array}{l}0.0 \\
0.0\end{array}\right]$ & $\mathrm{K}_{24}=$ & {$\left[\begin{array}{l}.38 \\
.10\end{array}\right.$} & $\begin{array}{r}-.2 \\
.4\end{array}$ \\
\hline
\end{tabular}

FC2

FC3 
$\mathrm{FC} 4$

$\begin{aligned} & \mathrm{K}_{21}=\left[\begin{array}{llll}-.54 & -1.2 & 4.9 & .035 \\ -.23 & 1.6 & -.6 & -.05\end{array}\right] \\ & \mathrm{K}_{23}=\left[\begin{array}{cccc}.113 & .58 & -.95 & 0.0 \\ .0275 & -1.25 & 0.90 & 0.0\end{array}\right]\end{aligned} \mathrm{K}_{22}=\left[\begin{array}{ll}-1.63 & -.20 \\ -.37 & -1.06\end{array}\right]$

FC5

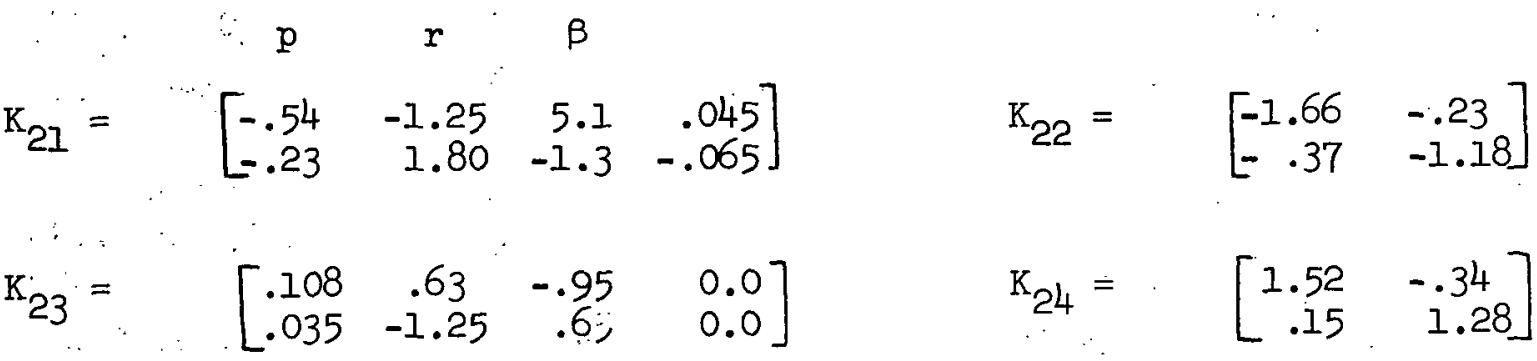

FC6

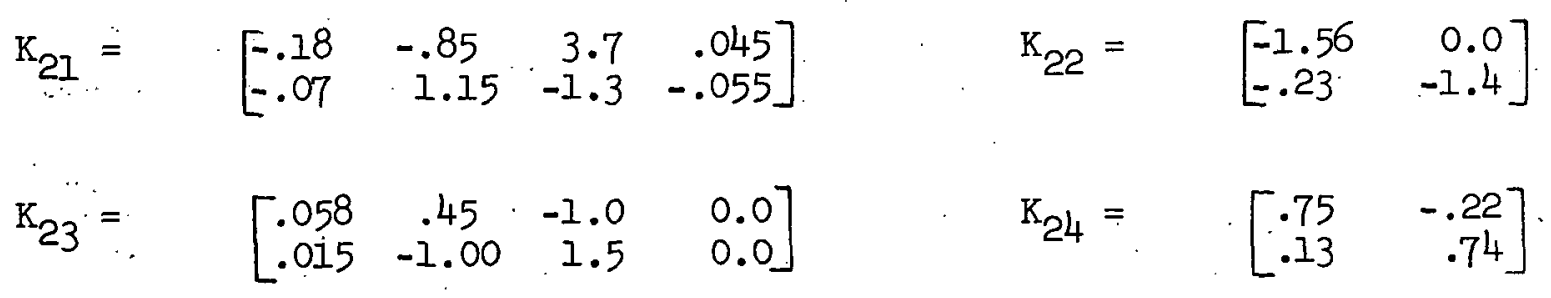

U. S. GOVERNMENT PRINTING OFFICE: 1974-640-046/44 
"The aeronautical and space activities of the United States shall be conducted so as to contribute ... to the expansion of buman knowledge of phenomena in the atmosphere and space. The Administration shall provide for the widest practicable and appropriate dissemination of information concerning its activities and the results thereof."

-National Aeronautics and SPACE ACt OF 1958

\section{NASA SCIENTIFIC AND TECHNICAL PUBLICATIONS}

TECHNICAL REPORTS: Scientific and technical information considered important, complete, and a lasting contribution to existing knowledge.

TECHNICAL NOTES: Information less broad in scope but nevertheless of importance as a contribution to existing knowledge.

TECHNICAL MEMORANDUMS:

Information receiving limited distribution because of preliminary data, security classification, or other reasons. Also includes conference proceedings with either limited or unlimited distribution.

CONTRACTOR REPORTS: Scientific and technical information generated under a NASA contract or grant and considered an important contribution to existing knowledge.
TECHNICAL TRANSLATIONS: Information published in a foreign language considered to merit NASA distribution in English.

SPECIAL PUBLICATIONS: Information derived from or of value to NASA activities. Publications include final reports of major projects, monographs, data compilations, handbooks, sourcebooks, and special bibliographies.

\section{TECHNOLOGY UTILIZATION}

PUBLICATIONS: Information on technology used by NASA that may be of particular interest in commercial and other non-aerospace applications. Publications include Tech Briefs, Technology Utilization Reports and Technology Surveys. 